\title{
Multi-Label Classification Models for the Prediction of Cross-Coupling Reaction Conditions
}

Michael Maser, Alexander Cui, Serim Ryou, Travis DeLano, Yisong Yue, Sarah Reisman

Submitted date: $14 / 10 / 2020$ - Posted date: 15/10/2020

Licence: CC BY-NC-ND 4.0

Citation information: Maser, Michael; Cui, Alexander; Ryou, Serim; DeLano, Travis; Yue, Yisong; Reisman, Sarah (2020): Multi-Label Classification Models for the Prediction of Cross-Coupling Reaction Conditions. ChemRxiv. Preprint. https://doi.org/10.26434/chemrxiv.13087769.v1

Machine-learned ranking models have been developed for the prediction of substrate-specific cross-coupling reaction conditions. Datasets of published reactions were curated for Suzuki, Negishi, and C-N couplings, as well as Pauson-Khand reactions. String, descriptor, and graph encodings were tested as input representations, and models were trained to predict the set of conditions used in a reaction as a binary vector. Unique reagent dictionaries categorized by expert-crafted reaction roles were constructed for each dataset, leading to context-aware predictions. We find that relational graph convolutional networks and gradient-boosting machines are very effective for this learning task, and we disclose a novel reaction-level graph-attention operation in the top-performing model.

File list (2) 


\title{
Multi-Label Classification Models for the Prediction of Cross-Coupling Reaction Conditions
}

\author{
Michael R. Maser, ${ }^{\dagger, \S}$ Alexander Y. Cui, ${ }^{\ddagger}, \S$ Serim Ryou, ${ }^{\mathbb{9}, \S}$ Travis J. DeLano, $^{\dagger}$ \\ Yisong Yue ${ }^{\ddagger}$ and Sarah E. Reisman ${ }^{*, \dagger}$ \\ $\dagger$ Division of Chemistry and Chemical Engineering, California Institute of Technology, \\ Pasadena, California, USA \\ $\ddagger$ Department of Computing and Mathematical Sciences, California Institute of Technology, \\ Pasadena, California, USA \\ \Computational Vision Lab, California Institute of Technology, Pasadena, California, USA \\ $\S$ Equal contribution. \\ E-mail: reisman@caltech.edu
}

\section{Abstract}

Machine-learned ranking models have been developed for the prediction of substrate-specific cross-coupling reaction conditions. Datasets of published reactions were curated for Suzuki, Negishi, and $\mathrm{C}-\mathrm{N}$ couplings, as well as Pauson-Khand reactions. String, descriptor, and graph encodings were tested as input representations, and models were trained to predict the set of conditions used in a reaction as a binary vector. Unique reagent dictionaries categorized by expert-crafted reaction roles were constructed for each dataset, leading to context-aware predictions. We find that relational graph convolutional networks and gradient-boosting machines are very effective for this learning task, and we disclose a novel reaction-level graph-attention operation in the top-performing model.

\section{Introduction}

A common roadblock encountered in organic synthesis occurs when canonical conditions for a given reaction type fail in complex molecule settings. 1 Optimizing these reactions frequently requires iterative experimentation that can slow progress, waste material, and add significant costs to research. ${ }^{2}$ This is especially prevalent in catalysis, where the substrate-specific nature of reported conditions is often deemed a major drawback, leading to the slow adoption of new methods. ${ }^{1 / 3}$ If, however, a transformation's structure-reactivity relationships (SRRs) were well-known or predictable, this roadblock could be avoided and new reactions could see much broader use in the field. ${ }^{4}$

Machine learning (ML) algorithms have demonstrated great promise as predictive tools for chemistry domain tasks. ${ }^{5}$ Strong approaches to molecular property prediction ${ }^{\underline{6}}[\sqrt{9}$ and generative design ${ }^{10} 13$ have been developed, particularly in the field of medicinal chemistry. 14 Some applications have emerged in organic synthesis, geared mainly towards predicting reaction products, $\frac{15 \mid 16}{16}$ yield,, $17+20$ and selectivity. $21+25$ Significant effort has also been invested in computer-aided synthesis planning (CASP) and the development of retrosynthetic design algorithms. $27-30$

To supplement these tools, initial attempts have been made to predict reaction conditions in the forward direction based on the substrates and products involved. 31 Thus far, studies have focused on global datasets with millions of data points of mixed reaction types. Advantages of this approach include ample training data and the ability to query any transformation with a 
single model. However, the sparse representation of individual reactions is a major drawback, in that reliable predictions can likely only be expected for the most common reactions and conditions within. This precludes the ability to distinguish subtle variations in substrate structures that lead to different condition requirements, which is critical for SRR modeling.

In recent years, it has become a goal of ours to develop predictive tools to overcome challenges in selecting substrate-specific reaction conditions. Towards this end, we recently reported a preliminary study of graph neural networks (GNNs) as multi-label classification (MLC) models for this task. ${ }^{32}$ We selected four high-value reaction types from the cross-coupling literature as testing grounds: Suzuki, C-N, and Negishi couplings, as well as Pauson-Khand reactions (PKRs) $\stackrel{33}{ }$ Modeling studies indicated relational graph convolutional networks (R-GCNs) ${ }^{34}$ as uniquely suited for our learning problem. We herein report the full scope of our studies, including improvements to the R-GCN architecture and an alternative tree-based learning approach using gradient-boosting machines (GBMs).

\section{Approach and Methods}

A schematic representation of the overall approach is included in Figure 1. We direct the reader to our initial report ${ }^{32}$ for additional procedural explanations. i]

\subsection{Data acquisition and pre- processing}

A summary of the datasets studied here is shown in Table 1. Each dataset was manually preprocessed using the following procedure:

1. Reaction data was exported from Reaxys (B) query results (Figure 1A). .33136

2. SMILES strings ${ }^{37}$ of coupling partners and major products were identified for each reaction entry (i.e., data point).

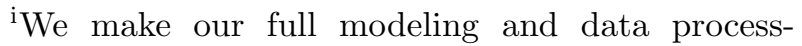
ing code freely available at https://github.com/ slryou41/reaction-gcnn.

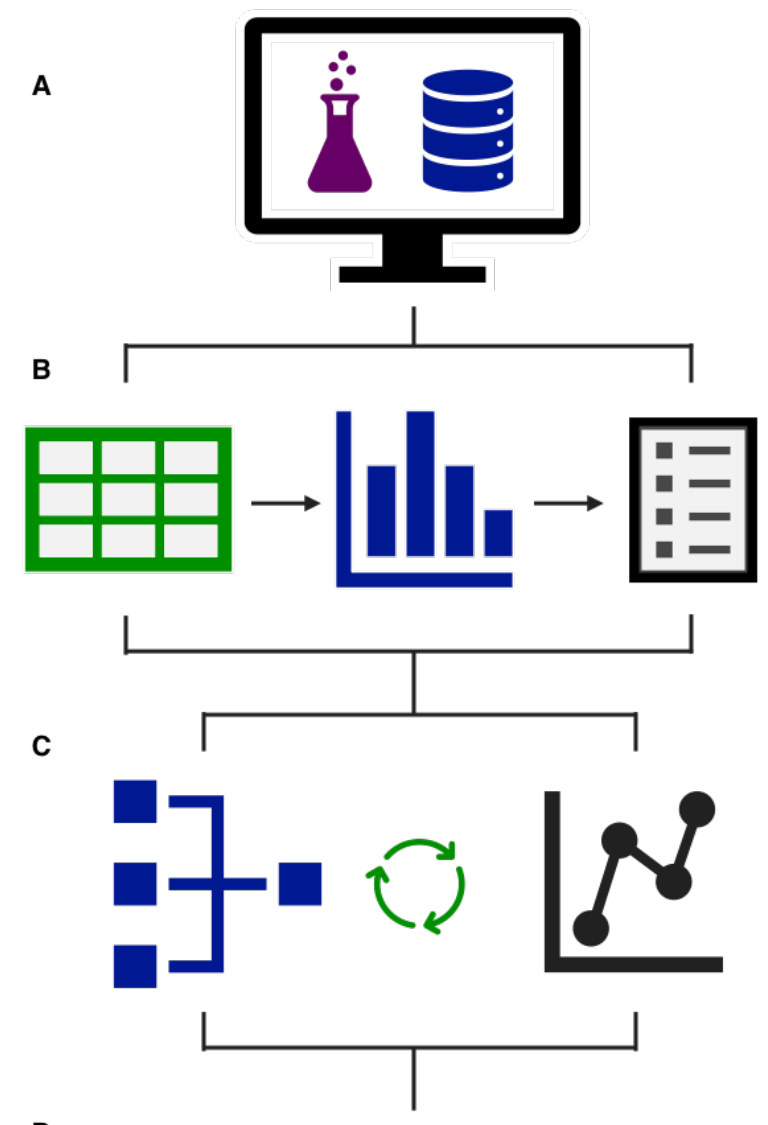

D

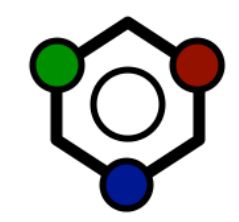

Figure 1: Schematic modeling workflow. A) Data gathering. B) Tabulation and dictionary construction. C) Iterative model optimization. D) Inference and interpretation.

3. Condition labels including reagents, catalysts, solvents, temperatures, etc. were extracted for each data point (Figure 1B).

4. All unique labels were enumerated into a dataset dictionary, which was sorted by reaction role and trimmed at a threshold frequency to avoid sparsity.

5. Labels were re-indexed within categories and applied to the raw data to construct binary condition vectors for each reaction. We refer to this process as binning.

The reactions studied here were chosen for their ubiquity and value in synthesis, breadth 
Table 1: Statistical summary of reaction datasets with Reaxys@ queries.

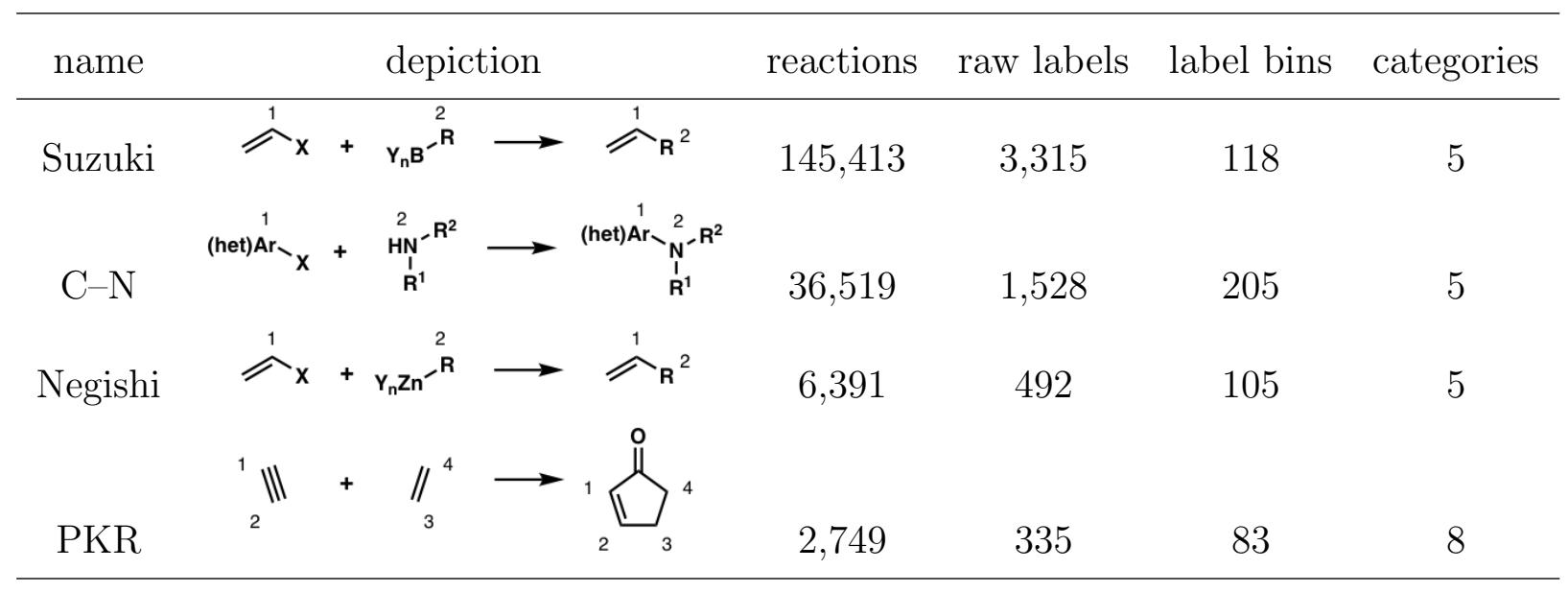

of known conditions, and range of dataset size and chemical space闬 It should be noted that certain parameters (e.g. temperature, pressure, etc.) were more fully recorded in some datasets than others. In cases where this data was wellrepresented, reactions with missing values were simply removed, or in the case of temperature and pressure were assumed to occur ambiently. However, when appropriate, these parameters were dropped from the prediction space to avoid discarding large portions of data.

The Suzuki dataset (Table 1, line 1) was obtained from a search of $\mathrm{C}-\mathrm{C}$ bond-forming reactions between $\mathrm{C}\left(\mathrm{sp}^{2}\right)$ halides or pseudohalides and organoboron species. Data processing returned $145 \mathrm{k}$ reactions with 118 label bins in 5 categories. Similarly, the $\mathrm{C}-\mathrm{N}$ coupling dataset (line 2) details reactions between aryl (pseudo)halides and amines, with $37 \mathrm{k}$ reactions and 205 bins in 5 categories. The Negishi dataset (line 3) contains $\mathrm{C}-\mathrm{C}$ bond-forming reactions between organozinc compounds and $\mathrm{C}\left(\mathrm{sp}^{2}\right)$ (pseudo)halides. After processing, this dataset gave $6.4 \mathrm{k}$ reactions with 105 bins in 5 categories. The PKR dataset (line 4) describes couplings of $\mathrm{C}-\mathrm{C}$ double bonds with $\mathrm{C}-\mathrm{C}$ triple bonds to form the corresponding cyclopentenones, containing $2.7 \mathrm{k}$ reactions with 83 bins in 8 categories. For all datasets, atom mapping was used as depicted in Table 1 to ensure only the desired transformation type was obtained iii Samples of the $\mathrm{C}-\mathrm{N}$ and Negishi label dictionaries are

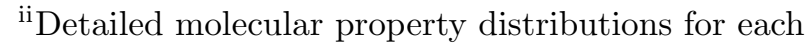

\begin{tabular}{|c|c|c|}
\hline \multicolumn{3}{|c|}{ C-N coupling dictionary sample } \\
\hline agent & label & category \\
\hline Cul & M1 & metal \\
\hline $\mathrm{Pd}_{2}(\mathrm{dba})_{3}$ & M2 & metal \\
\hline $\mathrm{Pd}(\mathrm{OAc})_{2}$ & M3 & metal \\
\hline- & - & - \\
\hline BINAP & L1 & ligand \\
\hline $\mathrm{P}(t-\mathrm{Bu})_{3}$ & L2 & ligand \\
\hline Xantphos & L3 & ligand \\
\hline- & - & - \\
\hline $\mathrm{NaOt}-\mathrm{Bu}$ & B1 & base \\
\hline $\mathrm{K}_{2} \mathrm{CO}_{3}$ & B2 & base \\
\hline $\mathrm{Cs}_{2} \mathrm{CO}_{3}$ & B3 & base \\
\hline- & - & - \\
\hline toluene & S1 & solvent \\
\hline 1,4-dioxane & $S 2$ & solvent \\
\hline DMF & S3 & solvent \\
\hline- & - & - \\
\hline 18-crown-6 & A1 & additive \\
\hline $\mathrm{Bu}_{4} \mathrm{NBr}$ & A2 & additive \\
\hline 8-quinolinol & A3 & additive \\
\hline
\end{tabular}

\begin{tabular}{|c|c|c|}
\hline \multicolumn{3}{|c|}{ Negishi coupling dictionary sample } \\
\hline agent & label & category \\
\hline $\mathrm{Pd}\left(\mathrm{PPh}_{3}\right)_{4}$ & M1 & metal \\
\hline $\mathrm{Pd}_{2}(\mathrm{dba})_{3}$ & M2 & metal \\
\hline $\mathrm{Pd}\left(\mathrm{PPh}_{3}\right)_{2} \mathrm{Cl}_{2}$ & M3 & metal \\
\hline- & - & - \\
\hline dppf & L1 & ligand \\
\hline Sphos & L2 & ligand \\
\hline Xphos & L3 & ligand \\
\hline- & - & - \\
\hline $\mathrm{LiCl}$ & A1 & additive \\
\hline $\mathrm{Zn}(0)$ & A2 & additive \\
\hline Cul & A3 & additive \\
\hline- & - & - \\
\hline THF & S1 & solvent \\
\hline DMF & S2 & solvent \\
\hline NMP & s3 & solvent \\
\hline- & - & - \\
\hline $\mathrm{T}<18$ & T1 & temp \\
\hline $18 \leq T<23$ & $\mathrm{~T} 2$ & temp \\
\hline $23 \leq \mathrm{T}<50$ & T3 & temp \\
\hline
\end{tabular}

Figure 2: Samples of categorized reaction dictionaries for C-N and Negishi datasets.

included in Figure 2, and full dictionaries for all reactions are provided in the SI.

\section{$2.2 \quad$ Model setup}

For each dataset, an 80/10/10 train/validation/test split was used in modeling. Training and test sets were kept consistent between model types for sake of comparability. Model inputs were prepared as reactant/product structure tuples, with encodings tailored to each learning method. Models were trained using binary

dataset can be found with our previous studies. 32

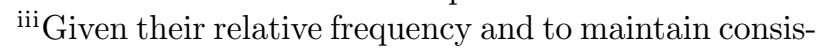
tent formatting, intramolecular couplings were dropped from the first three reactions but were retained for the PKR dataset. 


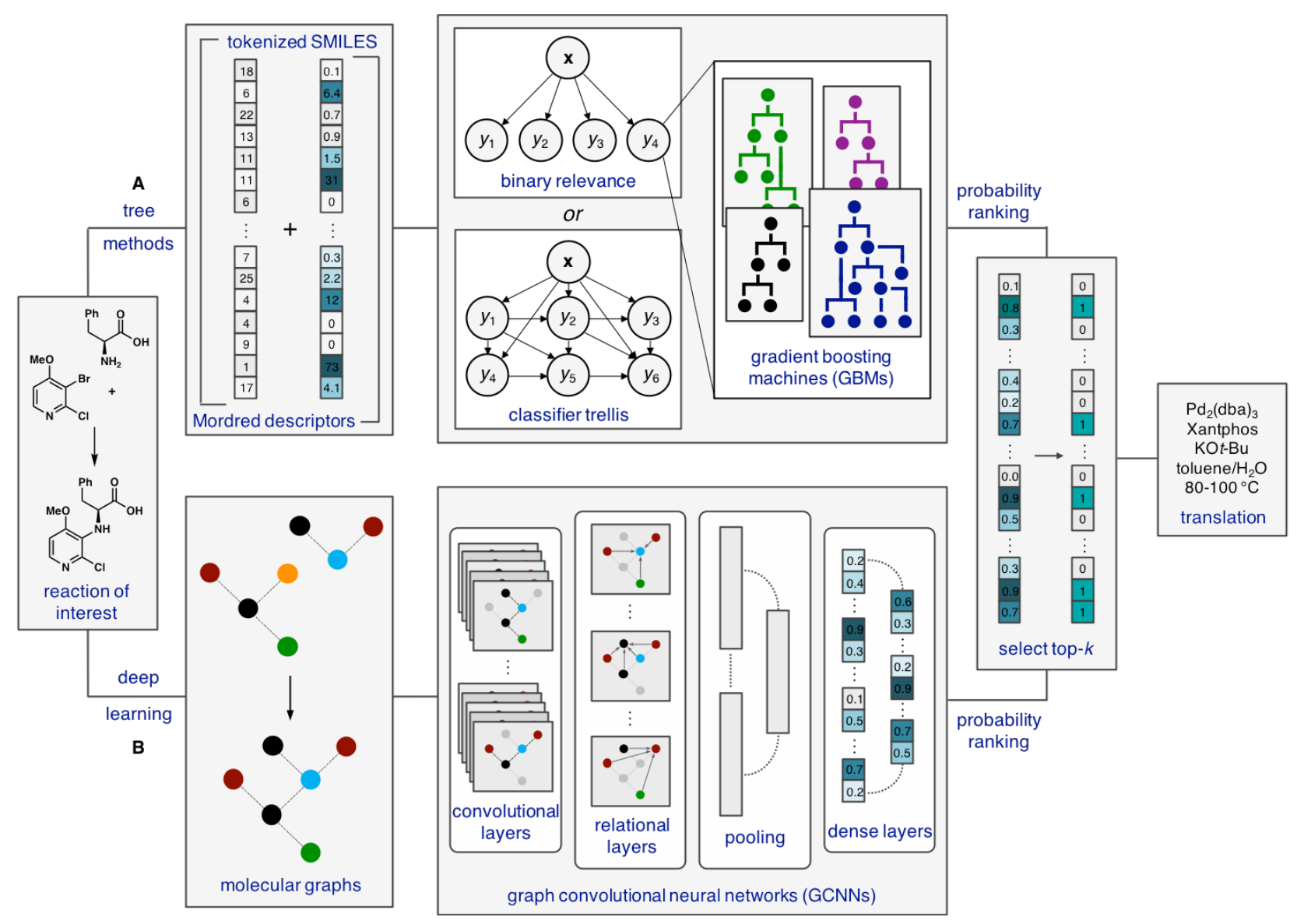

Figure 3: Schematic modeling workflow. A) Tree-based methods. String and descriptor vectors for each molecule in a reaction are concatenated and used as inputs to gradient-boosting machines (GBMs). B) Deep learning methods. Molecular graphs are constructed for each molecule in a reaction, which are passed as inputs to a graph convolutional neural network (GCNN). Both model types predict probability rankings for the full reaction dictionary, which are sorted by reaction role and translated to the final output.

cross-entropy loss to output probability scores for all reagent/condition labels in the reaction dictionary (Figure $1 \mathrm{C}$ ). The top- $k$ ranked labels in each dictionary category were selected as the final prediction, where $k$ is user-determined.

We define an accurate prediction as one where the ground-truth label appears in the top- $k$ predicted labels. Given the variable class-imbalance in each dictionary category, ${ }^{32138]}$ accuracy is evaluated at the categorical level as follows:

$$
A_{c}=\frac{1}{N} \sum_{i=1}^{N} \mathbb{1}\left[\hat{Y}_{i} \cap Y_{i}\right]
$$

where $\hat{Y}_{i}$ and $Y_{i}$ are the sets of top- $k$ predicted and ground truth labels for the $i$-th sample in category $c$, respectively. The correct instances are summed and divided by the number of samples in the test set, $N$, to give the overall test accuracy in the category, or $A_{c}$. 39

As a general measure of a model's performance, we calculate its average error reduction (AER) from a baseline predictor (dummy) that always predicts the top- $k$ most frequently occurring dataset labels in each category:

$$
\mathrm{AER}=\frac{1}{C} \sum_{c=1}^{C} \frac{A_{c}^{g}-A_{c}^{d}}{1-A_{c}^{d}},
$$

where $A_{c}^{g}$ and $A_{c}^{d}$ are the accuracies of the GNN and dummy model in the $c$-th category, respectively, and $C$ is the number of categories in the dataset dictionary. AER represents a model's average improvement over the naive approach 
that one might use as a starting point for experimental optimization. In other words, AER is the percent of the gap closed between the naive model and a perfect predictor of accuracy 1 .

\subsection{Model construction}

Both tree- and deep learning methods were explored for this MLC task (Figure 3), and their individual development is discussed below.

\subsubsection{Gradient-boosting machines}

GBMs are decision-tree-based learning algorithms that are popular in the ML literature for their performance in modeling numerical data. ${ }^{40}$ We explored several string and descriptor-based encodings as numerical inputs (see SI) and found that a hybrid encoding scheme provided the greatest learnability (Figure 3A) iv The hybrid inputs are a concatenation of tokenized SMILES strings for each molecule in a reaction (coupling partners and products), further concatenated with molecular property vectors obtained from the Mordred descriptor calculator. ${ }^{42}$ GBMs consistently outperformed other tree-based learners such as random forests (RFs), ${ }^{43}$ perhaps owing to their use of sequential ensembling to improve in poor-performance regions. 40

In our GBM experiments, a separate classifier was trained for all bins in a dataset dictionary, predicting whether or not they should be present in each reaction. Two general strategies have been developed for related MLC tasks, known as the binary relevance method (BM) and classifier chaining (CC). ${ }^{44}$ The BM approach considers each classifier as an independent model, predicting the label of its bin irrespective of the others. Conversely, CCs make predictions sequentially, taking the output of each label as an additional input for the next one, where the optimal order of chaining is a learned parameter. ${ }^{45}$ While the BM approach is significantly simpler from a computational perspective, CCs offer the potential for higher accuracy by modeling interdependencies between labels. 44

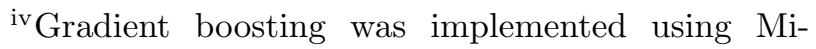
crosoft's LightGBM. $\underline{41}$
}

We saw modeling reagent correlations as prudent in our studies since they are frequently observed in synthesis. Some examples relevant to this work include using a polar protic solvent with an inorganic base, excluding exogenous ligand when using a pre-ligated metal source, setting the temperature below the boiling point of the solvent, etc. We decided to explore both methods, testing BM against a modern update to CCs introduced by Read and coworkers known as classifier trellises (CTs) ${ }^{46}$ In the CT method, instead of fully sequential propagation, models are fit in a pre-defined grid structure (the "trellis"), where the output of each prediction is passed to multiple downstream classifiers at once (Figure $3 \mathrm{~A}$, center). This eliminates the cost of chain structure discovery, while still benefiting from nesting predictions. 44

The ordering of a CT is enforced algorithmically starting from a seed label, chosen randomly or by expert intervention. From Read et al., the trellis is populated by maximizing the mutual information (MI) between source and target labels $\left(s_{\ell}\right)$ at each step $(\ell)$ as follows:

$$
s_{\ell}=\operatorname{argmax}_{k \in S} \sum_{j \in \mathrm{pa}(\ell)} I\left(y_{j} ; y_{k}\right),
$$

where $S$ and $\mathrm{pa}(\ell)$ are the set of remaining labels and the available trellis structure at the current step, respectively, and $y_{j}$ and $y_{k}$ are the $j$-th and $k$-th target labels, respectively. Here, $I\left(y_{j} ; y_{k}\right)$ represents the MI between labels $j$ and $k$ based on their co-occurrences in the dataset. The matrix of all pairwise label dependencies $I\left(Y_{j} ; Y_{k}\right)$ is constructed as below:

$$
I\left(Y_{j} ; Y_{k}\right)=\sum_{y_{j} \in \mathcal{Y}_{j}} \sum_{y_{k} \in \mathcal{Y}_{k}} p\left(y_{j}, y_{k}\right) \log \left(\frac{p\left(y_{j}, y_{k}\right)}{p\left(y_{j}\right) p\left(y_{k}\right)}\right),
$$

where $p\left(y_{j}, y_{k}\right)$, and $p\left(y_{j}\right)$ and $p\left(y_{k}\right)$ are the joint and marginal probability mass functions of $y_{j}$ and $y_{k}$, respectively. $\mathcal{Y}_{j}$ and $\mathcal{Y}_{k}$ represent the possible values $y_{j}$ and $y_{k}$ can each assume, which for our task of binary classification are both $\{0,1\}$. Full MI matrices and optimized trellises for each dataset are included in the SI, and an example is discussed with the results. 


\subsubsection{Relational graph convolutional networks}

Originally reported by Schlichtkrull et al., ${ }^{34} \mathrm{R}$ GCNs are a subclass of message passing neural networks (MPNNs) ${ }^{477}$ that explicitly model relational data such as molecular graphs. This is achieved by constructing sets of relation operations, where each relation $r \in \mathcal{R}$ is specific to a type and direction of edge between connected nodes. In our setting, the relations operate on atom-bond-atom triples using a learned, sparse weight matrix $\mathbf{W}_{r}^{(l)}$ in each layer $l{ }^{34} \mathrm{In}$ a propagation step, each current node representation $h_{i}^{(l)}$ is transformed with all relation-specific neighboring nodes $h_{j}^{(l)}$ and summed over all relations such that:

$$
h_{i}^{(l+1)}=\sigma\left(\sum_{r \in \mathcal{R}} \sum_{j \in \mathcal{N}_{i}^{r}} \frac{1}{c_{i, r}} \mathbf{W}_{r}^{(l)} h_{j}^{(l)}+\mathbf{W}_{0}^{(l)} h_{i}^{(l)}\right),
$$

where $\mathcal{N}_{i}^{r}$ is the set of applicable neighbors and $\sigma$ is an element-wise non-linearity, for us the tanh. The self-relation term $\mathbf{W}_{0}^{(l)} h_{i}^{(l)}$ is added to preserve local node information, and $c_{i, r}$ is a normalization constant. ${ }^{34}$ Unlike traditional GCNs, R-GCNs intuitively model edge-based messages in local sub-graph transformations. $\frac{34}{\text { This is }}$ potentially very powerful for reaction learning in that information on edge types (i.e., single, double, triple, aromatic, and cyclic bonds) is crucial for modeling reactivity.

Here, we extend the R-GCN architecture with an additional graph attention layer (GAL) at the final readout step inspired by graph attention networks (GATs) from Veličkovic ${ }^{48}$ and Busbridge. ${ }^{49}$ As described by Veličković et al. ${ }^{48}$ GALs compute pair-wise node attention coefficients $\alpha_{i j}$ for each node $h_{i}$ in a graph and its neighbors $h_{j}$. Two nodes' features are first transformed via a shared weight matrix $\mathbf{W}$, the results of which are concatenated before applying a learned weight vector and softmax normalization. The final update rule is simply a linear combination of $\alpha_{i j}$ with the newly transformed node vectors $\left(\mathbf{W} h_{j}\right)$, summed over all neighboring nodes and averaged over a set of parallel attention mechanisms. $\frac{48}{4}$

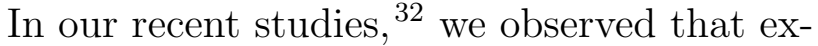
isting relational GATs (R-GATs) ${ }^{49}$ using atomlevel attention layers were less effective for our task than simple R-GCNs.] Inspired nonetheless by the chemical intuition of graph attention, we adapted existing GALs to construct a reaction-level attention mechanism. Instead of pair-wise $\alpha_{i j}$, we construct self-attention coefficients $\alpha_{i}^{m}$ for all nodes $h_{i}^{m}$ in a molecular graph $\boldsymbol{h}^{m}=\left\{h_{0}^{m}, h_{1}^{m}, \ldots, h_{L}^{m}\right\}$. As in GATs, we take a linear combination of $\alpha_{i}^{m}$ for all $L$ nodes in $\boldsymbol{h}^{m}$ after further transformation by matrix $\mathbf{W}^{g}$ :

$$
\begin{gathered}
\alpha_{i}^{m}=\sigma\left(\mathbf{W}^{s} h_{i}^{m}\right), \forall i \in\{1,2, \ldots, L\}, \\
h_{i}^{a}=\alpha_{i}^{m} \mathbf{W}^{g} h_{i}^{m},
\end{gathered}
$$

where $\mathbf{W}^{s}$ is the learned attention weight matrix, $\sigma$ is the sigmoid activation function, and $h_{i}^{a}$ is the updated node representation. The convolved graphs $\boldsymbol{h}^{a}=\left\{h_{0}^{a}, h_{1}^{a}, \ldots, h_{L}^{a}\right\}$ for each molecule $m$ are then concatenated on the node feature axis to give an overall reaction representation $\boldsymbol{h}^{r}$ that we term the attended reaction graph (ARG):

$$
\mathrm{ARG}=\boldsymbol{h}^{r}=\left[\|_{m=1}^{M} \boldsymbol{h}_{m^{a}}\right],
$$

where $M$ is the number of molecules in the reaction (reactants and products) and $\|$ denotes concatenation. Similar to the attention mechanism above, reaction-level attention coefficients $\alpha_{i}^{r}$ are then constructed and linearly combined with the ARG nodes $h_{i}^{r}$ after transformation with $\mathbf{W}^{v}$. The final readout vector $\boldsymbol{v}_{r}$ is obtained from the attention layer by summative pooling over the nodes:

$$
\begin{gathered}
\alpha_{i}^{r}=\sigma\left(\mathbf{W}^{r} h_{i}^{r}\right), \forall i \in\{1,2, \ldots, H\}, \\
\boldsymbol{v}_{r}=\sum_{i=1}^{H} \alpha_{i}^{r} \mathbf{W}^{v} h_{i}^{r},
\end{gathered}
$$

where $H$ is the total number of nodes and $\mathbf{W}^{r}$ is the reaction attention weight matrix. This con-

${ }^{\mathrm{v}}$ We found it necessary to reduce the hidden dimension of R-GATs to avoid excessive memory requirements relative to other GCNs, $\underline{48}$ and thus do not make a direct comparison of their performance. 
Table 2: Prediction accuracy for all model types on the Suzuki dataset.

\begin{tabular}{c|c|c|ccccc}
\hline dataset & top- $k$ & category & dummy & BM-GBM & CT-GBM & R-GCN & AR-GCN \\
\hline \multirow{4}{*}{ Suzuki } & AER & - & $-0.0263^{a}$ & $-0.0554^{b}$ & 0.2767 & $\mathbf{0 . 3 1 1 5}$ \\
\cline { 3 - 7 } & \multirow{4}{*}{ top-1 } & metal & 0.3777 & 0.5732 & 0.5629 & 0.6306 & $\mathbf{0 . 6 4 9 9}$ \\
& & ligand & 0.8722 & 0.8390 & 0.8408 & 0.9036 & $\mathbf{0 . 9 0 8 1}$ \\
& base & 0.3361 & 0.4908 & 0.4777 & 0.5455 & $\mathbf{0 . 5 8 9 6}$ \\
& & solvent & 0.6377 & 0.6729 & 0.6751 & 0.7049 & $\mathbf{0 . 7 2 1 7}$ \\
& & additive & 0.9511 & 0.9259 & 0.9196 & $\mathbf{0 . 9 6 2 4}$ & 0.9621 \\
\cline { 3 - 8 } & \multirow{4}{*}{ top-3 } & AER & - & 0.4088 & 0.3774 & 0.4936 & $\mathbf{0 . 5 2 4 6}$ \\
\cline { 3 - 7 } & & metal & 0.6744 & 0.8516 & 0.8475 & 0.8482 & $\mathbf{0 . 8 5 9 7}$ \\
& ligand & 0.9269 & 0.9635 & 0.9606 & 0.9644 & $\mathbf{0 . 9 6 7 6}$ \\
& & base & 0.7344 & $\mathbf{0 . 8 3 3 8}$ & 0.8250 & 0.8123 & 0.8285 \\
& solvent & 0.8013 & 0.8637 & 0.8577 & 0.8836 & $\mathbf{0 . 8 8 9 7}$ \\
& & additive & 0.9771 & 0.9842 & 0.9832 & $\mathbf{0 . 9 9 3 4}$ & 0.9931 \\
\hline
\end{tabular}

${ }^{a}$ AER excluding additive: 0.0962. ${ }^{b}$ AER excluding additive: 0.0922 .

struction differs from standard R-GCNs, which output readout vectors for individual molecules and concatenate them to form the ultimate reaction representation. Altogether, we term our hybrid architecture as an attended relational graph convolutional network, or AR-GCN.

In all deep learning experiments, with or without attention, the reaction vector readouts were passed to a multi-layer perceptron (MLP) of depth $=2$. vi The final prediction is made as a single output vector with one entry for each label in the reaction dictionary, and the result is translated as described in Section 2.2.

\section{Results and discussion}

\subsection{Model performance}

Our modeling pipeline was first tested on the Suzuki coupling dataset, the largest of the four. Table 2 summarizes top-1 and top-3 categorical accuracies (Equation 1) and AERs (Equation 2) for the following models: GBMs with no trellising (BM-GBM), GBMs with trellising (CT-GBM), standard R-GCNs as reported by Schlichtkrull et al. (R-GCN), $\underline{32134}$ our ARGCNs developed here (AR-GCN), and the dummy predictor as a baseline (dummy).

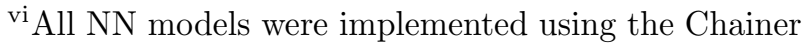
Chemistry (ChainerChem) deep learning library. 50
}

For this dataset, GCN models significantly outperformed GBMs across categories for both top-1 and top-3 predictions. While GBMs actually gave negative top-1 AERs over baseline, these scores were dominated by the additive contribution; excluding this category the BMand CT-GBMs gave modest $10 \%$ and $9 \%$ AERs, respectively. Despite struggling with top-1 predictions, GBMs gave significant AERs for top-3, with BM-GBMs at $41 \%$ and CT-GBMs at $38 \%$. The AR-GCNs gave the best accuracy of all models, providing $31 \%$ and $52 \%$ top- 1 and top-3 AERs, respectively. AR-GCNs gave roughly $3 \%$ AER gain over the R-GCN in both top- 1 and top-3 predictions, demonstrating the value of the added attention layer.

A few interesting categorical trends can be seen across model types. For instance, models provide the best error reduction $\left(\mathrm{ER}=\frac{A_{c}^{g}-A_{c}^{d}}{1-A_{c}^{d}}\right.$, see Equation 2) in the metal category, with the AR-GCN at $44 \%$ and $57 \%$ for top- 1 and top-3, respectively. Similarly, models perform well in the base category, where the AR-GCN gave the best top-1 ER and BM-GBMs gave the best top-3 ER. Less consistent ERs between top- 1 and top-3 predictions were obtained for the remaining three categories. For example, with solvents, the AR-GCN improved baseline by $23 \%$ in top- 1 predictions, but $44 \%$ in top- 3 . Likewise, for AR-GCN ligand predictions, a $28 \%$ ER was obtained for top-1 versus a $56 \%$ gain 
Table 3: Prediction accuracy for all model types on the C-N, Negishi, and PKR datasets.

\begin{tabular}{|c|c|c|c|c|c|c|c|}
\hline dataset & top- $k$ & category & dummy & BM-GBM & CT-GBM & R-GCN & AR-GCN \\
\hline \multirow{12}{*}{$\mathrm{C}-\mathrm{N}$} & \multirow{6}{*}{ top-1 } & AER & - & $-0.0413^{a}$ & $-0.1593^{b}$ & 0.3453 & 0.3604 \\
\hline & & metal & 0.2452 & 0.4825 & 0.4582 & 0.5989 & 0.6162 \\
\hline & & ligand & 0.5219 & 0.5538 & 0.5710 & 0.6981 & 0.7068 \\
\hline & & base & 0.2479 & 0.5028 & 0.5003 & 0.5932 & 0.6066 \\
\hline & & solvent & 0.3219 & 0.4582 & 0.4524 & 0.5647 & 0.5674 \\
\hline & & additive & 0.8904 & 0.7669 & 0.7031 & 0.8984 & 0.8997 \\
\hline & \multirow{6}{*}{ top-3 } & AER & - & 0.3568 & 0.3131 & 0.5391 & 0.5471 \\
\hline & & metal & 0.6526 & 0.7928 & 0.7772 & 0.8479 & 0.8490 \\
\hline & & ligand & 0.6647 & 0.7933 & 0.7928 & 0.8605 & 0.8688 \\
\hline & & base & 0.6400 & 0.8008 & 0.7916 & 0.8452 & 0.8370 \\
\hline & & solvent & 0.5677 & 0.7370 & 0.7281 & 0.7973 & 0.7997 \\
\hline & & additive & 0.9156 & 0.9290 & 0.9184 & 0.9534 & 0.9559 \\
\hline \multirow{12}{*}{ Negishi } & \multirow{6}{*}{ top-1 } & AER & - & 0.3510 & 0.2773 & 0.4439 & 0.4565 \\
\hline & & metal & 0.2887 & 0.5444 & 0.5218 & 0.6555 & 0.6730 \\
\hline & & ligand & 0.7879 & 0.8174 & 0.7900 & 0.8724 & 0.8772 \\
\hline & & temperature & 0.3317 & 0.6656 & 0.6527 & 0.6188 & 0.6507 \\
\hline & & solvent & 0.6938 & 0.8562 & 0.8514 & 0.8868 & 0.8915 \\
\hline & & additive & 0.8309 & 0.8691 & 0.8401 & 0.8724 & 0.8644 \\
\hline & \multirow{6}{*}{ top-3 } & AER & - & 0.5947 & 0.5199 & 0.6590 & 0.6833 \\
\hline & & metal & 0.5008 & 0.7771 & 0.7674 & 0.8086 & 0.8517 \\
\hline & & ligand & 0.8549 & 0.9548 & 0.9321 & 0.9522 & 0.9553 \\
\hline & & temperature & 0.5885 & 0.9031 & 0.8772 & 0.8517 & 0.8708 \\
\hline & & solvent & 0.8788 & 0.9321 & 0.9402 & 0.9537 & 0.9537 \\
\hline & & additive & 0.9043 & 0.9548 & 0.9354 & 0.9761 & 0.9729 \\
\hline \multirow{18}{*}{ PKR } & \multirow{9}{*}{ top-1 } & AER & - & 0.4396 & 0.4010 & 0.3973 & 0.4199 \\
\hline & & metal & 0.4302 & 0.7901 & 0.7786 & 0.7132 & 0.7057 \\
\hline & & ligand & 0.8792 & 0.9351 & 0.9237 & 0.9057 & 0.9094 \\
\hline & & temperature & 0.2830 & 0.5954 & 0.5649 & 0.6528 & 0.6642 \\
\hline & & solvent & 0.3321 & 0.6183 & 0.6260 & 0.6792 & 0.6981 \\
\hline & & activator & 0.6906 & 0.8244 & 0.8015 & 0.8415 & 0.8491 \\
\hline & & $\mathrm{CO}(\mathrm{g})$ & 0.7245 & 0.8855 & 0.8855 & 0.8717 & 0.8868 \\
\hline & & additive & 0.9057 & 0.9008 & 0.8893 & 0.8906 & 0.8491 \\
\hline & & pressure & 0.6528 & 0.8588 & 0.8702 & 0.8491 & 0.8491 \\
\hline & \multirow{9}{*}{ top-3 } & $\mathbf{A E R}^{c}$ & - & 0.6987 & 0.6740 & 0.6844 & 0.7145 \\
\hline & & metal & 0.7132 & 0.9351 & 0.9313 & 0.9057 & 0.8906 \\
\hline & & ligand & 0.9019 & 0.9962 & 0.9924 & 0.9849 & 0.9962 \\
\hline & & temperature & 0.5962 & 0.8740 & 0.8321 & 0.8528 & 0.8604 \\
\hline & & solvent & 0.5925 & 0.8779 & 0.8550 & 0.8679 & 0.8981 \\
\hline & & activator & 0.8830 & 0.9466 & 0.9275 & 0.9774 & 0.9774 \\
\hline & & $\mathrm{CO}(\mathrm{g})$ & 1.0000 & 1.0000 & 1.0000 & 1.0000 & 1.0000 \\
\hline & & additive & 0.9321 & 0.9885 & 0.9885 & 0.9698 & 0.9736 \\
\hline & & pressure & 0.9623 & 0.9771 & 0.9847 & 0.9849 & 0.9849 \\
\hline
\end{tabular}

${ }^{a}$ AER excluding additive: 0.2302. ${ }^{b}$ AER excluding additive: $0.2282 .{ }^{c}$ Excludes $C O(g)$. 

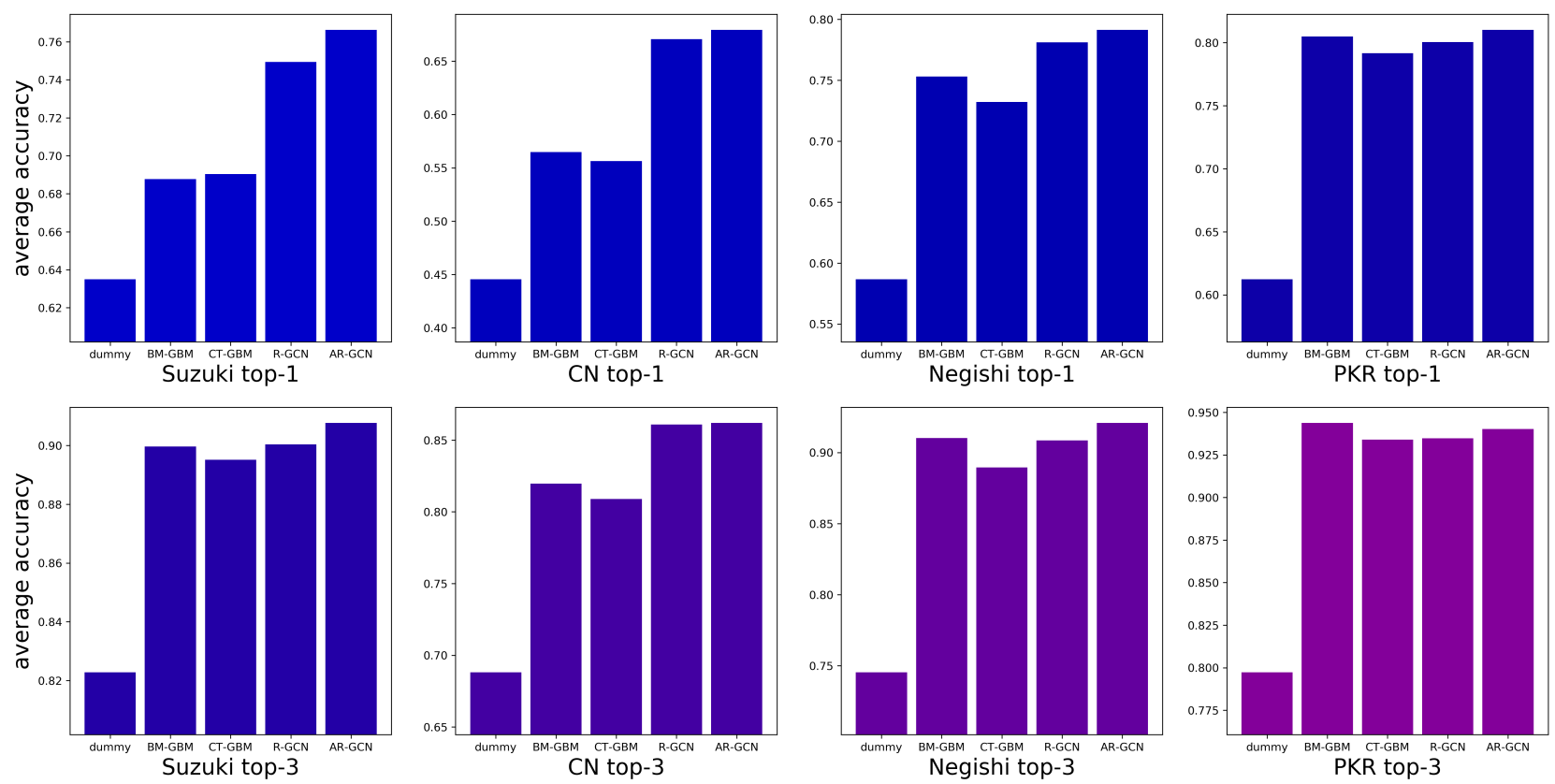

Figure 4: Average top-1 and top-3 categorical accuracies for each model across the four datasets.

in top-3. Finally, although the baseline additive accuracy is high as the majority of reactions are null in this category, the AR-GCN still gave a $23 \%$ top- 1 ER and a $70 \%$ top-3 ER.

The trends and differences between top-1 and top-3 performance gains are reflective of the frequency distributions in each label category. 32 These intuitively resemble long-tail or Paretotype distributions, $\stackrel{51}{ }$ with the bulk of the cumulative density contained in a small number of bins and the remaining bins supporting smaller frequencies. The distribution shapes are likely to influence the relative top-1 and top-3 AERs, where the highly skewed distributions could be more difficult to improve over baseline.

Having demonstrated the utility of our predictive framework, we turned to the remaining datasets to assess its scope. Modeling results for $\mathrm{C}-\mathrm{N}$, Negishi, and PKRs are detailed in Table 3 and Figure 4. Notable observations for each dataset are discussed below.

$C-N$ coupling. Similar to the Suzuki results, the AR-GCN was the top performer for $\mathrm{C}-\mathrm{N}$ couplings in almost all categories, and slightly higher AERs were observed overall. The ARGCN afforded $36 \%$ and $55 \%$ top-1 and top3 AERs, respectively, again providing slight gains over R-GCNs at $35 \%$ and $54 \%$. As above, GBMs struggled with this relatively large dataset (36,519 reactions) due to difficulties with the additive category. Models again made strong improvements in the metal and base categories, but also gave consistently strong gains for ligands and solvents, especially for top-3 predictions. For example, the AR-GCN returned top-3 ERs of $57 \%$ for metals, $61 \%$ for ligands, $55 \%$ for bases, and $54 \%$ for solvents. Note that these ERs correspond to very high accuracies $\left(A_{c}\right)$ of $85 \%, 87 \%, 84 \%$, and $80 \%$, respectively.

Negishi coupling. The highest AERs of all modeling experiments came with the Negishi dataset. The AR-GCN again gave the strongest performance, with top- 1 and top-3 AERs of $46 \%$ and $68 \%$, respectively. However, the R-GCN and even GBM models gave the highest accuracies in some categories. Interestingly, BM- and CT-GBMs performed significantly better than the GCNs for temperature predictions, though the strongest ER for most models came from the solvent category.

$P K R$. For the PKR dataset - the smallest of the four - simple BM-GBMs gave the best top-1 AER at $44 \%$, followed closely by the AR-GCN at $42 \%$. Similarly for top-3 predictions, these models gave AERs of $70 \%$ and $71 \%$, respectively. Compared to the other reactions, GCNs are perhaps more prone to overfitting this small of a dataset, ${ }^{[52}$ making tree-based modeling more 


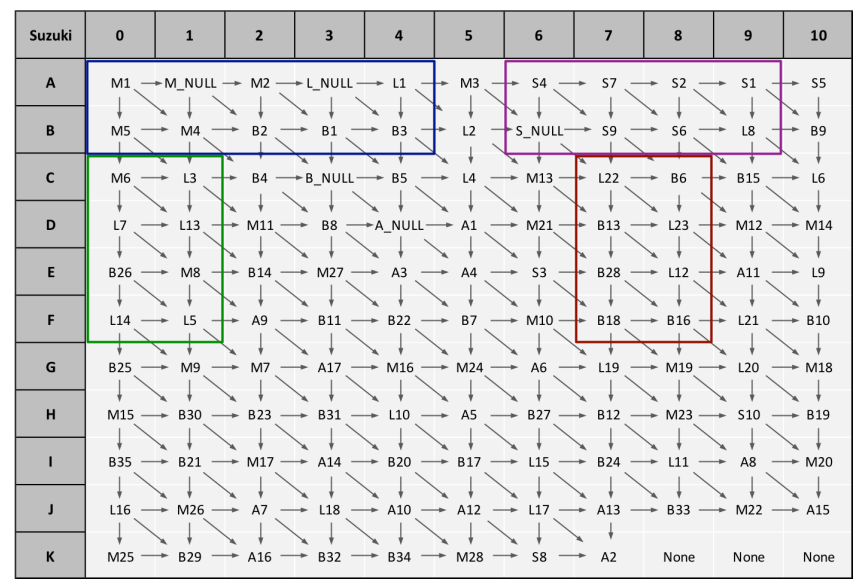

Figure 5: Optimized prediction trellis for the Suzuki dataset.

suitable. It is interesting to note that in general for PKRs, the GCN models were better at predicting physical parameters like temperature, solvent, and $C O(g)$ atmosphere, whereas GBMs gave better performance for reaction components such as metal, ligand, and additive.

\section{$3.2 \quad$ Interpretability}

\subsubsection{Tree methods}

Given the results described above, we sought an understanding of the chemical features informing our predictions. Tree-based learning is often favored in this regard in that feature importances (FIs) can be directly extracted from models. We found that FIs for our GBMs were roughly uniform across the SMILES regions of the encodings. The most informative physical descriptors from the Mordred vectors pertained to two classes: topological charge distributions ${ }^{53}$ correlated with local molecular dipoles; and Moreau-Broto autocorrelations ${ }^{\sqrt{54}}$ weighted by polarizability, ionization potential, and valence electrons (see SI for detailed rankings). The latter class is particularly intriguing as they are calculated from molecular graphs in what have been described as atom-pair convolutions, ${ }^{55}$ not unlike the GCN models used here. 34

An advantage to using CTs is the ability to extract their MI matrices and trellis structures

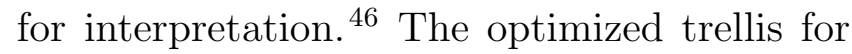
the Suzuki CT-GBMs is included in Figure 5. where several chemically intuitive features and category blocks can be noted:

1. Block A0-B4 (blue): The result of M1 $\left(\mathrm{Pd}\left(\mathrm{PPh}_{3}\right)_{4}\right)$ is used to predict three more metals: M2 $\left(\mathrm{Pd}(\mathrm{OAc})_{2}\right), \mathrm{M} 4\left(\mathrm{Pd}(\mathrm{dppf}) \mathrm{Cl}_{2}\right.$. $\mathrm{DCM})$, and M5 $\left(\mathrm{Pd}\left(\mathrm{PPh}_{3}\right)_{2} \mathrm{Cl}_{2}\right)$. Based on these metal complexes, the probability of using exogenous ligand (L_NULL) and L1 $\left(\mathrm{PPh}_{3}\right)$ is then predicted.

2. Block $\mathrm{C} 0-\mathrm{F} 2$ (green): The use of unligated M6 $\left(\mathrm{Pd}_{2}(\mathrm{dba})_{3}\right)$ informs the predictions of ligands L3 (XPhos), L7 ([(t-Bu) $\left.\left.{ }_{3} \mathrm{PH}\right] \mathrm{BF}_{4}\right)$, and $\mathrm{L} 13$ ( $\left.{ }^{\mathrm{Me}} \mathrm{CgPPh}\right)$. These in turn feed the model of unligated M8 $\left(\mathrm{Pd}(\mathrm{dba})_{2}\right)$, which then informs L5 $\left(\mathrm{P}(o \text {-tolyl })_{3}\right)$.

3. Block A6-B9 (purple): Several solvents are connected, where the predictions of S4 (1,4-dioxane) and $\mathrm{S} 7$ (PhMe) propagate through $\mathrm{S} 9\left(\mathrm{H}_{2} \mathrm{O}\right)$, S2 $(\mathrm{EtOH})$, and $\mathrm{S} 6$ $(\mathrm{MeCN})$. These additionally feed classifiers of S1 (THF) and S_NULL (neat).

4. Block C7-F8 (red): Four different classes of base are interwoven, including $\mathrm{B} 6(\mathrm{CsF})$ and $\mathrm{B} 13(\mathrm{KO} t-\mathrm{Bu})$. This informs the prediction of $\mathrm{B} 28\left(\mathrm{LiOH} \cdot \mathrm{H}_{2} \mathrm{O}\right)$, which then goes on to feed models of B18 (DIPEA) and $\mathrm{B} 16(\mathrm{NaO} t-\mathrm{Bu})$.

As a control experiment vii we withheld the propagated predictions from the CT-GBMs to test whether the MI was actually being used. $\frac{56}{}$ Indeed, model accuracy dropped off markedly, even below baseline in some categories. While this suggests that CT-GBMs do learn reagent correlations, the sharp performance loss may also indicate overfitting to this information. 46 Further studies are necessary to uncover the optimal molecule featurization in combination with CTs, though the results here suggest their promise in modeling structured reaction data.

\subsubsection{Deep learning methods}

For AR-GCNs, a valuable interpretability feature lies in the learned feature weights $\alpha_{i}^{r}$ (Equation 9). Intuitively, the weights represent the

\footnotetext{
${ }^{v i i}$ Detailed adversarial control studies for all GBM models are included in the SI. 56
} 


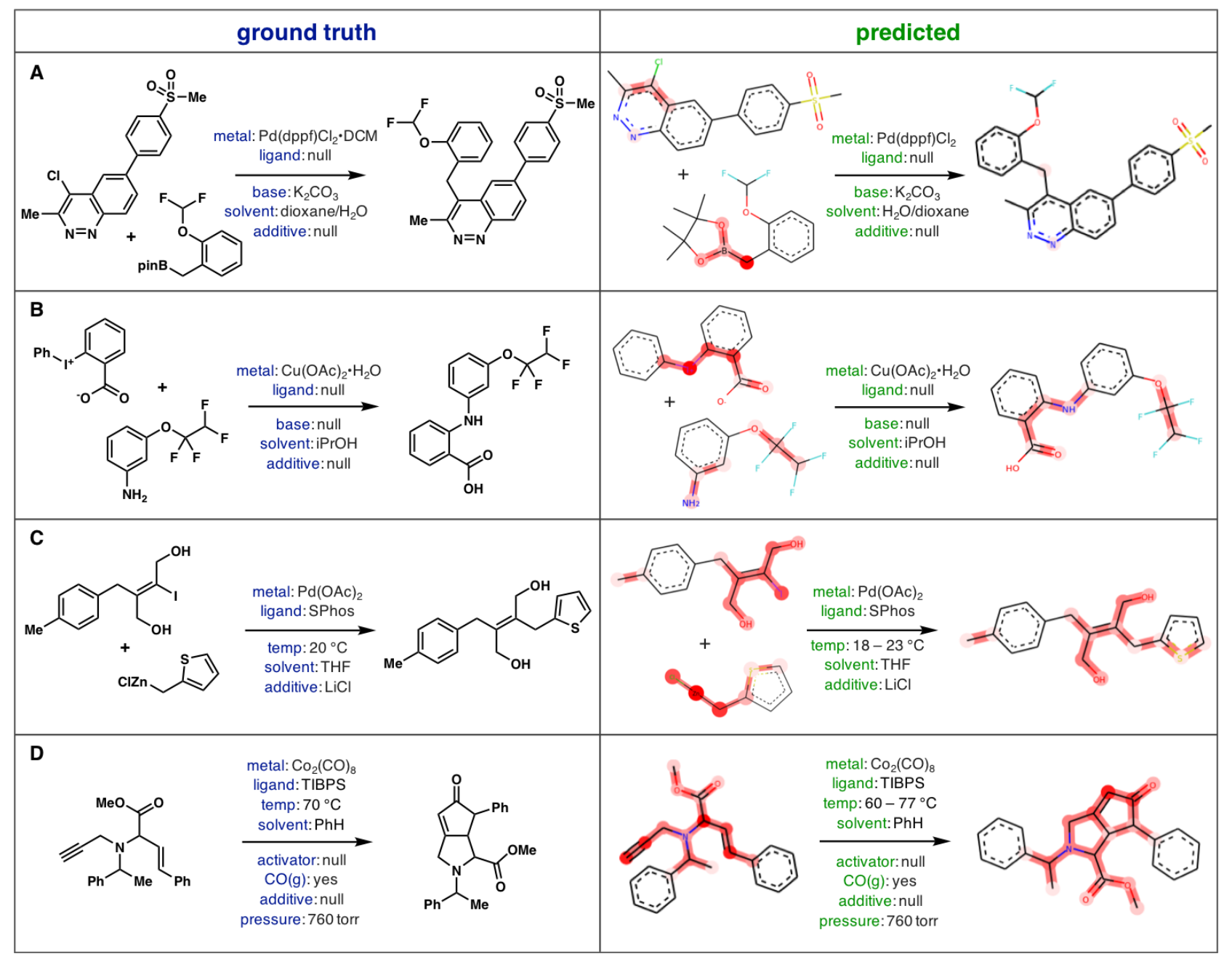

Figure 6: AR-GCN attention weight visualization and prediction examples from randomly chosen reactions in each dataset. Darker highlighting indicates higher attention.

model's assignment of importance on an atom, as they re-scale node features in the final graph layer before inference. When extracted, the weights can be mapped back onto a molecule's atoms and displayed by color scale using RDKit (Figure 1D). 57 This gives a visual interpretation of the functional groups most heavily informing the predictions. Example visualizations from a random reaction in each dataset and their AR-GCN predictions are included in Figure 6 , and several additional random examples for each reaction type can be found in the SI.

In the Suzuki example (Figure 6A), the attention is dominated by the $\mathrm{sp}^{3}$ carbon bearing the Bpin group, with additional contributions from the bis- $O$-substituted heteroaryl-chloride and its cinnoline nitrogen, all of which could be reasonably expected to influence reactivity. It is interesting that weights on the $o$-difluoromethoxy group, the sulfone, and the majority of the product are suppressed, perhaps indicating that an alkyl nucleophile is sufficient to predict the required conditions. The AR-GCN predictions are correct in each category besides the metal, where the model erroneously identifies the metal source $\mathrm{Pd}(\mathrm{dppf}) \mathrm{Cl}_{2}$ instead of its ground truth DCM adduct $\mathrm{Pd}(\mathrm{dppf}) \mathrm{Cl}_{2} \cdot \mathrm{DCM}$.

Conversely, the weights in the $\mathrm{C}-\mathrm{N}$ coupling example are more evenly distributed (Figure6B). Intuitively, the chemically active iodonium benzoate is given strong attention in the electrophile, as is the nucleophilic aniline nitrogen. Here, the $m$-tetrafluoroethoxy group is also weighted significantly and these groups are given similar attention in the product. All categories are predicted correctly in this example, though three of them are null.

The Negishi example (Figure 6 C) is an inter- 
esting $\mathrm{C}\left(\mathrm{sp}^{3}\right)-\mathrm{C}\left(\mathrm{sp}^{2}\right)$ coupling of a fully substituted alkenyl-iodide and thiophenyl-methylzinc chloride. Like with A, the strongest weights correspond to the $\mathrm{sp}^{3}$ nucleophilic carbon, though similarly strong attention is distributed over the electrophilic alkene including the pendant alcohols. These weights are again reflected in the product and all five condition categories are predicted correctly, including temperature and use of a $\mathrm{LiCl}$ additive.

Lastly, an intramolecular PKR (Figure 6D) showed the most uniformly distributed attention of the four examples. Still, the strongest weights are given to the participating alkyne and alkene, with additional emphasis on the amino ester bridging group. Weights are similarly distributed in the product, though strongest attention is intuitively assigned to the newly formed enone. Here, all 8 categories are predicted correctly including the use of an ambient carbon monoxide atmosphere $(C O(g)$ and pressure).

\subsection{Yield Analysis}

Having explored our models' chemical feature learning, we lastly investigated the effect of reaction yield, as it is a critical feature of synthesis data. Unsurprisingly, plotting the distribution of reaction yields in each dataset showed a uniformly strong bias towards high-yielding reactions (Figure 7A). Given the skewness of the data in this regard, we hypothesized that models would perform best at predicting conditions for high-yielding reactions.

We divided the dataset into quartiles by reaction yield and re-trained the AR-GCN with each sub-set, subsequently testing in each region and on the full test set (Figure $7 \mathrm{~B}$ ). Intuitively, models trained in any yield range tended to give highest accuracy when tested in the same range, occupying the confusion matrix diagonal in Figure 7B (top). To our surprise, however, the standard model trained on the full dataset gave consistently high accuracies, regardless of the test set (bottom row).

Since the yield bins contain varying amounts of data, we re-split the dataset, again ordered by yield but with equal sub-set sizes (Figure $7 \mathrm{~B}$ bottom). A similar trend was observed where the

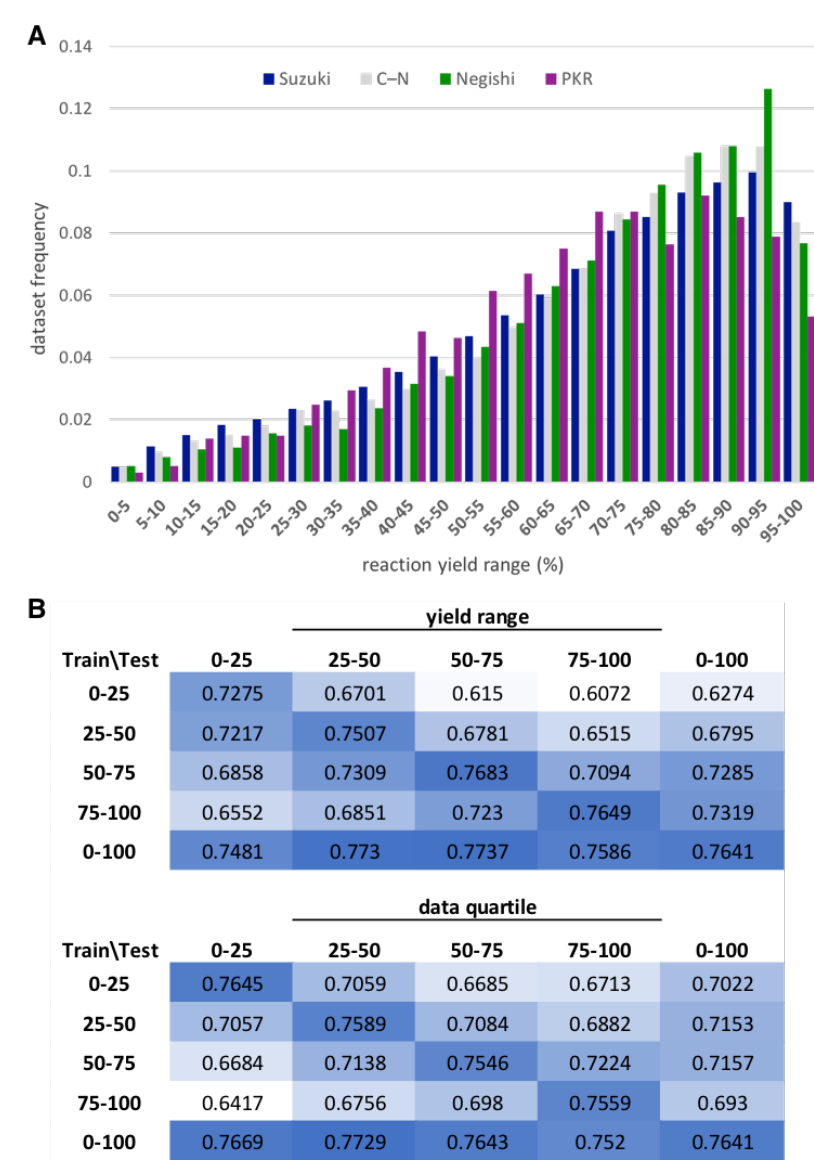

Figure 7: Performance dependence on reaction yield. A) Distribution of reaction yields for the four datasets. B) AR-GCN average top- $1 A_{c}$ values for Suzuki predictions when trained and tested in different yield ranges (top) and dataset quartiles arranged by yield (bottom).

highest accuracies were found on the diagonal and bottom row of the confusion matrix. Interestingly, the worst performing model was that trained in the highest yield range and tested in the lowest. We recognize that making "inaccurate" predictions on low-yielding reactions offers an avenue for predictive reaction optimization and future studies will explore this objective.

\section{Conclusion and Outlook}

In summary, we present a multi-label classification approach to predicting experimental reaction conditions for organic synthesis. We successfully model four high-value reaction types using expert-crafted label dictionaries: Suzuki, C-N, and Negishi couplings, and Pauson-Khand 
reactions. We explore and optimize two model classes: gradient boosting machines and graph convolutional networks. We find that GCN models perform very well in larger datasets, while GBMs show success for smaller datasets.

We report the first use of classifier trellises in molecular machine learning, and find that they are able to incorporate label correlations in modeling. We introduce a novel reactionlevel graph attention mechanism that provides significant accuracy gains when coupled with relational GCNs, and construct a hybrid GCN architecture called attended relational GCNs, or AR-GCNs. We further provide an analytical framework for the chemical interpretation of our models, extracting the trellis structures and mutual information matrices of the CT-GBMs, and visualizing the attention weights assigned in AR-GCN predictions.

Experimental studies are currently underway assessing the feasibility of model predictions on novel reactions. Additionally, efforts to apply our modeling framework to less-structured reaction types such as oxidations and reductions are ongoing. Future studies will address the interplay between structure representation and classifier chaining, as well as the extension of our reaction attention mechanism to other tasks. We expect the work herein to be very informative for future condition prediction studies, a highly valuable but underexplored learning task.

Acknowledgement We thank Prof Pietro Perona for mentorship guidance and helpful project discussions, and Chase Blagden for help structuring the GBM experiments. Fellowship support was provided by the NSF (M.R.M., T.J.D Grant No. DGE- 1144469). S.E.R. is a Heritage Medical Research Institute Investigator. Financial support from Research Corporation is warmly acknowledged.

\section{Supporting Information Avail- able}

This will usually read something like: "Experimental procedures and characterization data for all new compounds. The class will automati- cally add a sentence pointing to the information on-line:

\section{References}

(1) Dreher, S. D. Catalysis in medicinal chemistry. Reaction Chemistry \& Engineering 2019, 4, 1530-1535.

(2) Blakemore, D. C.; Castro, L.; Churcher, I.; Rees, D. C.; Thomas, A. W.; Wilson, D. M.; Wood, A. Organic synthesis provides opportunities to transform drug discovery. Nature Chemistry 2018, 10, 383394.

(3) Mahatthananchai, J.; Dumas, A. M.; Bode, J. W. Catalytic Selective Synthesis. Angewandte Chemie International Edition 2012, 51, 10954-10990.

(4) Reid, J. P.; Sigman, M. S. Comparing quantitative prediction methods for the discovery of small-molecule chiral catalysts. $\mathrm{Na}$ ture Reviews Chemistry 2018, 2, 290-305.

(5) Butler, K. T.; Davies, D. W.; Cartwright, H.; Isayev, O.; Walsh, A. Machine learning for molecular and materials science. Nature 2018, 559, 547-555.

(6) Wu, Z.; Ramsundar, B.; Feinberg, E. N.; Gomes, J.; Geniesse, C.; Pappu, A. S.; Leswing, K.; Pande, V. MoleculeNet: a benchmark for molecular machine learning. Chemical Science 2018, 9, 513-530.

(7) Yang, K.; Swanson, K.; Jin, W.; Coley, C.; Eiden, P.; Gao, H.; Guzman-Perez, A.; Hopper, T.; Kelley, B.; Mathea, M.; Palmer, A.; Settels, V.; Jaakkola, T.; Jensen, K.; Barzilay, R. Analyzing Learned Molecular Representations for Property Prediction. Journal of Chemical Information and Modeling 2019, 59, 3370-3388.

(8) Withnall, M.; Lindelöf, E.; Engkvist, O.; Chen, H. Building attention and edge message passing neural networks for bioactivity 
and physical-chemical property prediction. Journal of Cheminformatics 2020, 12.

(9) Stokes, J. M. et al. A Deep Learning Approach to Antibiotic Discovery. Cell 2020, 180, 688-702.e13.

(10) Blaschke, T.; Olivecrona, M.; Engkvist, O.; Bajorath, J.; Chen, H. Application of Generative Autoencoder in De Novo Molecular Design. Molecular Informatics 2018, 37, 1700123.

(11) Elton, D. C.; Boukouvalas, Z.; Fuge, M. D.; Chung, P. W. Deep learning for molecular design - a review of the state of the art. Molecular Systems Design \& Engineering 2019, 4, 828-849.

(12) Prykhodko, O.; Johansson, S. V.; Kotsias, P.-C.; Arús-Pous, J.; Bjerrum, E. J.; Engkvist, O.; Chen, H. A de novo molecular generation method using latent vector based generative adversarial network. Journal of Cheminformatics 2019, 11, 74.

(13) Moret, M.; Friedrich, L.; Grisoni, F.; Merk, D.; Schneider, G. Generating Customized Compound Libraries for Drug Discovery with Machine Intelligence. 2019,

(14) Panteleev, J.; Gao, H.; Jia, L. Recent applications of machine learning in medicinal chemistry. Bioorganic \&6 Medicinal Chemistry Letters 2018, 28, 2807-2815.

(15) Skoraczyński, G.; Dittwald, P.; Miasojedow, B.; Szymkuć, S.; Gajewska, E. P.; Grzybowski, B. A.; Gambin, A. Predicting the outcomes of organic reactions via machine learning: are current descriptors sufficient? Scientific Reports 2017, 7.

(16) Coley, C. W.; Barzilay, R.; Jaakkola, T. S.; Green, W. H.; Jensen, K. F. Prediction of Organic Reaction Outcomes Using Machine Learning. ACS Central Science 2017, 3, 434-443.

(17) Ahneman, D. T.; Estrada, J. G.; Lin, S.; Dreher, S. D.; Doyle, A. G. Predicting reaction performance in $\mathrm{C}-\mathrm{N}$ cross-coupling using machine learning. Science 2018, 360, 186-190.

(18) Nielsen, M. K.; Ahneman, D. T.; Riera, O.; Doyle, A. G. Deoxyfluorination with Sulfonyl Fluorides: Navigating Reaction Space with Machine Learning. Journal of the American Chemical Society 2018, 140, 5004-5008.

(19) Simón-Vidal, L.; García-Calvo, O.; Oteo, U.; Arrasate, S.; Lete, E.; Sotomayor, N.; González-Díaz, H. Perturbation-Theory and Machine Learning (PTML) Model for High-Throughput Screening of Parham Reactions: Experimental and Theoretical Studies. Journal of Chemical Information and Modeling 2018, 58, 1384-1396.

(20) Granda, J. M.; Donina, L.; Dragone, V.; Long, D.-L.; Cronin, L. Controlling an organic synthesis robot with machine learning to search for new reactivity. Nature 2018, 559, 377-381.

(21) Hughes, T. B.; Miller, G. P.; Swamidass, S. J. Modeling Epoxidation of Druglike Molecules with a Deep Machine Learning Network. ACS Central Science 2015, 1, 168-180.

(22) Peng, Q.; Duarte, F.; Paton, R. S. Computing organic stereoselectivity - from concepts to quantitative calculations and predictions. Chemical Society Reviews 2016, 45, 6093-6107.

(23) Banerjee, S.; Sreenithya, A.; Sunoj, R. B. Machine learning for predicting product distributions in catalytic regioselective reactions. Physical Chemistry Chemical Physics 2018, 20, 18311-18318.

(24) Beker, W.; Gajewska, E. P.; Badowski, T.; Grzybowski, B. A. Prediction of Major Regio-, Site-, and Diastereoisomers in Diels-Alder Reactions by Using MachineLearning: The Importance of Physically Meaningful Descriptors. Angewandte Chemie International Edition 2019, 58, 4515-4519. 
(25) Zahrt, A. F.; Henle, J. J.; Rose, B. T.; Wang, Y.; Darrow, W. T.; Denmark, S. E. Prediction of higher-selectivity catalysts by computer-driven workflow and machine learning. Science 2019, 363, eaau5631.

(26) Coley, C. W.; Green, W. H.; Jensen, K. F. Machine Learning in Computer-Aided Synthesis Planning. Accounts of Chemical Research 2018, 51, 1281-1289.

(27) Segler, M. H. S.; Preuss, M.; Waller, M. P. Planning chemical syntheses with deep neural networks and symbolic AI. Nature 2018, 555, 604-610.

(28) Coley, C. W.; Green, W. H.; Jensen, K. F. RDChiral: An RDKit Wrapper for Handling Stereochemistry in Retrosynthetic Template Extraction and Application. Journal of Chemical Information and Modeling 2019, 59, 2529-2537.

(29) Badowski, T.; Gajewska, E. P.; Molga, K.; Grzybowski, B. A. Synergy Between Expert and Machine-Learning Approaches Allows for Improved Retrosynthetic Planning. Angewandte Chemie International Edition 2020, 59, 725-730.

(30) Nicolaou, C. A.; Watson, I. A.; LeMasters, M.; Masquelin, T.; Wang, J. Context Aware Data-Driven Retrosynthetic Analysis. Journal of Chemical Information and Modeling 2020,

(31) Gao, H.; Struble, T. J.; Coley, C. W.; Wang, Y.; Green, W. H.; Jensen, K. F. Using Machine Learning To Predict Suitable Conditions for Organic Reactions. ACS Central Science 2018, 4, 1465-1476.

(32) Ryou*, S.; Maser*, M. R.; Cui*, A. Y.; DeLano, T. J.; Yue, Y.; Reisman, S. E. Graph Neural Networks for the Prediction of Substrate-Specific Organic Reaction Conditions. arXiv:2007.04275 [cs, LG] 2020,

(33) Huerta, F.; Hallinder, S.; Minidis, A. Machine Learning to Reduce Reaction Optimization Lead Time - Proof of Concept with Suzuki, Negishi and Buchwald-Hartwig Cross-Coupling Reactions; preprint ChemRxiv.12613214, 2020 .

(34) Schlichtkrull, M.; Kipf, T. N.; Bloem, P.; Berg, R. v. d.; Titov, I.; Welling, M. Modeling Relational Data with Graph Convolutional Networks. arXiv:1703.06103 [cs, stat] 2017,

(35) Friedman, J. H. Greedy Function Approximation: A Gradient Boosting Machine. The Annals of Statistics 2001, 29, 11891232 .

(36) Reaxys. https://new.reaxys.com/, (accessed on May 13, 2019).

(37) Weininger, D. SMILES, a chemical language and information system. 1. Introduction to methodology and encoding rules. Journal of Chemical Information and Modeling 1988, 28, 31-36.

(38) Cui, Y.; Jia, M.; Lin, T.-Y.; Song, Y.; Belongie, S. Class-Balanced Loss Based on Effective Number of Samples. arXiv:1901.05555 [cs] 2019,

(39) Wu, X.-Z.; Zhou, Z.-H. A Unified View of Multi-Label Performance Measures. arXiv:1609.00288 [cs] 2017,

(40) Natekin, A.; Knoll, A. Gradient boosting machines, a tutorial. Frontiers in Neurorobotics 2013, 7 .

(41) Ke, G.; Meng, Q.; Finley, T.; Wang, T.; Chen, W.; Ma, W.; Ye, Q.; Liu, T.-Y. In Advances in Neural Information Processing Systems 30; Guyon, I., Luxburg, U. V., Bengio, S., Wallach, H., Fergus, R., Vishwanathan, S., Garnett, R., Eds.; Curran Associates, Inc., 2017; pp 3146-3154.

(42) Moriwaki, H.; Tian, Y.-S.; Kawashita, N.; Takagi, T. Mordred: a molecular descriptor calculator. Journal of Cheminformatics 2018, 10, 4.

(43) Breiman, L. Random Forests. Machine Learning 2001, 45, 5-32. 
(44) Zhang, M.-L.; Zhou, Z.-H. A Review on Multi-Label Learning Algorithms. IEEE Transactions on Knowledge and Data Engineering 2014, 26, 1819-1837.

(45) Read, J.; Pfahringer, B.; Holmes, G.; Frank, E. Classifier Chains for Multilabel Classification. Machine Learning and Knowledge Discovery in Databases. Berlin, Heidelberg, 2009; pp 254-269.

(46) Read, J.; Martino, L.; Olmos, P.; Luengo, D. Scalable Multi-Output Label Prediction: From Classifier Chains to Classifier Trellises. Pattern Recognition 2015, 48, 2096-2109.

(47) Gilmer, J.; Schoenholz, S. S.; Riley, P. F.; Vinyals, O.; Dahl, G. E. Neural Message Passing for Quantum Chemistry. arXiv:1704.01212 [cs] 2017,

(48) Veličković, P.; Cucurull, G.; Casanova, A.; Romero, A.; Liò, P.; Bengio, Y. Graph Attention Networks. arXiv:1710.10903 [cs, stat] 2018,

(49) Busbridge, D.; Sherburn, D.; Cavallo, P.; Hammerla, N. Y. Relational Graph Attention Networks. arXiv:1904.05811 [cs, stat] 2019 ,

(50) Tokui, S.; Oono, K.; Hido, S.; Clayton, J. Chainer: a Next-Generation Open Source Framework for Deep Learning. 2015,

(51) Newman, M. E. J. Power laws, Pareto distributions and Zipf's law. Contemporary Physics 2005, 46, 323-351.

(52) Zhou, K.; Dong, Y.; Lee, W. S.; Hooi, B.; $\mathrm{Xu}, \mathrm{H}$;; Feng, J. Effective Training Strategies for Deep Graph Neural Networks. arXiv:2006.07107 [cs, stat] 2020,

(53) Galvez, J.; Garcia, R.; Salabert, M. T.; Soler, R. Charge Indexes. New Topological Descriptors. Journal of Chemical Information and Modeling 1994, 34, 520-525.
(54) Moreau, G.; Broto, P. The Autocorrelation of a Topological Structure: A New Molecular Descriptor. New Journal of Chemistry 1980, 4, 359-360.

(55) Hollas, B. An Analysis of the Autocorrelation Descriptor for Molecules. Journal of Mathematical Chemistry 2003, 33, 91-101.

(56) Chuang, K. V.; Keiser, M. J. Adversarial Controls for Scientific Machine Learning. ACS Chemical Biology 2018, 13, 28192821.

(57) Landrum, G. A. RDKit: Open-Source Cheminformatics Software. (accessed Nov 20, 2016). 


\section{Graphical TOC Entry}

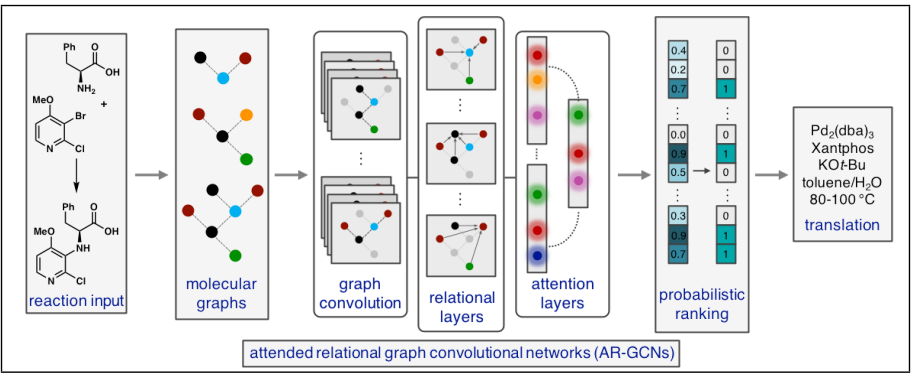




\section{Supporting Information:}

\section{Multi-Label Classification Models for the \\ Prediction of Cross-Coupling Reaction Conditions}

Michael R. Maser, ${ }^{\dagger, \S}$ Alexander Y. Cui, ${ }^{\ddagger}, \S$ Serim Ryou, ${ }^{\mathbf{9}, \S}$ Travis J. DeLano, ${ }^{\dagger}$ Yisong Yue,$^{\ddagger}$ and Sarah E. Reisman*,†

$\dagger$ Division of Chemistry and Chemical Engineering, California Institute of Technology,

Pasadena, California, USA

$\ddagger$ Department of Computing and Mathematical Sciences, California Institute of Technology,

Pasadena, California, USA

\Computational Vision Lab, California Institute of Technology, Pasadena, California, USA

$\S$ Equal contribution.

E-mail: reisman@caltech.edu

\section{S1 Data preparation and reaction dictionaries}

Full procedures for data processing are outlined in our previous preprint. $\underline{\text { S1 }}$ An example protocol with full code is included in the associated github repository: https://github.com /slryou41/reaction-gcnn.git in the path: data/data_processing_example.ipynb. The worked example includes procedures for sorting reagents into categories by reaction role and aggregating into a full reaction dictionary. Final dictionaries for all four datasets as .csv files can be found in the repository path: data/all_dictionaries/, and are tabulated below. 
Table S1: Suzuki dataset dictionary.

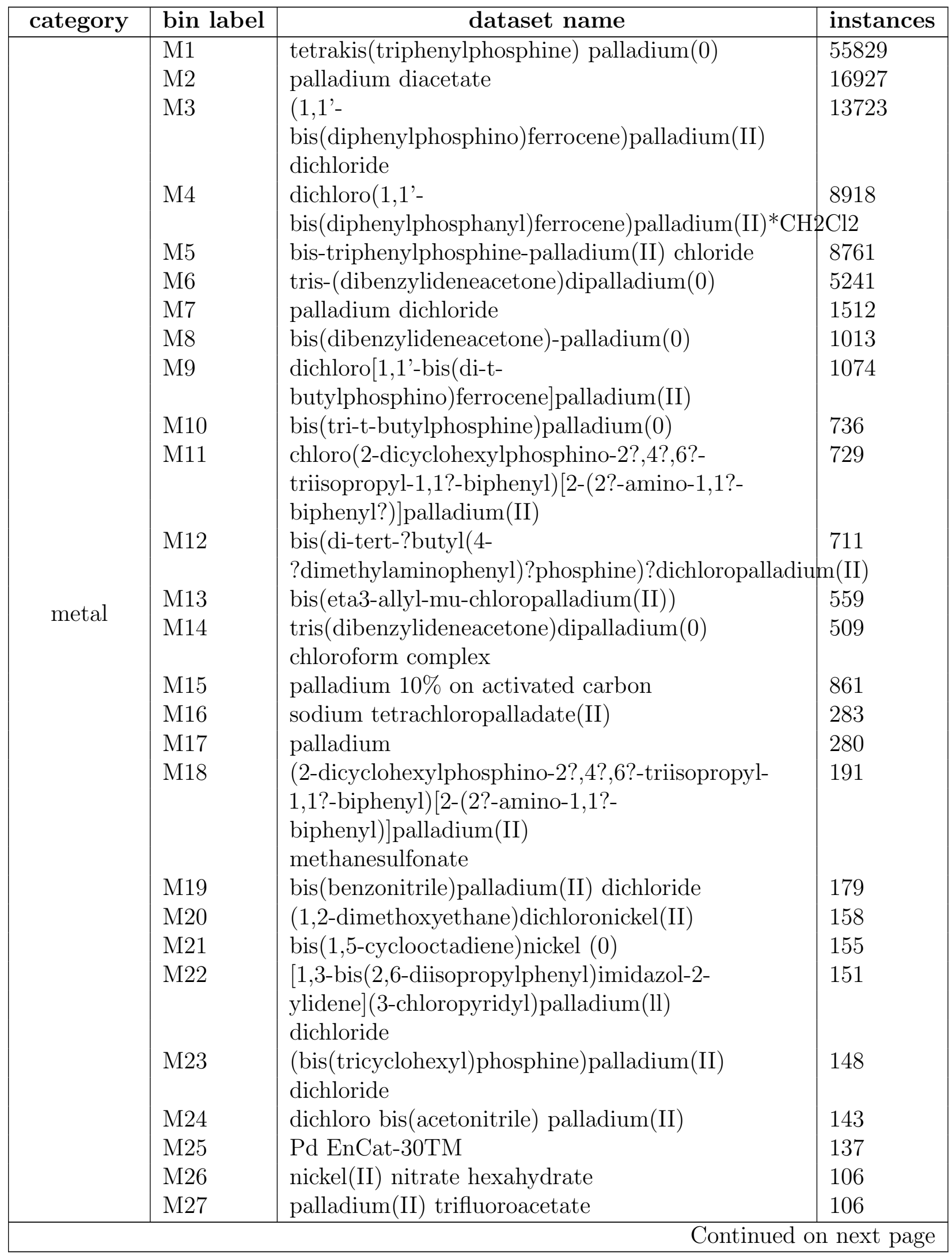


Table S1 - continued from previous page

\begin{tabular}{|c|c|c|c|}
\hline \multirow[t]{2}{*}{ category } & bin label & dataset name & instances \\
\hline & M28 & $\begin{array}{l}\text { dichlorobis[1- } \\
\text { (dicyclohexylphosphanyl)piperidine]palladium(II) }\end{array}$ & 102 \\
\hline \multirow{37}{*}{ ligand } & L1 & triphenylphosphine & 4489 \\
\hline & L2 & $\begin{array}{l}\text { dicyclohexyl-(2',6'-dimethoxybiphenyl-2-yl)- } \\
\text { phosphane }\end{array}$ & 3163 \\
\hline & L3 & XPhos & 2100 \\
\hline & L4 & tricyclohexylphosphine & 1808 \\
\hline & L5 & tris-(o-tolyl)phosphine & 902 \\
\hline & L6 & tri-tert-butyl phosphine & 694 \\
\hline & L7 & tri tert-butylphosphoniumtetrafluoroborate & 616 \\
\hline & L8 & trisodium tris(3-sulfophenyl)phosphine & 556 \\
\hline & L9 & 1,1'-bis-(diphenylphosphino)ferrocene & 486 \\
\hline & L10 & 4,5-bis(diphenylphos4,5-bis(diphenylphosphino)- & 424 \\
\hline & & 9,9-dimethylxanthenephino)-9,9-dimethylxanthene & \\
\hline & L11 & CyJohnPhos & 370 \\
\hline & L12 & ruphos & 293 \\
\hline & L13 & 1,3,5,7-tetramethyl-8-phenyl-2,4,6-trioxa-8- & 279 \\
\hline & L14 & tricyclohexylphosphine tetrafluoroborate & 240 \\
\hline & L15 & johnphos & 223 \\
\hline & L16 & 4,4'-di-tert-butyl-2,2'-bipyridine & 216 \\
\hline & L17 & catacxium A & 192 \\
\hline & L18 & trifuran-2-yl-phosphane & 183 \\
\hline & L19 & triphenyl-arsane & 182 \\
\hline & L20 & 1,1'-bis(di-tertbutylphosphino)ferrocene & 142 \\
\hline & L21 & 2,2'-bis-(diphenylphosphino)-1,1'-binaphthyl & 129 \\
\hline & L22 & Tedicyp & 218 \\
\hline & L23 & bis[2-(diphenylphosphino)phenyl] ether & 108 \\
\hline & B1 & potassium carbonate & 48981 \\
\hline & B2 & sodium carbonate & 39769 \\
\hline & B3 & potassium phosphate & 17799 \\
\hline & B4 & caesium carbonate & 13345 \\
\hline & B5 & sodium hydrogencarbonate & 3722 \\
\hline & B6 & cesium fluoride & 2810 \\
\hline & B7 & sodium hydroxide & 2156 \\
\hline & B8 & potassium hydroxide & 2155 \\
\hline & B9 & potassium fluoride & 2097 \\
\hline & B10 & triethylamine & 1370 \\
\hline & B11 & potassium phosphate tribasic trihydrate & 1016 \\
\hline & B12 & potassium acetate & 931 \\
\hline & \multicolumn{3}{|c|}{ potassium tert-butylate } \\
\hline
\end{tabular}


Table S1 - continued from previous page

\begin{tabular}{|c|c|c|c|}
\hline category & bin label & dataset name & instances \\
\hline \multirow{22}{*}{ base } & B14 & potassium phosphate monohydrate & 826 \\
\hline & B15 & sodium acetate & 418 \\
\hline & B16 & sodium t-butanolate & 392 \\
\hline & B17 & barium dihydroxide & 374 \\
\hline & B18 & N-ethyl-N,N-diisopropylamine & 336 \\
\hline & B19 & lithium hydroxide & 321 \\
\hline & B20 & potassium phosphate tribasic heptahydrate & 317 \\
\hline & B21 & diisopropylamine & 209 \\
\hline & B22 & sodium methylate & 175 \\
\hline & B23 & tetrabutyl ammonium fluoride & 173 \\
\hline & B24 & barium hydroxide octahydrate & 171 \\
\hline & $\mathrm{B} 25$ & potassium dihydrogenphosphate & 166 \\
\hline & B26 & potassium fluoride dihydrate & 156 \\
\hline & B27 & 1,4-diaza-bicyclo[2.2.2] octane & 154 \\
\hline & B28 & lithium hydroxide monohydrate & 143 \\
\hline & B29 & tetra-butylammonium acetate & 137 \\
\hline & B30 & sodium phosphate & 133 \\
\hline & B31 & potassium hydrogencarbonate & 131 \\
\hline & B32 & dipotassium hydrogenphosphate & 127 \\
\hline & B33 & tripotassium phosphate $n$ hydrate & 123 \\
\hline & B34 & cesiumhydroxide monohydrate & 112 \\
\hline & B35 & sodium phosphate dodecahydrate & 103 \\
\hline \multirow{10}{*}{ solvent } & S1 & tetrahydrofuran & 18113 \\
\hline & $\mathrm{S} 2$ & ethanol & 24836 \\
\hline & S3 & methanol & 4374 \\
\hline & S4 & 1,4-dioxane & 39107 \\
\hline & S5 & 1,2-dimethoxyethane & 19131 \\
\hline & S6 & acetonitrile & 4366 \\
\hline & S7 & toluene & 28304 \\
\hline & S8 & $\mathrm{N}, \mathrm{N}$-dimethyl formamide & 15110 \\
\hline & S9 & water & 92175 \\
\hline & S10 & 1-methyl-pyrrolidin-2-one & 472 \\
\hline & A1 & tetrabutylammomium bromide & 3003 \\
\hline & A2 & water & 1606 \\
\hline & A3 & lithium chloride & 819 \\
\hline & A4 & hydrogenchloride & 780 \\
\hline & A5 & copper(l) iodide & 546 \\
\hline & A6 & silver(l) oxide & 405 \\
\hline & A7 & copper diacetate & 183 \\
\hline & A8 & dmap & 181 \\
\hline additive & A9 & Aliquat 336 & 169 \\
\hline
\end{tabular}


Table S1 - continued from previous page

\begin{tabular}{|l|l|l|l|}
\hline category & bin label & \multicolumn{1}{|c|}{ dataset name } & instances \\
\hline & A10 & cetyltrimethylammonim bromide & 167 \\
& A11 & copper(l) chloride & 164 \\
& A12 & potassium bromide & 157 \\
& A13 & trifluoroacetic acid & 151 \\
& A14 & oxygen & 148 \\
& A15 & air & 112 \\
& A16 & 18-crown-6 ether & 127 \\
& A17 & sodium dodecyl-sulfate & 113 \\
\hline
\end{tabular}

Table S2: C-N dataset dictionary.

\begin{tabular}{|l|l|l|l|}
\hline category & bin label & \multicolumn{1}{|c|}{ dataset name } & instances \\
\hline & M1 & copper(l) iodide & 8180 \\
& M2 & tris-(dibenzylideneacetone)dipalladium(0) & 6995 \\
& M3 & palladium diacetate & 4668 \\
& M4 & copper & 1875 \\
M5 & bis(dibenzylideneacetone)-palladium(0) & 1292 \\
& M6 & copper(I) oxide & 932 \\
M7 & copper(II) oxide & 402 \\
M8 & copper(l) chloride & 386 \\
M9 & copper(I) bromide & 348 \\
M10 & bis(eta3-allyl-mu-chloropalladium(II)) & 433 \\
M11 & copper(II) acetate monohydrate & 352 \\
M12 & (1,1'- & 159 \\
& & bis(diphenylphosphino)ferrocene)palladium(II) & \\
& dichloride & 181 \\
M13 & bis(tri-t-butylphosphine)palladium(0) & 116 \\
M14 & iron(III) chloride & 91 \\
M15 & copper(II) bis(trifluoromethanesulfonate) & 88 \\
M16 & copper(ll) bromide & 82 \\
M17 & bis-triphenylphosphine-palladium(II) chloride & 154 \\
M18 & copper(II) sulfate & 78 \\
M19 & bis(acetylacetonate)nickel(II) & 71 \\
M20 & palladium 10\% on activated carbon & 68 \\
M21 & tetrakis(triphenylphosphine) palladium(0) & 67 \\
M22 & dichlorobis(tri-O-tolylphosphine)palladium & 66 \\
M23 & (1,2-dimethoxyethane)dichloronickel(II) & 63 \\
M24 & palladium dichloride & 58 \\
M25 & copper(I) thiophene-2-carboxylate & 56 \\
M26 & cobalt(II) oxalate dihydrate & \\
& & Continued on next page \\
\hline
\end{tabular}


Table S2 - continued from previous page

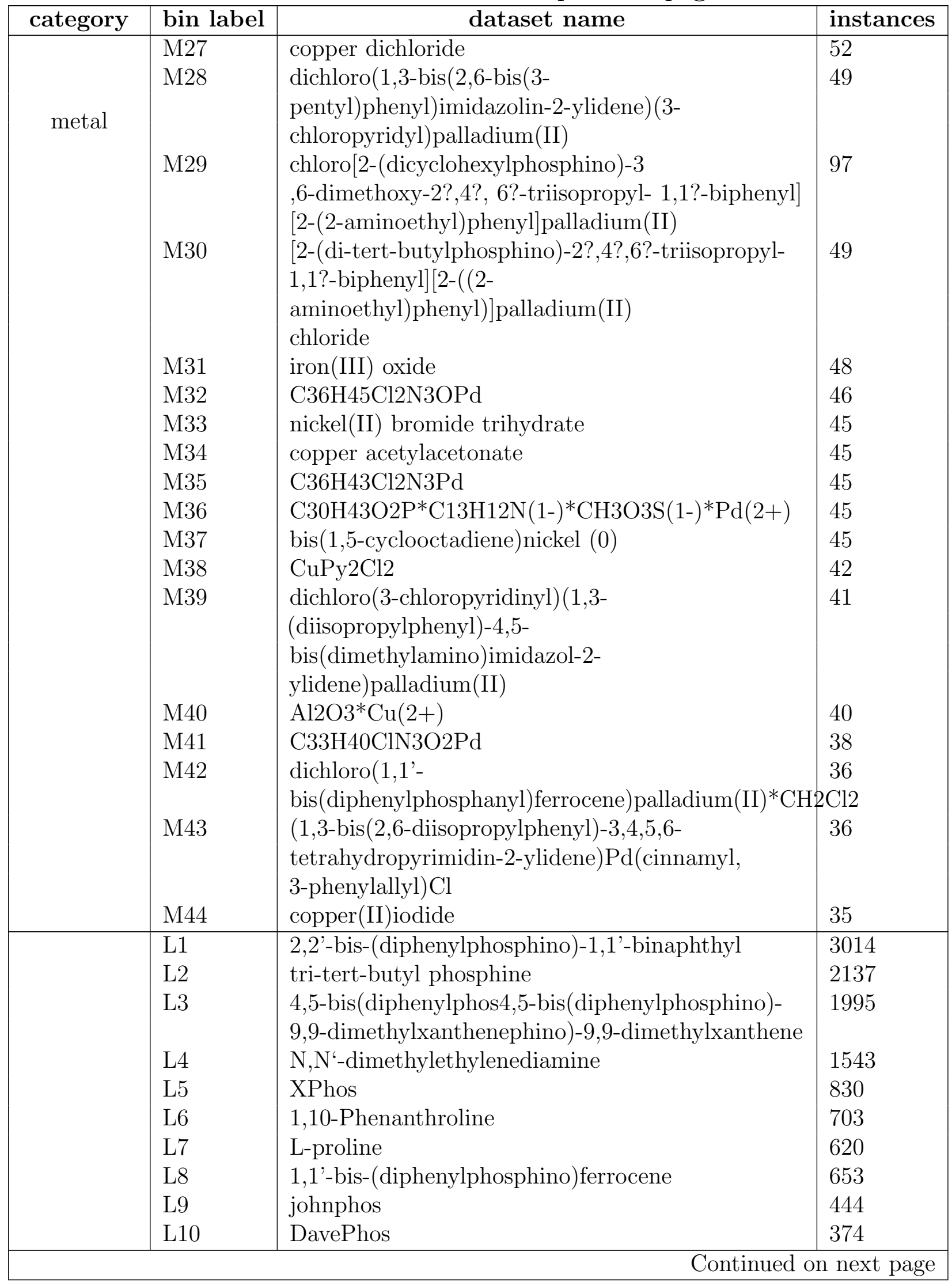


Table S2 - continued from previous page

\begin{tabular}{|c|c|c|c|}
\hline category & bin label & dataset name & instances \\
\hline \multirow{34}{*}{ ligand } & L11 & triphenylphosphine & 275 \\
\hline & L12 & ruphos & 266 \\
\hline & L13 & tri tert-butylphosphoniumtetrafluoroborate & 265 \\
\hline & L14 & tert-butyl XPhos & 242 \\
\hline & L15 & dicyclohexyl-(2',6'-dimethoxybiphenyl-2-yl)- & 261 \\
\hline & & phosphane & \\
\hline & L16 & trans-1,2-Diaminocyclohexane & 724 \\
\hline & L17 & 8-quinolinol & 206 \\
\hline & L18 & CyJohnPhos & 192 \\
\hline & L19 & trans-N,N'-dimethylcyclohexane-1,2-diamine & 535 \\
\hline & L20 & ethylenediamine & 175 \\
\hline & L21 & dimethylaminoacetic acid & 167 \\
\hline & L22 & dicyclohexyl[3,6-dimethoxy-2?,4?,6?-tris(1- & 165 \\
\hline & & methylethyl)[1,1?-biphenyl]-2-yl]phosphine & \\
\hline & L23 & 2,2,6,6-tetramethylheptane-3,5-dione & 163 \\
\hline & L24 & 1,1'-bi-2-naphthol & 162 \\
\hline & L25 & bis[2-(diphenylphosphino)phenyl] ether & 170 \\
\hline & L26 & $\begin{array}{l}\text { 1-dicyclohexylphosphino-2-di-tert- } \\
\text { butvlphosphinoethylferrocene }\end{array}$ & 142 \\
\hline & L27 & $\mathrm{P}(\mathrm{i}-\mathrm{BuNCH} 2) 3 \mathrm{CMe}$ & 110 \\
\hline & L28 & $\begin{array}{l}\text { di-tert-butyl2?-isopropoxy-[1,1?-binaphthalen]-2- } \\
\text { vlphosphane }\end{array}$ & 108 \\
\hline & L29 & di-tert-butyl (2,2-diphenyl-1-methyl-1- & 104 \\
\hline & L30 & $\begin{array}{l}\text { cyclopropyl)phosphine } \\
\mathrm{P}(\mathrm{i}-\mathrm{B} u \mathrm{NCH} 2 \mathrm{CH} 2) 3 \mathrm{~N}\end{array}$ & \\
\hline & L30 & $\mathrm{P}(\mathrm{i}-\mathrm{BuNCH} 2 \mathrm{CH} 2) 3 \mathrm{~N}$ & 98 \\
\hline & L31 & N,N-dimethylglycine hydrochoride & 96 \\
\hline & L32 & $\begin{array}{l}\text { N-[2-(di(1- } \\
\text { adamantyl)phosphino)phenvl]morpholine }\end{array}$ & 92 \\
\hline & L33 & $\begin{array}{l}\text { 5-(di-tert-butylphosphino)-1?, 3?, } \\
\text { 5?-triphenyl-1?H-[1,4?]bipyrazole }\end{array}$ & 91 \\
\hline & L34 & $\begin{array}{l}2 \text {-[2-(dicyclohexylphosphino)-phenyl]-1-methyl-1H- } \\
\text { indole }\end{array}$ & 86 \\
\hline & L35 & 4,4'-di-tert-butyl-2,2'-bipyridine & 85 \\
\hline & L36 & tris-(o-tolyl)phosphine & 77 \\
\hline & L37 & 2,8,9-tris(2-methylpropyl)-2,5,8,9-tetraaza-1- & 75 \\
\hline & L38 & cis-N,N'-dimethyl-1,2-diaminocyclohexane & 74 \\
\hline & L39 & monophosphine 1,2,3,4,5-pentaphenyl-1'-(di-tert- & 55 \\
\hline & L40 & $\begin{array}{l}\text { 5-(di(adamantan-1-yl)phosphino)-1?,3?,5?- } \\
\text { triphenvl-1?H-1 4?-bipvrazole }\end{array}$ & 55 \\
\hline & L41 & t-BuBrettPhos & 53 \\
\hline
\end{tabular}


Table S2 - continued from previous page

\begin{tabular}{|c|c|c|c|}
\hline \multirow[t]{10}{*}{ category } & bin label & dataset name & instances \\
\hline & L42 & 2-(N,N-dimethylamino)athanol & 53 \\
\hline & L43 & tricyclohexylphosphine & 46 \\
\hline & L44 & (E)-3-(dimethylamino)-1-(2-hydroxyphenyl)prop- & 46 \\
\hline & & 2-en-1-one & \\
\hline & L45 & di-tert-butylneopentylphosphonium & 38 \\
\hline & & tetrafluoroborate & \\
\hline & L46 & 2-di-tertbutylphosphino-3,4,5,6-tetramethyl- & 37 \\
\hline & & 2',4',6'-triisopropyl-1,1'-biphenyl & \\
\hline & L47 & $\mathrm{N}, \mathrm{N}, \mathrm{N}, \mathrm{N}$,-tetramethylethylenediamine & 26 \\
\hline \multirow{13}{*}{ base } & B1 & sodium t-butanolate & 9103 \\
\hline & B2 & potassium carbonate & 7129 \\
\hline & B3 & caesium carbonate & 6957 \\
\hline & B4 & potassium phosphate & 3274 \\
\hline & B5 & potassium tert-butylate & 2167 \\
\hline & B6 & potassium hydroxide & 1420 \\
\hline & B7 & triethylamine & 500 \\
\hline & B8 & lithium hexamethyldisilazane & 432 \\
\hline & B9 & sodium hydroxide & 430 \\
\hline & B10 & sodium hydride & 228 \\
\hline & B11 & sodium carbonate & 200 \\
\hline & B12 & potassium phosphate monohydrate & 130 \\
\hline & B13 & sodium hydrogencarbonate & 128 \\
\hline \multirow{19}{*}{ solvent } & S1 & toluene & 11970 \\
\hline & $\mathrm{S} 2$ & 1,4-dioxane & 5273 \\
\hline & S3 & N,N-dimethyl-formamide & 4246 \\
\hline & $\mathrm{S} 4$ & dimethyl sulfoxide & 3790 \\
\hline & $\mathrm{S} 5$ & water & 2464 \\
\hline & S6 & tetrahydrofuran & 1457 \\
\hline & S7 & 1,2-dimethoxyethane & 878 \\
\hline & S8 & tert-butyl alcohol & 841 \\
\hline & S9 & acetonitrile & 780 \\
\hline & S10 & ethanol & 549 \\
\hline & S11 & 5,5-dimethyl-1,3-cyclohexadiene & 497 \\
\hline & $\mathrm{S} 12$ & isopropyl alcohol & 316 \\
\hline & S13 & nitrobenzene & 315 \\
\hline & S14 & 1-methyl-pyrrolidin-2-one & 292 \\
\hline & S15 & hexane & 286 \\
\hline & S16 & N,N-dimethyl acetamide & 281 \\
\hline & S17 & 1,2-dichloro-benzene & 254 \\
\hline & S18 & neat (no solvent) & 240 \\
\hline & \multicolumn{3}{|c|}{ o-xylene } \\
\hline
\end{tabular}


Table S2 - continued from previous page

\begin{tabular}{|c|c|c|c|}
\hline \multirow[t]{4}{*}{ category } & bin label & dataset name & instances \\
\hline & S20 & xylene & 208 \\
\hline & S21 & methanol & 180 \\
\hline & $\mathrm{S} 22$ & ethyl acetate & 163 \\
\hline & A1 & 18-crown-6 ether & 455 \\
\hline & A2 & tetrabutylammomium bromide & 372 \\
\hline & A3 & 8-quinolinol & 206 \\
\hline & A4 & dimethylaminoacetic acid & 167 \\
\hline & A5 & 1,1'-bi-2-naphthol & 162 \\
\hline & A6 & water & 160 \\
\hline & A7 & sodium sulfate & 132 \\
\hline & A8 & 2-(2-methyl-1-oxopropyl)cyclohexanone & 121 \\
\hline & A9 & phenylboronic acid & 120 \\
\hline & $\mathrm{A} 10$ & 1,3-bis $[(2,6$-diisopropyl $)$ phenyl $]$ imidazolinium & 109 \\
\hline & & chloride & \\
\hline & A11 & potassium iodide & 108 \\
\hline & A12 & hydrogenchloride & 107 \\
\hline & A13 & ethylene glycol & 102 \\
\hline & A14 & N,N-dimethylglycine hydrochoride & 96 \\
\hline & A15 & 1,3-bis[2,6-diisopropylphenyl]imidazolium chloride & 95 \\
\hline & A16 & N-ethylmorpholine & 93 \\
\hline & A17 & tert-butyl alcohol & 87 \\
\hline & A18 & aluminum oxide & 84 \\
\hline & A19 & D-glucose & 83 \\
\hline & $\mathrm{A} 20$ & cetyltrimethylammonim bromide & 71 \\
\hline & $\mathrm{A} 21$ & 1,3-dimethyl-3,4,5,6-tetrahydro- $2(1 \mathrm{H})$ - & 68 \\
\hline & A 22 & pyrimidinone & 63 \\
\hline & A23 & $\begin{array}{l}N, N \text {-diphenyl-1H-pyrrole-2-carbonydrazıde } \\
\text { manganese(II) fluoride }\end{array}$ & 63 \\
\hline & $\mathrm{A} 24$ & dimethyl sulfoxide & 55 \\
\hline & A25 & 2-(N,N-dimethylamino)athanol & 53 \\
\hline & A26 & & 48 \\
\hline & A27 & iron(III) oxide & 48 \\
\hline & $\mathrm{A} 28$ & (E)-3-(dimethylamino)-1-(2-hydroxyphenyl)prop- & 46 \\
\hline & A29 & $\begin{array}{l}\text { 2-en-1-one } \\
\text { lithium bromide }\end{array}$ & 44 \\
\hline & A30 & 6,7-dihydro-5H-quinolin-8-one oxime & 43 \\
\hline & A31 & CVT-2537 & 42 \\
\hline & A32 & ammonium chloride & 42 \\
\hline & A33 & 1-methyl-pyrrolidin-2-one & 42 \\
\hline & A34 & tetra(n-butyl)ammonium hydroxide & 40 \\
\hline & A35 & salicylaldehyde-oxime & 39 \\
\hline & A36 & potassium fluoride on basic alumina & 39 \\
\hline
\end{tabular}


Table S2 - continued from previous page

\begin{tabular}{|c|c|c|c|}
\hline category & bin label & dataset name & instances \\
\hline \multirow{38}{*}{ additive } & A37 & toluene-4-sulfonic acid & 38 \\
\hline & A 38 & lithium chloride & 38 \\
\hline & A39 & pipecolic Acid & 37 \\
\hline & $\mathrm{A} 40$ & oxygen & 37 \\
\hline & A41 & metformin hydrochloride & 37 \\
\hline & $\mathrm{A} 42$ & 8-Hydroxyquinoline-N-oxide & 37 \\
\hline & $\mathrm{A} 43$ & 1-(5,6,7,8-tetrahydroquinolin-8-yl)ethan-1-one & 36 \\
\hline & A44 & tetrabutyl ammonium fluoride & 36 \\
\hline & A45 & N1,N2-bis(thiophen-2-ylmethyl)oxalamide & 36 \\
\hline & A46 & N-phenyl-2-pyridincarboxamide-1-oxide & 35 \\
\hline & A47 & $\mathrm{N}-((1$-oxy-pyridin-2-yl)methyl)oxalamic acid & 35 \\
\hline & A48 & C19H19N5O & 35 \\
\hline & A49 & manganese(II) chloride tetrahydrate & 34 \\
\hline & A50 & 1-tetralone oxime & 32 \\
\hline & A51 & N1,N2-bis(2,4,6-trimethoxyphenyl)oxalamide & 31 \\
\hline & A52 & N-methoxy-1H-pyrrole-2-carboxamide & 29 \\
\hline & A53 & ammonia & 29 \\
\hline & A54 & 1,2,3-Benzotriazole & 29 \\
\hline & A55 & dimethylenecyclourethane & 28 \\
\hline & A56 & isopropylmagnesium chloride & 27 \\
\hline & A57 & N-(2-cyanophenyl)pyridine-2-carboxamide & 27 \\
\hline & A58 & $\mathrm{C} 20 \mathrm{H} 18 \mathrm{~N} 2 \mathrm{O} 2$ & 27 \\
\hline & A59 & 2-acetylcyclohexanone & 27 \\
\hline & A60 & 2,6-di-tert-butyl-4-methyl-phenol & 26 \\
\hline & A61 & 2-hydroxy-pyridine N-oxide & 26 \\
\hline & A62 & TPGS-750-M & 25 \\
\hline & A63 & N?-phenyl-1H-pyrrole-2-carbohydrazide & 25 \\
\hline & A64 & lanthanum(III) oxide & 25 \\
\hline & A65 & ethylmagnesium bromide & 25 \\
\hline & A66 & ethyl 2-oxocyclohexane carboxylate & 25 \\
\hline & A67 & $\begin{array}{l}\text { 1,4-dimethyl-1,2,3,4-tetrahydro-5H- } \\
\text { benzo[e][1.4]diazepin-5-one }\end{array}$ & 25 \\
\hline & A68 & tetraethoxy orthosilicate & 24 \\
\hline & A69 & $\mathrm{N}, \mathrm{N}, \mathrm{N}^{\prime}, \mathrm{N}$ '-tetramethylguanidine & 24 \\
\hline & A70 & $\mathrm{C} 20 \mathrm{H} 26 \mathrm{~N} 4 \mathrm{O} 4$ & 24 \\
\hline & A71 & 2-methyl-8-quinolinol & 24 \\
\hline & A72 & 2-carbomethoxy-3-hydroxyquinoxaline-di-N-oxide & 24 \\
\hline & A73 & 1,3-diisopropyl-1H-imidazol-3-ium chloride & 24 \\
\hline & A74 & MOF-199 & 24 \\
\hline
\end{tabular}


Table S3: Negishi dataset dictionary.

\begin{tabular}{|c|c|c|c|}
\hline category & bin label & dataset name & instances \\
\hline \multirow{33}{*}{ metal } & M1 & tetrakis(triphenylphosphine) palladium(0) & 1902 \\
\hline & M2 & tris-(dibenzylideneacetone)dipalladium(0) & 572 \\
\hline & M3 & bis-triphenylphosphine-palladium(II) chloride & 418 \\
\hline & M4 & palladium diacetate & 370 \\
\hline & M5 & bis(dibenzylideneacetone)-palladium(0) & 344 \\
\hline & M6 & $\left(1,1^{\prime}-\right.$ & 334 \\
\hline & & $\begin{array}{l}\text { bis(diphenylphosphino)ferrocene)palladium(II) } \\
\text { dichloride }\end{array}$ & \\
\hline & M7 & bis(tri-t-butylphosphine)palladium(0) & 273 \\
\hline & M8 & dichloro(1,1'- & 248 \\
\hline & & \multicolumn{2}{|l|}{ bis(diphenylphosphanyl)ferrocene)palladium(II)*CH2Cl2 } \\
\hline & M9 & dichlorobis[1- & 168 \\
\hline & & (dicyclohexylphosphanyl)piperidine]palladium(II) & \\
\hline & M10 & palladium(l) tri-tert-butylphosphine iodide dimer & 101 \\
\hline & M11 & bis(tricyclohexylphosphine)nickel(II) dichloride & 99 \\
\hline & M12 & {$[(\mathrm{C} 10 \mathrm{H} 13-1,3-(\mathrm{CH} 2 \mathrm{P}(\mathrm{C} 6 \mathrm{H} 11) 2) 2) \mathrm{Pd}(\mathrm{Cl})]$} & 87 \\
\hline & M13 & $1,3-$ & 63 \\
\hline & & bis[(diphenylphosphino)propane]dichloronickel(II) & \\
\hline & M14 & bis $(1,5$-cyclooctadiene)nickel $(0)$ & 56 \\
\hline & M15 & nickel dichloride & 56 \\
\hline & M16 & tris(dibenzylideneacetone)dipalladium(0) & 46 \\
\hline & M17 & $\begin{array}{l}\text { chloroform complex } \\
\text { dichlorobis(tri-O-tolylphosphine)palladium }\end{array}$ & 46 \\
\hline & M18 & palladium & 44 \\
\hline & M19 & $\begin{array}{l}\text { [1,3-bis(2,6-diisopropylphenyl)imidazol-2- } \\
\text { ylidene](3chloro-pyridyl)palladium(II) } \\
\text { dichloride }\end{array}$ & 136 \\
\hline & M20 & $\mathrm{C} 20 \mathrm{H} 20 \mathrm{ClN} 3 \mathrm{Ni}$ & 42 \\
\hline & M21 & dichloro(1,3-bis $(2,6$-bis $(3-$ & 39 \\
\hline & & $\begin{array}{l}\text { pentyl)phenyl)imidazolin-2-ylidene)(3- } \\
\text { chloropyridyl)palladium(II) }\end{array}$ & \\
\hline & M22 & bis(triphenylphosphine)nickel(II) chloride & 38 \\
\hline & M23 & $\mathrm{C} 26 \mathrm{H} 24 \mathrm{ClN} 2 \mathrm{NiP}^{*} 0.1 \mathrm{C} 7 \mathrm{H} 8$ & 35 \\
\hline & M24 & cobalt(II) chloride & 34 \\
\hline & M25 & copper(I) bromide & 31 \\
\hline & M26 & $\mathrm{C} 40 \mathrm{H} 55 \mathrm{Cl} 5 \mathrm{~N} 3 \mathrm{Pd}$ & 30 \\
\hline & M27 & $\begin{array}{l}\text { [1,3-bis }(2,6 \text {-diisoheptylphenyl)-4,5- } \\
\text { dichloroimidazol-2-ylidene](3- } \\
\text { chloropyridyl)palladium(II) } \\
\text { dichloride }\end{array}$ & 29 \\
\hline & M28 & dichloro bis(acetonitrile) palladium(II) & 29 \\
\hline
\end{tabular}


Table S3 - continued from previous page

\begin{tabular}{|c|c|c|c|}
\hline category & bin label & dataset name & instances \\
\hline & M29 & palladium(II) trifluoroacetate & 27 \\
\hline & M30 & 1,2-bis(diphenylphosphino)ethane nickel(II) & 27 \\
\hline & & chloride & \\
\hline & M31 & $\mathrm{C} 27 \mathrm{H} 22 \mathrm{Cl} 2 \mathrm{~N} 3 \mathrm{NiP}$ & 24 \\
\hline & M32 & C38H34Br2N4Ni2P2 & 23 \\
\hline \multirow{24}{*}{ ligand } & L1 & 1,1'-bis-(diphenylphosphino)ferrocene & 233 \\
\hline & L2 & dicyclohexyl-(2',6'-dimethoxybiphenyl-2-yl)- & 196 \\
\hline & L3 & XPhos & 187 \\
\hline & L4 & triphenylphosphine & 161 \\
\hline & L5 & trifuran-2-yl-phosphane & 128 \\
\hline & L6 & monophosphine 1,2,3,4,5-pentaphenyl-1'-(di-tert- & 95 \\
\hline & & butylphosphino)ferrocene & \\
\hline & L7 & tris-(o-tolyl)phosphine & 70 \\
\hline & L8 & Ruphos & 61 \\
\hline & L9 & 2?-(dicyclohexylphophanyl)-N2,N2,N6,N6- & 37 \\
\hline & & tetramethyl[1,1?-biphenyl]-2,6-diamine & \\
\hline & L10 & tripiperidino-phosphine & 37 \\
\hline & L11 & tri tert-butylphosphoniumtetrafluoroborate & 35 \\
\hline & L12 & 1,2-bis-(dicyclohexylphosphino)ethane & 33 \\
\hline & L13 & 4,5-bis(diphenylphos4,5-bis(diphenylphosphino)- & 31 \\
\hline & & 9,9-dimethylxanthenephino)-9,9-dimethylxanthene & \\
\hline & L14 & N,N,N,N,-tetramethylethylenediamine & 24 \\
\hline & L15 & {$[2,2]$ bipyridinyl } & 22 \\
\hline & L16 & 4,4'-di-tert-butyl-2,2'-bipyridine & 21 \\
\hline & L17 & 1,2-Ph2-3,4-bis $(2,4,6-(\mathrm{t}-\mathrm{Bu}) 3-$ & 20 \\
\hline & & phenylphophinidene)cyclobutene & \\
\hline & L18 & johnphos & 20 \\
\hline & L19 & tri-tert-butyl phosphine & 19 \\
\hline & L20 & tricyclohexylphosphine & 18 \\
\hline \multirow{11}{*}{ temperature } & T1 & $-163-18$ & 101 \\
\hline & $\mathrm{T} 2$ & $18-23$ & 2313 \\
\hline & $\mathrm{T} 3$ & $23-50$ & 643 \\
\hline & $\mathrm{T} 4$ & $50-61$ & 975 \\
\hline & T5 & $61-80$ & 658 \\
\hline & T6 & $80-100$ & 673 \\
\hline & $\mathrm{T} 7$ & $100-120$ & 696 \\
\hline & $\mathrm{T} 8$ & $120-220$ & 479 \\
\hline & S1 & tetrahydrofuran & 4525 \\
\hline & $\mathrm{S} 2$ & N,N-dimethyl-formamide & 1003 \\
\hline & S3 & 1-methyl-pyrrolidin-2-one & 674 \\
\hline \multicolumn{4}{|c|}{ Continued on next page } \\
\hline
\end{tabular}


Table S3 - continued from previous page

\begin{tabular}{|c|c|c|c|}
\hline category & bin label & dataset name & instances \\
\hline \multirow{7}{*}{ solvent } & S4 & toluene & 541 \\
\hline & $\mathrm{S} 5$ & 1,4-dioxane & 335 \\
\hline & S6 & N,N-dimethyl acetamide & 247 \\
\hline & S7 & hexane & 219 \\
\hline & S8 & diethyl ether & 203 \\
\hline & S9 & water & 122 \\
\hline & $\mathrm{S} 10$ & 1,2-dimethoxyethane & 67 \\
\hline \multirow{31}{*}{ additive } & A1 & lithium chloride & 243 \\
\hline & A2 & zinc & 207 \\
\hline & A3 & copper(l) iodide & 154 \\
\hline & A4 & water & 62 \\
\hline & A5 & diisobutylaluminium hydride & 59 \\
\hline & A6 & tetrabutylammomium bromide & 52 \\
\hline & A7 & ammonium chloride & 51 \\
\hline & A8 & n-butyllithium & 46 \\
\hline & A9 & 1-Methylpyrrolidine & 42 \\
\hline & A10 & $\mathrm{Li} 2 \mathrm{CoCl} 4$ & 42 \\
\hline & A11 & sodium formate & 42 \\
\hline & A12 & hydrogenchloride & 36 \\
\hline & A13 & caesium carbonate & 36 \\
\hline & A14 & zinc diacetate & 32 \\
\hline & A15 & potassium carbonate & 30 \\
\hline & A16 & norborn-2-ene & 30 \\
\hline & A17 & lithium bromide & 28 \\
\hline & A18 & 1,3-dimethyl-3,4,5,6-tetrahydro-2 $(1 \mathrm{H})$ - & 23 \\
\hline & A19 & pyrmmanone & 22 \\
\hline & A 20 & 1-methyl-pyrrolidin-2-one & 21 \\
\hline & A21 & zinc(II) chloride & 21 \\
\hline & A22 & isoquinoline & 20 \\
\hline & A 23 & sodium carbonate & 19 \\
\hline & A 24 & 1-ethyl-2-pyrrolidinone & 18 \\
\hline & A25 & sodium & 16 \\
\hline & A26 & 1-methyl-1H-imidazole & 15 \\
\hline & A 27 & oxovanadium(V) ethoxydichloride & 12 \\
\hline & A28 & 2-(N,N-dimethylamino)athanol & 11 \\
\hline & A29 & [bdmim] [BF4] & 11 \\
\hline & A30 & $\begin{array}{l}\text { 1-butyl-2-(diphenylphosphanyl)-3- } \\
\text { methylimidazolium }\end{array}$ & 11 \\
\hline & & hexafluorophosphate & \\
\hline
\end{tabular}


Table S4: PKR dataset dictionary.

\begin{tabular}{|c|c|c|c|}
\hline category & bin label & dataset name & instances \\
\hline \multirow{18}{*}{ metal } & M1 & dicobalt octacarbonyl & 614 \\
\hline & M2 & di(rhodium)tetracarbonyl dichloride & 333 \\
\hline & M3 & chloro(1,5-cyclooctadiene)rhodium(I) dimer & 140 \\
\hline & M4 & {$[\mathrm{RhCl}(\mathrm{CO}) \mathrm{dppp}] 2$} & 92 \\
\hline & M5 & cobalt(II) bromide & 44 \\
\hline & M6 & palladium dichloride & 33 \\
\hline & M7 & dodecacarbonyl-triangulo-triruthenium & 32 \\
\hline & M8 & Co2Rh2 nanoparticles immobilized on charcoal & 50 \\
\hline & M9 & tetracobaltdodecacarbonyl & 44 \\
\hline & M10 & molybdenum hexacarbonyl & 23 \\
\hline & M11 & $\mathrm{Rh}(\mathrm{dppp}) 2 \mathrm{Cl}$ & 19 \\
\hline & M12 & cobalt nanoparticles on charcoal & 36 \\
\hline & M13 & methylidynetricobalt nonacarbonyl & 25 \\
\hline & M14 & bis(triphenylphosphine)(carbonyl)rhodium chloride & 11 \\
\hline & M15 & $\mathrm{PdCl}(\mathrm{OHNCCH} 3 \mathrm{C} 6 \mathrm{H} 4)(\mathrm{C} 5 \mathrm{H} 5 \mathrm{~N})$ & 10 \\
\hline & M16 & bis(1,5-cyclooctadiene)diiridium(I) dichloride & 9 \\
\hline & M17 & diiron nonacarbonyl & 9 \\
\hline & M18 & iron(II) bis(trimethylsilyl)amide & 9 \\
\hline \multirow{7}{*}{ ligand } & L1 & 1,1,3,3-tetramethyl-2-thiourea & 128 \\
\hline & L2 & 1,3-bis-(diphenylphosphino)propane & 93 \\
\hline & L3 & 2,2'-bis-(diphenylphosphino)-1,1'-binaphthyl & 31 \\
\hline & L4 & triphenylphosphine & 16 \\
\hline & L5 & tri-n-butylphosphine sulfide & 15 \\
\hline & L6 & (S)-3,5-di-tert-butyl-4-methoxyphenyl-(6,6?- & 12 \\
\hline & & $\begin{array}{l}\text { dimethoxybiphenyl-2,2?-diyl)- } \\
\text { bis(diphenylphosphine) }\end{array}$ & \\
\hline \multirow{14}{*}{ temperature } & T1 & $-98-20$ & 83 \\
\hline & $\mathrm{T} 2$ & 20 & 961 \\
\hline & T3 & $20-60$ & 299 \\
\hline & $\mathrm{T} 4$ & $60-77$ & 370 \\
\hline & T5 & $77-94$ & 338 \\
\hline & $\mathrm{T} 6$ & $94-120$ & 395 \\
\hline & $\mathrm{T} 7$ & $120-180$ & 303 \\
\hline & S1 & toluene & 966 \\
\hline & $\mathrm{S} 2$ & dichloromethane & 601 \\
\hline & S3 & tetrahydrofuran & 318 \\
\hline & S4 & 1,2-dichloro-ethane & 171 \\
\hline & S5 & 1,2-dimethoxyethane & 145 \\
\hline & S6 & acetonitrile & 141 \\
\hline & S7 & not listed & 102 \\
\hline
\end{tabular}


Table S4 - continued from previous page

\begin{tabular}{|c|c|c|c|}
\hline category & bin label & dataset name & instances \\
\hline \multirow{8}{*}{ solvent } & S8 & water & 71 \\
\hline & S9 & benzene & 76 \\
\hline & S10 & para-xylene & 136 \\
\hline & S11 & hexane & 43 \\
\hline & S12 & dimethyl sulfoxide & 39 \\
\hline & S13 & 1,4-dioxane & 33 \\
\hline & S14 & dibutyl ether & 33 \\
\hline & S15 & diethyl ether & 22 \\
\hline \multirow{11}{*}{ activator } & A1 & 4-methylmorpholine N-oxide & 420 \\
\hline & A2 & trimethylamine-N-oxide & 212 \\
\hline & A3 & dimethyl sulfoxide & 137 \\
\hline & A4 & cyclohexylamine & 68 \\
\hline & A5 & n-butyl methyl sulfide & 27 \\
\hline & A6 & silver trifluoromethanesulfonate & 23 \\
\hline & A7 & silver tetrafluoroborate & 18 \\
\hline & A8 & silver hexafluoroantimonate & 19 \\
\hline & A9 & (4-fluorobenzyl)(methyl)sulfide & 14 \\
\hline & A10 & dinitrogen monoxide & 14 \\
\hline & A11 & 4-methylmorpholine 4-oxide monohydrate & 13 \\
\hline \multirow{2}{*}{$\mathrm{CO}(\mathrm{g})$} & G1 & carbon monoxide & 1169 \\
\hline & G2 & none & 1580 \\
\hline \multirow{13}{*}{ additive } & O1 & 4 A molecular sieve & 84 \\
\hline & $\mathrm{O} 2$ & zinc & 50 \\
\hline & O3 & hydrogen & 40 \\
\hline & $\mathrm{O} 4$ & ethylene glycol & 30 \\
\hline & O5 & cetyltrimethylammonim bromide & 22 \\
\hline & O6 & Celite & 17 \\
\hline & O7 & Triton X(R)-100 & 37 \\
\hline & O8 & acetic anhydride & 15 \\
\hline & O9 & lithium chloride & 15 \\
\hline & $\mathrm{O} 10$ & water & 11 \\
\hline & O11 & oxygen & 10 \\
\hline & $\mathrm{O} 12$ & potassium carbonate & 8 \\
\hline & O13 & triethylsilane & 8 \\
\hline \multirow{4}{*}{ pressure } & P1 & $37-760$ & 35 \\
\hline & P2 & 760 & 2392 \\
\hline & P3 & $760-7600$ & 169 \\
\hline & $\mathrm{P} 4$ & $7600-7500600$ & 153 \\
\hline
\end{tabular}




\section{S2 Computational details and hyperparameters}

\section{S2.1 Gradient-boosting machines (GBMs)}

Numerical inputs for GBM models were constructed by tokenizing SMILES strings for each molecule in a reaction with character-to-number mappings, and calculating chemical descriptor vectors using Mordred. $\frac{[\mathbf{S 2}}{}$ Code examples for these processing protocols are provided in the associated github repository at the path data/gbm $\left\{_{-}\right\}$inputs/parsing-cols.ipynb.

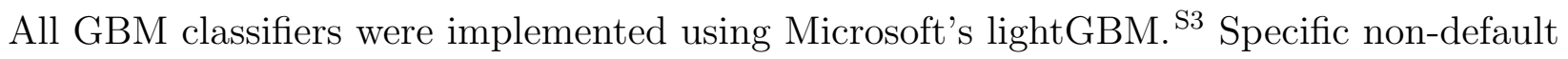
parameter settings are included in Table S5.

Table S5: Computational details and general parameters used for GBM models.

\begin{tabular}{|c|c|c|}
\hline parameter & value & description \\
\hline train/valid/test & $81 / 9 / 10$ & data splitting $^{a}$ \\
\hline max_depth & 7 & maximum tree depth for base learners \\
\hline $\begin{array}{l}\text { tree_method } \\
\text { eval_metric }\end{array}$ & $\begin{array}{l}\text { 'gpu_hist' } \\
\text { 'aucpr' }\end{array}$ & $\begin{array}{c}\text { split continuous features into discrete bins } \\
\text { evaluation metric }\end{array}$ \\
\hline
\end{tabular}

${ }^{a}$ Training, validation, and test sets were identical to those in GCNs.

\section{S2.1.1 Binary relevance method (BM)}

In BM experiments, an independent lightgbm.LGBMClassifier was fit for each label bin in a dataset's dictionary using the full input representation.

\section{S2.1.2 Classifier trellises (CTs)}

In CT experiments, lightgbm.LGBMClassifiers were fit for each label bin in a dataset's dictionary as part of a grid structure in which predictions are made sequentially and are passed to downstream models as additional inputs (see main text for explanation). Mutual information (MI) matrices were constructed for each dataset's label dictionary using scikit learn's sklearn.metrics.mutual_info_score module. $\frac{\mathrm{S4}}{}$ Classifier trellises were then constructed following the algorithm reported by Read et al. (see main text and associated

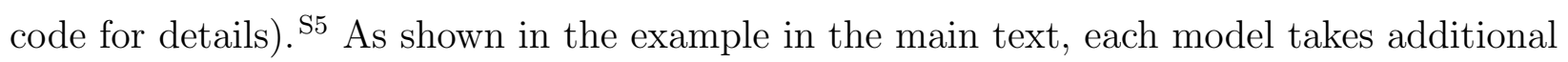


input from the bins in directions north, west, and northwest of it. Models on the edges of the trellis take input only from those bins in the available directions (i.e., propagation does not wrap between rows). Here each trellis was initialized using the label M1, the most commonly used metal in each dataset. This can be chosen by user preference, expert intuition, or at random. Full MI matrices and trellis structures for all four datasets are provided below. 


\begin{tabular}{|c|c|c|c|c|c|c|c|c|c|c|c|}
\hline Suzuki & 0 & 1 & 2 & 3 & 4 & 5 & 6 & 7 & 8 & 9 & 10 \\
\hline A & M1 & M_NULL & M2 & L_NULL & L1 & M3 & S4 & S7 & S2 & S1 & S5 \\
\hline B & M5 & M4 & B2 & B1 & B3 & L2 & S_NULL & S9 & S6 & L8 & B9 \\
\hline C & M6 & L3 & B4 & B_NULL & B5 & L4 & M13 & L22 & B6 & B15 & L6 \\
\hline D & L7 & L13 & M11 & B8 & A_NULL & A1 & M21 & B13 & L23 & M12 & M14 \\
\hline E & B26 & M8 & B14 & M27 & A3 & A4 & S3 & B28 & L12 & A11 & L9 \\
\hline $\mathbf{F}$ & L14 & L5 & A9 & B11 & B22 & B7 & M10 & B18 & B16 & L21 & B10 \\
\hline G & B25 & M9 & M7 & A17 & M16 & M24 & A6 & L19 & M19 & L20 & M18 \\
\hline $\mathbf{H}$ & M15 & B30 & B23 & B31 & L10 & A5 & B27 & B12 & M23 & S10 & B19 \\
\hline I & B35 & B21 & M17 & A14 & B20 & B17 & L15 & B24 & L11 & A8 & M20 \\
\hline J & L16 & M26 & A7 & L18 & A10 & A12 & L17 & A13 & B33 & M22 & A15 \\
\hline K & M25 & B29 & A16 & B32 & B34 & M28 & S8 & $A 2$ & None & None & None \\
\hline
\end{tabular}

Figure S1: Optimized classifier trellis for the Suzuki dataset.

\begin{tabular}{|c|c|c|c|c|c|c|c|c|c|c|c|}
\hline Suzuki & 0 & 1 & 2 & 3 & 4 & 5 & 6 & 7 & 8 & 9 & 10 \\
\hline A & 0 & 0.096509 & 0.022812 & 0.088898 & 0.069872 & 0.00271 & 0.014156 & 0.05331 & 0.052397 & 0.01702 & 0.013595 \\
\hline B & 0.032864 & 0.048547 & 0.024051 & 0.144962 & 0.071762 & 0.016851 & 0.025646 & 0.091712 & 0.012334 & 0.009231 & 0.006801 \\
\hline C & 0.002359 & 0.008967 & 0.031369 & 0.023736 & 0.01403 & 0.007808 & 0.005451 & 0.014139 & 0.003967 & 0.003043 & 0.00406 \\
\hline D & 0.00682 & 0.00365 & 0.002436 & 0.001583 & 0.002287 & 0.066936 & 0.000948 & 0.000183 & 0.000755 & 0.000702 & 0.000681 \\
\hline E & 0.001079 & 0.000182 & 0.000941 & 0.001686 & 0.018303 & 0.017265 & 0.001083 & 0.000846 & 0.000162 & 0.000657 & 0.000576 \\
\hline $\mathbf{F}$ & 4.60E-05 & 0.000387 & 0.000201 & 0.001154 & 0.000893 & 0.000775 & 0.000563 & 0.000979 & 0.000263 & $9.15 \mathrm{E}-05$ & 0.000275 \\
\hline G & 4.44E-05 & 0.000164 & 0.000112 & 0.000673 & 0.000912 & 0.000506 & 0.000619 & 0.001873 & 0.000835 & 0.000212 & 0.000182 \\
\hline $\mathbf{H}$ & 7.74E-06 & 0.001508 & 0.000412 & 0.000295 & $2.57 \mathrm{E}-05$ & 0.000116 & 0.000527 & $3.03 E-05$ & 0.000373 & $5.41 \mathrm{E}-05$ & 0.00013 \\
\hline I & 0.000791 & $9.31 \mathrm{E}-05$ & 4.80E-05 & $1.21 \mathrm{E}-05$ & 0.000433 & $2.48 \mathrm{E}-05$ & $6.91 \mathrm{E}-05$ & $3.25 \mathrm{E}-05$ & 3.70E-05 & $1.00 \mathrm{E}-05$ & 0.008369 \\
\hline J & $1.01 \mathrm{E}-06$ & 0.002866 & $5.53 \mathrm{E}-06$ & $5.72 E-06$ & $5.82 \mathrm{E}-06$ & 2.09E-05 & $6.06 \mathrm{E}-06$ & $3.68 \mathrm{E}-05$ & $6.95 \mathrm{E}-06$ & $5.40 \mathrm{E}-06$ & 6.35E-05 \\
\hline $\mathrm{K}$ & $1.45 \mathrm{E}-06$ & 0.008351 & $2.73 E-06$ & 3.12E-06 & $2.78 \mathrm{E}-06$ & 2.27E-06 & $1.78 \mathrm{E}-15$ & $3.55 \mathrm{E}-15$ & None & None & None \\
\hline
\end{tabular}

Figure S2: Mutual information matrix for the Suzuki dataset. 


\begin{tabular}{|c|c|c|c|c|c|c|c|c|c|c|c|c|c|c|c|}
\hline $\mathrm{C}-\mathrm{N}$ & 0 & 1 & 2 & 3 & 4 & 5 & 6 & 7 & 8 & 9 & 10 & 11 & 12 & 13 & 14 \\
\hline A & M1 & M_NULL & L_NULL & M2 & B1 & S1 & L1 & M3 & B3 & B2 & M4 & S13 & A7 & A_NULL & $\mathrm{A} 1$ \\
\hline B & B4 & B_NULL & S_NULL & L3 & L2 & S3 & S2 & S4 & L7 & B5 & S17 & A16 & $\mathrm{M} 8$ & A2 & S5 \\
\hline C & L16 & L4 & S10 & s6 & B8 & M5 & L24 & B6 & L31 & s7 & L26 & S12 & A13 & B9 & A19 \\
\hline D & A23 & M26 & M14 & L10 & M36 & L28 & S15 & M7 & A70 & M39 & A73 & M11 & A52 & A74 & M16 \\
\hline E & M6 & S9 & M23 & L35 & A69 & 58 & L5 & L15 & L8 & M12 & $\mathrm{M} 20$ & L18 & B12 & A49 & L19 \\
\hline $\mathbf{F}$ & A46 & A35 & L44 & B7 & A29 & L34 & A9 & L6 & A18 & S20 & M13 & A20 & A26 & L42 & M44 \\
\hline G & S11 & A67 & A11 & B11 & S16 & M33 & M10 & L29 & A40 & M15 & L23 & L9 & M24 & L25 & M21 \\
\hline H & L13 & A65 & L11 & M17 & S19 & L43 & L32 & L40 & M18 & A34 & M9 & A42 & L36 & S14 & M19 \\
\hline 1 & L12 & S18 & $\mathrm{M} 43$ & B10 & A17 & A56 & L14 & A62 & A22 & L17 & A72 & A43 & L20 & S21 & A10 \\
\hline $\mathrm{J}$ & M29 & M28 & A60 & L22 & A6 & L41 & M30 & L21 & A33 & A53 & S22 & A32 & B13 & A12 & A8 \\
\hline K & L27 & L30 & A15 & L33 & L46 & L38 & L37 & A21 & M22 & M25 & M27 & A24 & L39 & M31 & M34 \\
\hline $\mathbf{L}$ & M32 & M37 & A31 & A30 & M35 & M38 & A36 & M40 & A38 & M42 & L45 & A39 & A41 & M41 & A37 \\
\hline$M$ & A48 & A44 & A45 & A47 & A50 & A51 & A54 & A55 & L47 & A57 & A59 & A61 & A64 & A66 & A58 \\
\hline $\mathbf{N}$ & A63 & A68 & A71 & A3 & A4 & A5 & A14 & A25 & A27 & A28 & None & None & None & None & None \\
\hline
\end{tabular}

Figure S3: Optimized classifier trellis for the $\mathrm{C}-\mathrm{N}$ dataset.

\begin{tabular}{|c|c|c|c|c|c|c|c|c|c|c|c|c|c|c|c|}
\hline $\mathrm{C}-\mathrm{N}$ & 0 & 1 & 2 & 3 & 4 & 5 & 6 & 7 & 8 & 9 & 10 & 11 & 12 & 13 & 14 \\
\hline A & 0 & 0.073547 & 0.120096 & 0.083775 & 0.055793 & 0.114291 & 0.053129 & 0.025424 & 0.018588 & 0.046073 & 0.044959 & 0.021508 & 0.012579 & 0.008307 & 0.027749 \\
\hline B & 0.049414 & 0.055036 & 0.059134 & 0.074614 & 0.06015 & 0.078562 & 0.066955 & 0.036756 & 0.035659 & 0.027453 & 0.021502 & 0.003319 & 0.007042 & 0.023636 & 0.036117 \\
\hline C & 0.012605 & 0.013552 & 0.006146 & 0.004989 & 0.01415 & 0.012901 & 0.009452 & 0.009538 & 0.005913 & 0.005255 & 0.01089 & 0.000406 & 0.007037 & 0.006807 & 0.006894 \\
\hline D & 0.006856 & 0.005034 & 0.004996 & 0.005457 & 0.005872 & 0.007714 & 0.005609 & 0.005052 & 0.00504 & 0.002448 & 0.002415 & 0.001498 & 0.003485 & 0.003168 & 0.008106 \\
\hline $\mathbf{E}$ & $4.42 \mathrm{E}-05$ & 0.003866 & 0.005761 & 0.010098 & 0.004365 & 0.003708 & 0.013564 & 0.003323 & 0.000338 & 0.002307 & 0.002772 & 0.003058 & 0.000993 & 0.002919 & 0.004763 \\
\hline $\mathbf{F}$ & 0.003656 & 0.00644 & 0.004916 & 0.005547 & 0.004101 & 0.005527 & 0.020156 & 0.000646 & 0.002133 & 0.000985 & 0.00025 & 0.00552 & 0.002737 & $1.00 \mathrm{E}-04$ & 0.00187 \\
\hline G & $1.25 \mathrm{E}-05$ & 0.003128 & 0.002674 & 0.000967 & 0.003233 & 0.004753 & $8.59 \mathrm{E}-05$ & 0.013501 & 0.001922 & 0.003306 & 0.00346 & 0.000136 & 0.000183 & 0.000745 & $8.71 \mathrm{E}-05$ \\
\hline H & 0.001328 & 0.001261 & 0.001202 & 0.001433 & 0.003342 & 0.000258 & 0.010713 & 0.007045 & 0.000557 & 0.001991 & 7.92E-05 & 0.004235 & 0.000586 & $6.64 \mathrm{E}-05$ & 0.000463 \\
\hline 1 & 4.80E-05 & 0.002084 & 0.004955 & 0.000705 & 0.005093 & 0.005229 & 2.99E-05 & 0.002696 & $7.90 E-05$ & 3.93E-05 & 0.00057 & 0.00039 & 2.02E-05 & $7.13 \mathrm{E}-05$ & 0.004068 \\
\hline J & 0.001079 & 0.002274 & 0.000352 & 3.68E-05 & $6.62 E-05$ & 0.004622 & 0.000946 & $3.95 \mathrm{E}-05$ & 0.000296 & 0.000111 & 0.000229 & 0.004983 & $2.51 \mathrm{E}-05$ & 7.02E-05 & $3.75 E-05$ \\
\hline K & 8.14E-06 & $1.85 \mathrm{E}-05$ & 1.19E-05 & $1.94 \mathrm{E}-05$ & 0.000124 & 1.44E-05 & $1.01 \mathrm{E}-05$ & $1.49 \mathrm{E}-05$ & $1.35 E-05$ & $5.68 \mathrm{E}-06$ & $9.68 \mathrm{E}-06$ & $1.00 E-05$ & 8.89E-06 & $1.12 \mathrm{E}-05$ & 0.004299 \\
\hline $\mathbf{L}$ & $4.26 \mathrm{E}-06$ & $9.19 \mathrm{E}-06$ & $8.02 \mathrm{E}-06$ & 7.56E-06 & $5.26 \mathrm{E}-06$ & $4.38 \mathrm{E}-06$ & $5.58 \mathrm{E}-06$ & $5.23 \mathrm{E}-06$ & $5.00 E-06$ & 4.50E-06 & 4.13E-06 & $3.94 \mathrm{E}-06$ & $3.88 \mathrm{E}-06$ & $3.67 \mathrm{E}-06$ & $3.43 E-06$ \\
\hline$M$ & $1.33 E-06$ & $3.38 \mathrm{E}-06$ & $3.21 \mathrm{E}-06$ & $3.12 \mathrm{E}-06$ & $3.06 \mathrm{E}-06$ & $2.75 \mathrm{E}-06$ & $2.62 \mathrm{E}-06$ & $2.50 \mathrm{E}-06$ & $2.36 \mathrm{E}-06$ & $2.31 \mathrm{E}-06$ & 2.19E-06 & $2.15 \mathrm{E}-06$ & 2.04E-06 & $1.91 \mathrm{E}-06$ & $1.78 \mathrm{E}-06$ \\
\hline $\mathbf{N}$ & $6.21 \mathrm{E}-07$ & $1.56 \mathrm{E}-06$ & $1.45 \mathrm{E}-06$ & 3.47E-18 & $3.47 E-18$ & $3.47 E-18$ & 3.47E-18 & $1.78 \mathrm{E}-15$ & $1.77 \mathrm{E}-15$ & 0 & None & None & None & None & None \\
\hline
\end{tabular}

Figure S4: Mutual information matrix for the $\mathrm{C}-\mathrm{N}$ dataset. 


\begin{tabular}{|c|c|c|c|c|c|c|c|c|c|c|c|}
\hline Negishi & 0 & 1 & 2 & 3 & 4 & 5 & 6 & 7 & 8 & 9 & 10 \\
\hline A & M1 & L_NULL & M2 & L1 & $\mathrm{A} 2$ & A_NULL & $\mathrm{A} 1$ & $\mathrm{~A} 3$ & M_NULL & 01 & O_NULL \\
\hline B & S3 & M9 & L3 & S1 & S2 & T8 & T2 & $\mathrm{T} 4$ & T3 & S_NULL & L17 \\
\hline C & T7 & M12 & S5 & T5 & T6 & S6 & M3 & M10 & A10 & A11 & M16 \\
\hline D & M4 & L2 & M6 & M14 & L12 & M29 & L15 & S4 & M26 & M5 & L5 \\
\hline E & A16 & A13 & S10 & L4 & M18 & M7 & M8 & $\mathrm{M} 27$ & A19 & L6 & S8 \\
\hline $\mathbf{F}$ & S7 & A8 & M15 & L10 & $\mathrm{M} 22$ & A7 & A4 & L11 & M11 & S9 & A12 \\
\hline G & L19 & A5 & A21 & M21 & A24 & L8 & M19 & A17 & $\mathrm{A} 20$ & A14 & A15 \\
\hline H & T1 & M13 & A25 & L20 & L7 & M17 & M20 & A9 & M23 & M24 & A22 \\
\hline I & M30 & L9 & M25 & L13 & L14 & M28 & M32 & A18 & M31 & L16 & L18 \\
\hline J & A23 & A26 & A6 & A28 & A29 & A30 & A27 & T_NULL & None & None & None \\
\hline
\end{tabular}

Figure S5: Optimized classifier trellis for the Negishi dataset.

\begin{tabular}{|c|c|c|c|c|c|c|c|c|c|c|c|}
\hline Negishi & 0 & 1 & 2 & 3 & 4 & 5 & 6 & 7 & 8 & 9 & 10 \\
\hline A & 0 & 0.084283 & 0.085225 & 0.044055 & 0.030646 & 0.063871 & 0.072934 & 0.025056 & 0.011172 & 0.082987 & 0.437132 \\
\hline B & 0.030077 & 0.082105 & 0.082442 & 0.039851 & 0.154657 & 0.050113 & 0.0354 & 0.070173 & 0.024463 & 0.036936 & 0.025029 \\
\hline C & 0.062908 & 0.066378 & 0.047095 & 0.028718 & 0.043761 & 0.014863 & 0.025536 & 0.015061 & 0.014118 & 0.05371 & 0.025252 \\
\hline D & 0.003846 & 0.059757 & 0.019728 & 0.015168 & 0.036481 & 0.01511 & 0.010263 & 0.009652 & 0.013173 & 0.00102 & 0.039501 \\
\hline E & 0.014352 & 0.041992 & 0.02248 & 0.013086 & 0.016298 & 0.000712 & 0.00201 & 0.010624 & 0.006797 & 0.023566 & 0.011486 \\
\hline $\mathbf{F}$ & 0.000134 & 0.009139 & 0.005586 & 0.029858 & 0.002824 & 0.001286 & 0.016869 & 0.006107 & 0.000223 & 0.000564 & 0.010772 \\
\hline G & 0.005944 & 0.001132 & 0.000382 & 0.006866 & 0.000311 & 0.011533 & 0.000385 & 0.000847 & 0.000372 & 0.009572 & 0.002582 \\
\hline H & $3.18 \mathrm{E}-05$ & 0.000215 & 0.012096 & 0.017011 & $7.66 \mathrm{E}-05$ & 0.000149 & 0.000209 & 0.042064 & $8.24 \mathrm{E}-05$ & $7.84 \mathrm{E}-05$ & 0.018103 \\
\hline I & $8.86 \mathrm{E}-05$ & 0.000151 & $9.11 \mathrm{E}-05$ & $5.23 \mathrm{E}-05$ & 0.002081 & $8.62 \mathrm{E}-05$ & $7.51 \mathrm{E}-05$ & $6.89 \mathrm{E}-05$ & 5.97E-05 & $5.38 \mathrm{E}-05$ & 4.30E-05 \\
\hline J & 1.39E-05 & 3.35E-05 & $2.63 \mathrm{E}-05$ & $1.98 \mathrm{E}-05$ & 1.71E-05 & 0.012082 & $3.54 \mathrm{E}-15$ & $3.54 \mathrm{E}-15$ & None & None & None \\
\hline
\end{tabular}

Figure S6: Mutual information matrix for the Negishi dataset. 


\begin{tabular}{|c|c|c|c|c|c|c|c|c|c|c|}
\hline PKR & $\mathbf{0}$ & $\mathbf{1}$ & $\mathbf{2}$ & $\mathbf{3}$ & $\mathbf{4}$ & $\mathbf{5}$ & $\mathbf{6}$ & $\mathbf{7}$ & $\mathbf{8}$ & $\mathbf{9}$ \\
\hline A & M1 & M_NULL & G1 & G_NULL & A_NULL & A1 & S2 & S1 & S3 & M6 \\
\hline B & M2 & S10 & P2 & P3 & A2 & T2 & T6 & S4 & T5 & T3 \\
\hline C & O_NULL & O1 & P4 & T7 & M12 & T4 & L1 & L_NULL & L2 & M3 \\
\hline D & O2 & M5 & M13 & S14 & O8 & S5 & A4 & S9 & L5 & S_NULL \\
\hline E & S6 & A10 & N1 & N_NULL & L3 & S13 & M9 & O7 & S8 & O5 \\
\hline F & O10 & T1 & O3 & A3 & P1 & O4 & S7 & M11 & O6 & M4 \\
\hline G & M8 & M17 & A8 & M14 & S11 & O11 & S12 & M10 & M7 & A7 \\
\hline H & A5 & A6 & L6 & S15 & L4 & M16 & O9 & A9 & M15 & 012 \\
\hline I & O13 & M18 & A11 & T_NULL & P_NULL & None & None & None & None & None \\
\hline
\end{tabular}

Figure S7: Optimized classifier trellis for the PKR dataset.

\begin{tabular}{|c|c|c|c|c|c|c|c|c|c|c|}
\hline PKR & $\mathbf{0}$ & $\mathbf{1}$ & $\mathbf{2}$ & $\mathbf{3}$ & $\mathbf{4}$ & $\mathbf{5}$ & $\mathbf{6}$ & $\mathbf{7}$ & $\mathbf{8}$ & $\mathbf{9}$ \\
\hline A & 0 & 0.148624 & 0.160515 & 0.68243 & 0.102405 & 0.186185 & 0.131708 & 0.071628 & 0.049108 & 0.023795 \\
\hline B & 0.035102 & 0.053809 & 0.084487 & 0.233286 & 0.135261 & 0.170539 & 0.104031 & 0.05365 & 0.039864 & 0.047309 \\
\hline C & 0.018397 & 0.07792 & 0.133582 & 0.086451 & 0.039753 & 0.070014 & 0.051156 & 0.118731 & 0.084137 & 0.072301 \\
\hline D & 0.041782 & 0.115446 & 0.008828 & 0.028101 & 0.040167 & 0.02588 & 0.086418 & 0.043118 & 0.03207 & 0.0205 \\
\hline E & 0.001884 & 0.015424 & 0.002866 & 0.453932 & 0.013475 & 0.019953 & 0.015342 & 0.024111 & 0.008457 & 0.032315 \\
\hline F & 0.007421 & 0.006052 & 0.012843 & 0.012669 & 0.009257 & 0.007902 & 0.005033 & 0.022851 & 0.023849 & 0.001589 \\
\hline G & $8.69 \mathrm{E}-05$ & 0.006557 & 0.001302 & 0.022536 & 0.001213 & 0.002937 & 0.000731 & 0.029565 & 0.000277 & 0.003329 \\
\hline H & 0.000182 & 0.000289 & 0.026024 & 0.00011 & 0.000167 & 0.00096 & 0.000128 & 0.000159 & 0.000113 & $7.75 \mathrm{E}-05$ \\
\hline I & $2.94 \mathrm{E}-05$ & $4.65 \mathrm{E}-05$ & $2.08 \mathrm{E}-05$ & $1.77 \mathrm{E}-15$ & $8.82 \mathrm{E}-16$ & None & None & None & None & None \\
\hline
\end{tabular}

Figure S8: Mutual information matrix for the PKR dataset. 


\section{S2.2 Graph convolutional networks (GCNs)}

Molecular graph calculations and all neural network (NN) architectures tested herein were implemented using the Chainer Chemistry (ChainerChem) library $\sqrt{\mathbf{S} 6}$. Our previous study

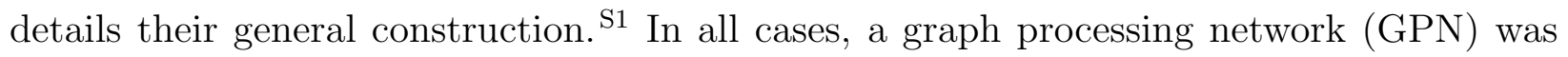
constructed and combined with a dense multi-layer perceptron (MLP), which were trained together as a joint network. All models were trained for 100 epochs on 1 NVIDIA K80 GPU device, unless otherwise specified. Training and test sets were held consistent between models for each reaction dataset. This was done by first splitting each dataset into 90/10 train/test, then splitting the training set into $90 / 10$ train/validation, resulting in a final split of $81 / 9 / 10$ train/validation/test overall. A dummy predictor that always predicts the most frequent bin in each label category was also created for each dataset as a baseline performance reference.

General parameters and hyperparameter settings are summarized in Table S6, which are held constant between R-GCN and AR-GCN models for all datasets.

Table S6: Computational details and general parameters used for GCN models.

\begin{tabular}{c|c|c}
\hline parameter & value & description \\
\hline loss & sigmoid cross entropy & loss function used for training \\
optimizer & Adam & model optimization algorithm \\
train/valid/test & $81 / 9 / 10$ & data splitting \\
batch size & 32 & batch size used for gradient calculations \\
epochs & 100 & number of training epochs \\
out_dim & 128 & number of units in the readout \\
hidden_dim & 128 & number of units in the hidden layers \\
n_layers & 4 & number of convolutional layers \\
n_atom_types & 117 & number of allowed atom types \\
concat_hidden & False & readouts concatenated at each layer \\
ch_list & None & channels in update layers \\
input_type & 'int' & input vector type \\
scale_adj & True & normalize adjacency matrix \\
\hline
\end{tabular}

${ }^{a}$ AR-GCNs have two additional attention layers with hidden_dim=128 and out_dim= 128. 


\section{S3 Expanded results}

\section{S3.1 Average accuracy}

Expanded modeling results separating accuracies $\left(\mathrm{A}_{c}\right)$ and error reductions $(\mathrm{ER})$ are included in Tables $\mathrm{S} 7 \mathrm{H} 10$ along with their averages $\mathrm{AA}_{c}$ and $\mathrm{AER}$, respectively (see main text for equations describing their calculation). It should be noted that since the "CO (g)" category in the PKR dataset is a binary class (either yes or no), the top-3 accuracy will always be 1 . This category is therefore excluded from AER and $\mathrm{AA}_{c}$ calculations for this section. 
Table S7: Top-1 $\mathrm{A}_{c}$ and $\mathrm{AA}_{c}$ for all model types on all four datasets.

\begin{tabular}{|c|c|c|c|c|c|c|}
\hline dataset & category & dummy & BM-GBM & CT-GBM & $\mathrm{R}-\mathrm{GCN}$ & AR-GCN \\
\hline \multirow{6}{*}{ Suzuki } & $\boldsymbol{A} \boldsymbol{A}_{c}$ & 0.6350 & 0.7004 & 0.6952 & 0.7494 & 0.7663 \\
\hline & metal & 0.3777 & 0.5732 & 0.5629 & 0.6306 & 0.6499 \\
\hline & ligand & 0.8722 & 0.8390 & 0.8408 & 0.9036 & 0.9081 \\
\hline & base & 0.3361 & 0.4908 & 0.4777 & 0.5455 & 0.5896 \\
\hline & solvent & 0.6377 & 0.6729 & 0.6751 & 0.7049 & 0.7217 \\
\hline & additive & 0.9511 & 0.9259 & 0.9196 & 0.9624 & 0.9621 \\
\hline \multirow{6}{*}{$\mathrm{C}-\mathrm{N}$} & $\boldsymbol{A} \boldsymbol{A}_{c}$ & 0.4455 & 0.5528 & 0.5370 & 0.6706 & 0.6793 \\
\hline & metal & 0.2452 & 0.4825 & 0.4582 & 0.5989 & 0.6162 \\
\hline & ligand & 0.5219 & 0.5538 & 0.5710 & 0.6981 & 0.7068 \\
\hline & base & 0.2479 & 0.5028 & 0.5003 & 0.5932 & 0.6066 \\
\hline & solvent & 0.3219 & 0.4582 & 0.4524 & 0.5647 & 0.5674 \\
\hline & additive & 0.8904 & 0.7669 & 0.7031 & 0.8984 & 0.8997 \\
\hline \multirow{6}{*}{ Negishi } & $\boldsymbol{A} \boldsymbol{A}_{c}$ & 0.5866 & 0.7506 & 0.7312 & 0.7812 & 0.7914 \\
\hline & metal & 0.2887 & 0.5444 & 0.5218 & 0.6555 & 0.6730 \\
\hline & ligand & 0.7879 & 0.8174 & 0.7900 & 0.8724 & 0.8772 \\
\hline & temperature & 0.3317 & 0.6656 & 0.6527 & 0.6188 & 0.6507 \\
\hline & solvent & 0.6938 & 0.8562 & 0.8514 & 0.8868 & 0.8915 \\
\hline & additive & 0.8309 & 0.8691 & 0.8401 & 0.8724 & 0.8644 \\
\hline \multirow{9}{*}{ PKR } & $\boldsymbol{A} \boldsymbol{A}_{c}$ & 0.6123 & 0.8010 & 0.7925 & 0.8005 & 0.8101 \\
\hline & metal & 0.4302 & 0.7901 & 0.7786 & 0.7132 & 0.7057 \\
\hline & ligand & 0.8792 & 0.9351 & 0.9237 & 0.9057 & 0.9094 \\
\hline & temperature & 0.2830 & 0.5954 & 0.5649 & 0.6528 & 0.6642 \\
\hline & solvent & 0.3321 & 0.6183 & 0.6260 & 0.6792 & 0.6981 \\
\hline & activator & 0.6906 & 0.8244 & 0.8015 & 0.8415 & 0.8491 \\
\hline & $\mathrm{CO}(\mathrm{g})$ & 0.7245 & 0.8855 & 0.8855 & 0.8717 & 0.8868 \\
\hline & additive & 0.9057 & 0.9008 & 0.8893 & 0.8906 & 0.8491 \\
\hline & pressure & 0.6528 & 0.8588 & 0.8702 & 0.8491 & 0.8491 \\
\hline
\end{tabular}


Table S8: Top-3 $\mathrm{A}_{c}$ and $\mathrm{AA}_{c}$ for all model types on all four datasets.

\begin{tabular}{|c|c|c|c|c|c|c|}
\hline dataset & category & dummy & BM-GBM & CT-GBM & R-GCN & AR-GCN \\
\hline \multirow{6}{*}{ Suzuki } & $\boldsymbol{A} \boldsymbol{A}_{c}$ & 0.8228 & 0.8994 & 0.8949 & 0.9004 & 0.9077 \\
\hline & metal & 0.6744 & 0.8516 & 0.8475 & 0.8482 & 0.8597 \\
\hline & ligand & 0.9269 & 0.9635 & 0.9606 & 0.9644 & 0.9676 \\
\hline & base & 0.7344 & 0.8338 & 0.8250 & 0.8123 & 0.8285 \\
\hline & solvent & 0.8013 & 0.8637 & 0.8577 & 0.8836 & 0.8897 \\
\hline & additive & 0.9771 & 0.9842 & 0.9832 & 0.9934 & 0.9931 \\
\hline \multirow{6}{*}{$\mathrm{C}-\mathrm{N}$} & $\boldsymbol{A} \boldsymbol{A}_{c}$ & 0.6881 & 0.8106 & 0.8016 & 0.8609 & 0.8621 \\
\hline & metal & 0.6526 & 0.7928 & 0.7772 & 0.8479 & 0.8490 \\
\hline & ligand & 0.6647 & 0.7933 & 0.7928 & 0.8605 & 0.8688 \\
\hline & base & 0.6400 & 0.8008 & 0.7916 & 0.8452 & 0.8370 \\
\hline & solvent & 0.5677 & 0.7370 & 0.7281 & 0.7973 & 0.7997 \\
\hline & additive & 0.9156 & 0.9290 & 0.9184 & 0.9534 & 0.9559 \\
\hline \multirow{6}{*}{ Negishi } & $\boldsymbol{A} \boldsymbol{A}_{c}$ & 0.7455 & 0.9044 & 0.8905 & 0.9085 & 0.9209 \\
\hline & metal & 0.5008 & 0.7771 & 0.7674 & 0.8086 & 0.8517 \\
\hline & ligand & 0.8549 & 0.9548 & 0.9321 & 0.9522 & 0.9553 \\
\hline & temperature & 0.5885 & 0.9031 & 0.8772 & 0.8517 & 0.8708 \\
\hline & solvent & 0.8788 & 0.9321 & 0.9402 & 0.9537 & 0.9537 \\
\hline & additive & 0.9043 & 0.9548 & 0.9354 & 0.9761 & 0.9729 \\
\hline \multirow{9}{*}{ PKR } & $\boldsymbol{A} \boldsymbol{A}_{c}{ }^{a}$ & 0.7973 & 0.9364 & 0.9302 & 0.9348 & 0.9402 \\
\hline & metal & 0.7132 & 0.9351 & 0.9313 & 0.9057 & 0.8906 \\
\hline & ligand & 0.9019 & 0.9962 & 0.9924 & 0.9849 & 0.9962 \\
\hline & temperature & 0.5962 & 0.8740 & 0.8321 & 0.8528 & 0.8604 \\
\hline & solvent & 0.5925 & 0.8779 & 0.8550 & 0.8679 & 0.8981 \\
\hline & activator & 0.8830 & 0.9466 & 0.9275 & 0.9774 & 0.9774 \\
\hline & $\mathrm{CO}(\mathrm{g})$ & 1.0000 & 1.0000 & 1.0000 & 1.0000 & 1.0000 \\
\hline & additive & 0.9321 & 0.9885 & 0.9885 & 0.9698 & 0.9736 \\
\hline & pressure & 0.9623 & 0.9771 & 0.9847 & 0.9849 & 0.9849 \\
\hline
\end{tabular}

${ }^{a}$ Excludes $C O(g)$. 


\section{S3.2 Error reduction}

Table S9: Top-1 ER and AER for all model types on all four datasets.

\begin{tabular}{|c|c|c|c|c|c|c|}
\hline dataset & category & dummy & BM-GBM & CT-GBM & R-GCN & AR-GCN \\
\hline \multirow{6}{*}{ Suzuki } & AER & - & $-0.0263^{a}$ & $-0.0554^{b}$ & 0.2767 & 0.3115 \\
\hline & metal & - & 0.3142 & 0.2977 & 0.4064 & 0.4374 \\
\hline & ligand & - & -0.2595 & -0.2455 & 0.2462 & 0.2809 \\
\hline & base & - & 0.2331 & 0.2134 & 0.3155 & 2934 \\
\hline & solvent & - & 0.0972 & 0.1032 & 0.1854 & 0.2319 \\
\hline & additive & - & -0.5164 & -0.6456 & 0.2298 & 0.2255 \\
\hline \multirow{6}{*}{$\mathrm{C}-\mathrm{N}$} & AER & - & $-0.0413^{c}$ & $-0.1593^{d}$ & 0.3453 & 0.3604 \\
\hline & metal & - & 0.3143 & 0.2822 & 0.4686 & 0.4915 \\
\hline & ligand & - & 0.0666 & 0.1027 & 0.3685 & 0.3868 \\
\hline & base & - & 0.3389 & 0.3355 & 0.4590 & 0.4769 \\
\hline & solvent & - & 0.2010 & 0.1924 & 0.3580 & 0.3620 \\
\hline & additive & - & -1.1275 & -1.7095 & 0.0725 & 0.0850 \\
\hline \multirow{6}{*}{ Negishi } & AER & - & 0.3510 & 0.2773 & 0.4439 & 0.4565 \\
\hline & metal & - & 0.3595 & 0.3277 & 0.5157 & 0.5404 \\
\hline & ligand & - & 0.1394 & 0.0099 & 0.3985 & 0.4211 \\
\hline & temperature & - & 0.4996 & 0.4802 & 0.4296 & 0.4773 \\
\hline & solvent & - & 0.5305 & 0.5146 & 0.6302 & 0.6458 \\
\hline & additive & - & 0.2260 & 0.0540 & 0.2453 & 0.1981 \\
\hline \multirow{9}{*}{ PKR } & AER & - & 0.4396 & 0.4010 & 0.3973 & 0.4199 \\
\hline & metal & - & 0.6316 & 0.6115 & 0.4967 & 0.4834 \\
\hline & ligand & - & 0.4627 & 0.3678 & 0.2188 & 0.2500 \\
\hline & temperature & - & 0.4357 & 0.3931 & 0.5158 & 0.5316 \\
\hline & solvent & - & 0.4286 & 0.4400 & 0.5198 & 0.5480 \\
\hline & activator & - & 0.4326 & 0.3586 & 0.4878 & 0.5122 \\
\hline & $\mathrm{CO}(\mathrm{g})$ & - & 0.5843 & 0.5843 & 0.5342 & 0.5890 \\
\hline & additive & - & -0.0519 & -0.1733 & -0.1600 & -0.1200 \\
\hline & pressure & - & 0.5932 & 0.6262 & 0.5652 & 0.5652 \\
\hline
\end{tabular}

AER excluding additive: ${ }^{a} 0.0962 .{ }^{b} 0.0922 .{ }^{c} 0.2302 .{ }^{d} 0.2282$. 
Table S10: Top-3 ER and AER for all model types on all four datasets.

\begin{tabular}{|c|c|c|c|c|c|c|}
\hline dataset & category & dummy & BM-GBM & CT-GBM & R-GCN & AR-GCN \\
\hline \multirow{6}{*}{ Suzuki } & AER & - & 0.4088 & 0.3774 & 0.4936 & 0.5246 \\
\hline & metal & - & 0.5442 & 0.5315 & 0.5336 & 0.5692 \\
\hline & ligand & - & 0.5013 & 0.4608 & 0.5137 & 0.5564 \\
\hline & base & - & 0.3741 & 0.3409 & 0.2934 & 0.3545 \\
\hline & solvent & - & 0.3140 & 0.2838 & 0.4142 & 0.4449 \\
\hline & additive & - & 0.3106 & 0.2698 & 0.7130 & 0.6979 \\
\hline \multirow{6}{*}{$\mathrm{C}-\mathrm{N}$} & AER & - & 0.3568 & 0.3131 & 0.5391 & 0.5471 \\
\hline & metal & - & 0.4034 & 0.3585 & 0.5623 & 0.5655 \\
\hline & ligand & - & 0.3837 & 0.3820 & 0.5842 & 0.6087 \\
\hline & base & - & 0.4468 & 0.4212 & 0.5700 & 0.5472 \\
\hline & solvent & - & 0.3918 & 0.3712 & 0.5311 & 0.5368 \\
\hline & additive & - & 0.1582 & 0.0328 & 0.4481 & 0.4773 \\
\hline \multirow{6}{*}{ Negishi } & AER & - & 0.5947 & 0.5199 & 0.6590 & 0.6833 \\
\hline & metal & - & 0.5534 & 0.5340 & 0.6166 & 0.7029 \\
\hline & ligand & - & 0.6883 & 0.5325 & 0.6703 & 0.6923 \\
\hline & temperature & - & 0.7644 & 0.7016 & 0.6395 & 0.6860 \\
\hline & solvent & - & 0.4402 & 0.5069 & 0.6184 & 0.6184 \\
\hline & additive & - & 0.5273 & 0.3247 & 0.7500 & 0.7167 \\
\hline \multirow{9}{*}{ PKR } & $\mathbf{A E R}^{a}$ & - & 0.6987 & 0.6740 & 0.6844 & 0.7145 \\
\hline & metal & - & 0.7738 & 0.7604 & 0.6711 & 0.6184 \\
\hline & ligand & - & 0.9611 & 0.9222 & 0.8462 & 0.9615 \\
\hline & temperature & - & 0.6881 & 0.5841 & 0.6355 & 0.6542 \\
\hline & solvent & - & 0.7003 & 0.6441 & 0.6759 & 0.7500 \\
\hline & activator & - & 0.5432 & 0.3801 & 0.8065 & 0.8065 \\
\hline & $\mathrm{CO}(\mathrm{g})$ & - & 1.0000 & 1.0000 & 1.0000 & 1.0000 \\
\hline & additive & - & 0.8314 & 0.8314 & 0.5556 & 0.6111 \\
\hline & pressure & - & 0.3931 & 0.5954 & 0.6000 & 0.6000 \\
\hline
\end{tabular}

${ }^{a}$ Excludes $C O(g)$ 


\section{S4 Adversarial controls}

Several control studies were conducted to ensure the validity of chemical feature learning. $\underline{\text { S7 }}$ The main text and results tables above describe the reference model known as the "dummy" predictor. This model simply returns the most frequently occurring top- $k$ labels in each dataset category as its prediction for each task. This gives a baseline accuracy to compare the actual learning our models achieve beyond simply fitting the dictionary frequency distributions. Additional controls conducted for the GBM models included:

1. Shuffling full inputs, leaving outputs in place (shuffle inputs)

2. Ablating the SMILES region of the inputs (Mordred only)

3. Substituting Mordred vectors with random unique numerical vectors (random Mordred)

4. Shuffling Mordred inputs, leaving outputs in place (shuffle Mordred)

5. Ablating the descriptor regions of the inputs (SMILES only)

6. Substituting SMILES vectors with random unique numerical vectors (random SMILES)

7. Shuffling SMILES inputs, leaving outputs in place (shuffle SMILES)

Each of these control models are compared to the standard BM-GBM model as well as the dummy predictor for all four datasets in Tables $\mathrm{S11}-\mathrm{S} 14$.

The results of these studies have very important implications, as they indicate that SMILES tokens are insufficient chemical representations for structure learning. On its own, that the SMILES-only models perform similarly to the hybrid (full input, BM-GBM) models in many cases could simply indicate the reduced dimensionality (300 vs 2304) helps feature learning and/or prevents underfitting. However, since some of the random SMILES control models retain similar accuracy, it must be concluded that little chemical feature learning is taking place in the SMILES models. Rather, SMILES are likely acting as unique "barcodes"

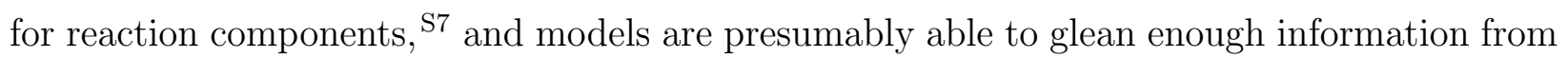


the presence of specific molecules in reactions to establish some accuracy greater than baseline. This is perhaps unsurprising in datasets such as the Negishi, where the chemical space is limited by dataset size and less-accessible organometallic nucleophiles than in datasets like Suzuki, C-N, and PKR. This results in fewer unique molecules and thus the ability to fit models to their presence or absence.

We strongly recommend caution in reaction learning when using SMILES representations. In line with recent commentary highlighting these control experiments, $\stackrel{\text { S7/S8 }}{ }$ we do not suggest that SMILES modeling is entirely futile, but that in our case models trained on SMILES only will likely be unable to generalize to reactions containing new molecules not in the current dataset. That said, these models could perhaps be used to predict reaction conditions with high accuracy for yet untested substrate pairs whose individual components have been previously reported. With the goal of creating generalizable models, we do not explore SMILES-only models any further.

On the other hand, the full-input and Mordred-only models pass the described adversarial controls in most all cases, leading to the conclusion that these input types are capable of representing our chemical space. Replacing the hybrid or Mordred representations with randomized vectors led to a large breakdown of model accuracy (random inputs and random Mordred), as did shuffling their input-output pairs (shuffle inputs and shuffle Mordred). To aid in interpreting these results, we compare AERs between the control models ("straw", $\mathrm{AER}_{s}$ ) and their featurized reference models ("feature", $\mathrm{AER}_{f}$ ). We calculate the difference in AER between these two models, and take the ratio of this difference to the reference model's AER to give $\mathrm{AER}_{d}$, which allows for comparison between model types as below. This translates to measuring the percent change in AER observed when true chemical features are replaced with random variables, and thus significant negative numbers should be expected.

$$
\mathrm{AER}_{d}=\frac{\mathrm{AER}_{s}-\mathrm{AER}_{f}}{\mathrm{AER}_{f}} \times 100 \%
$$




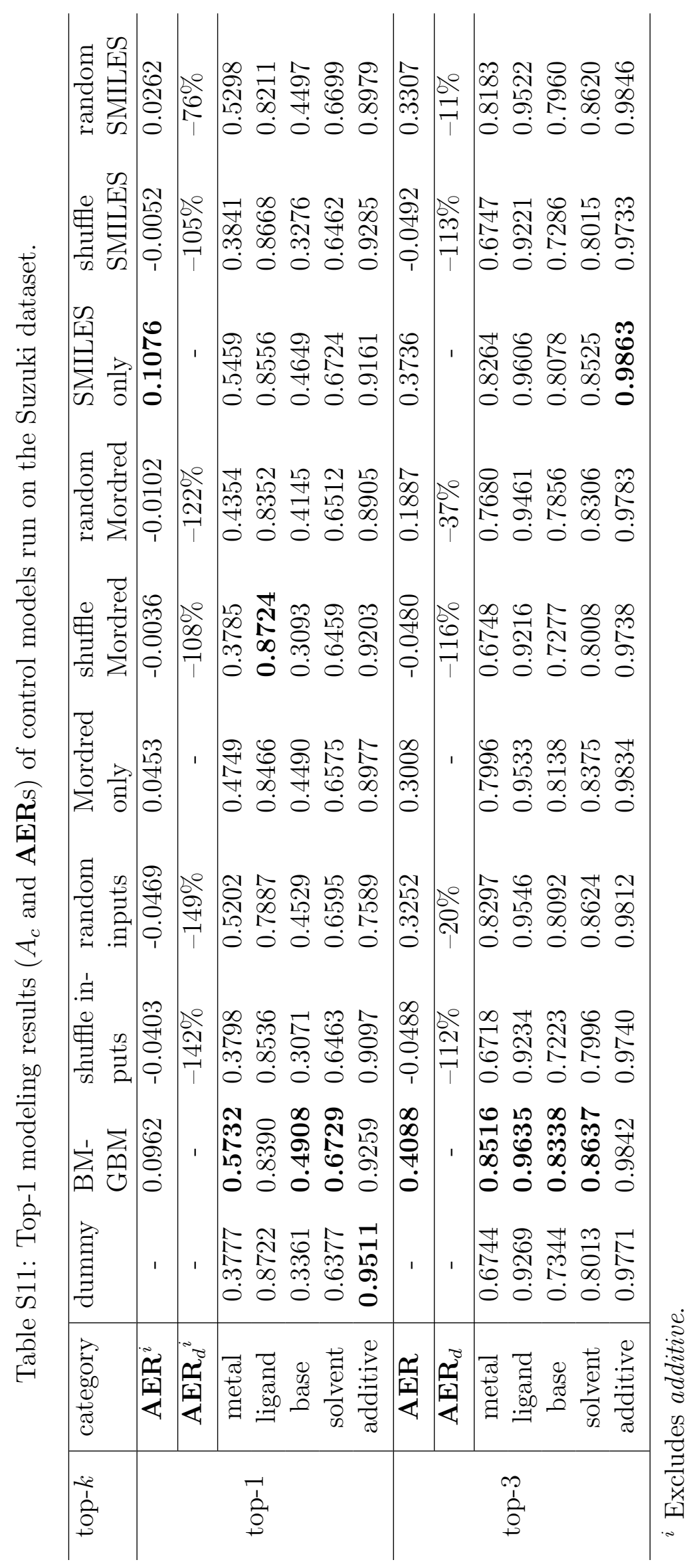




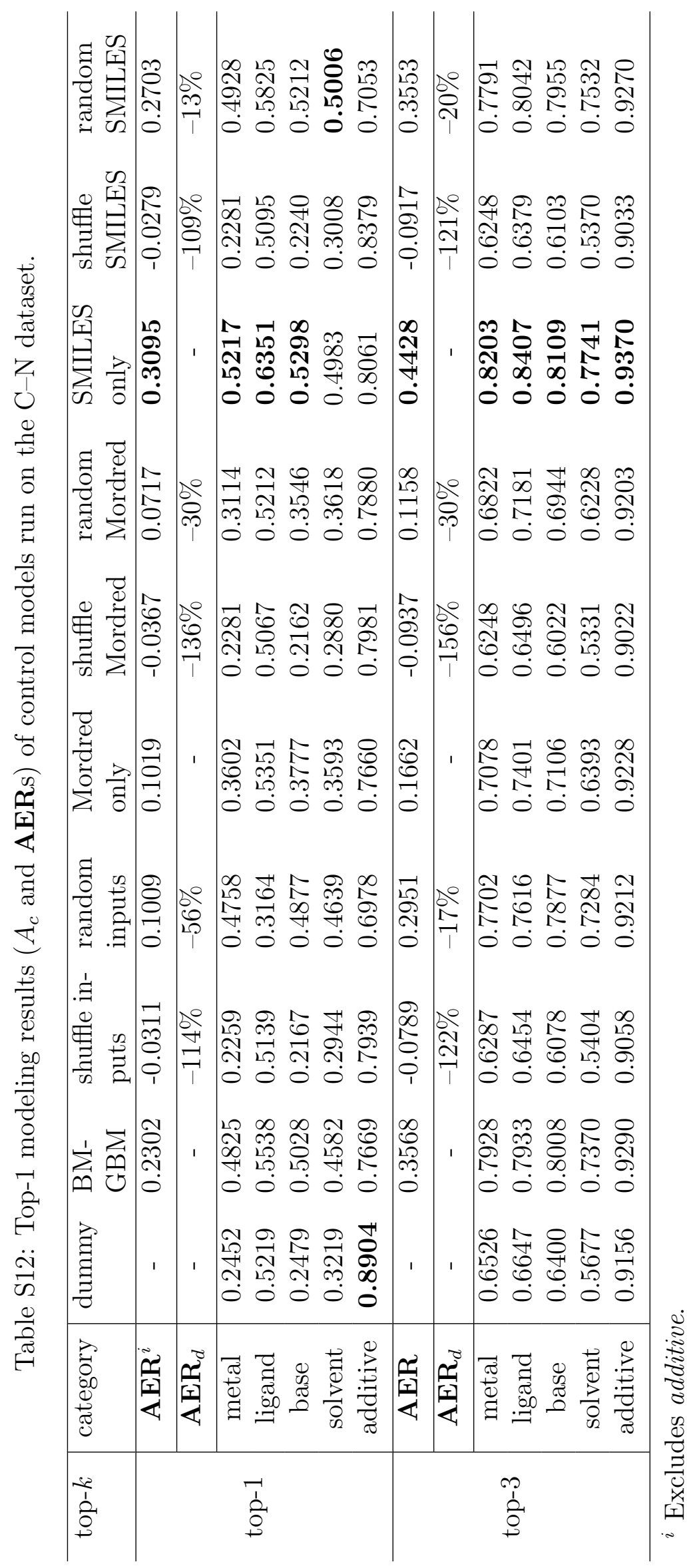




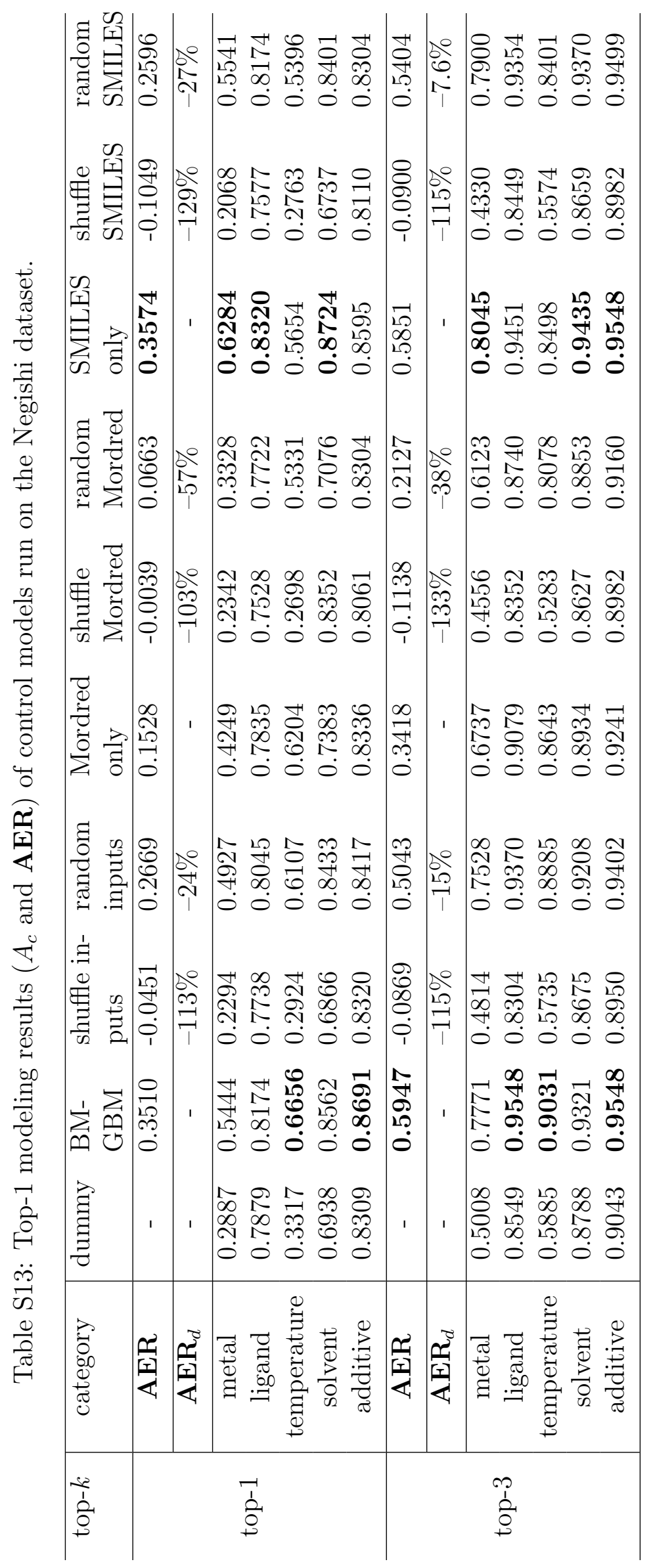




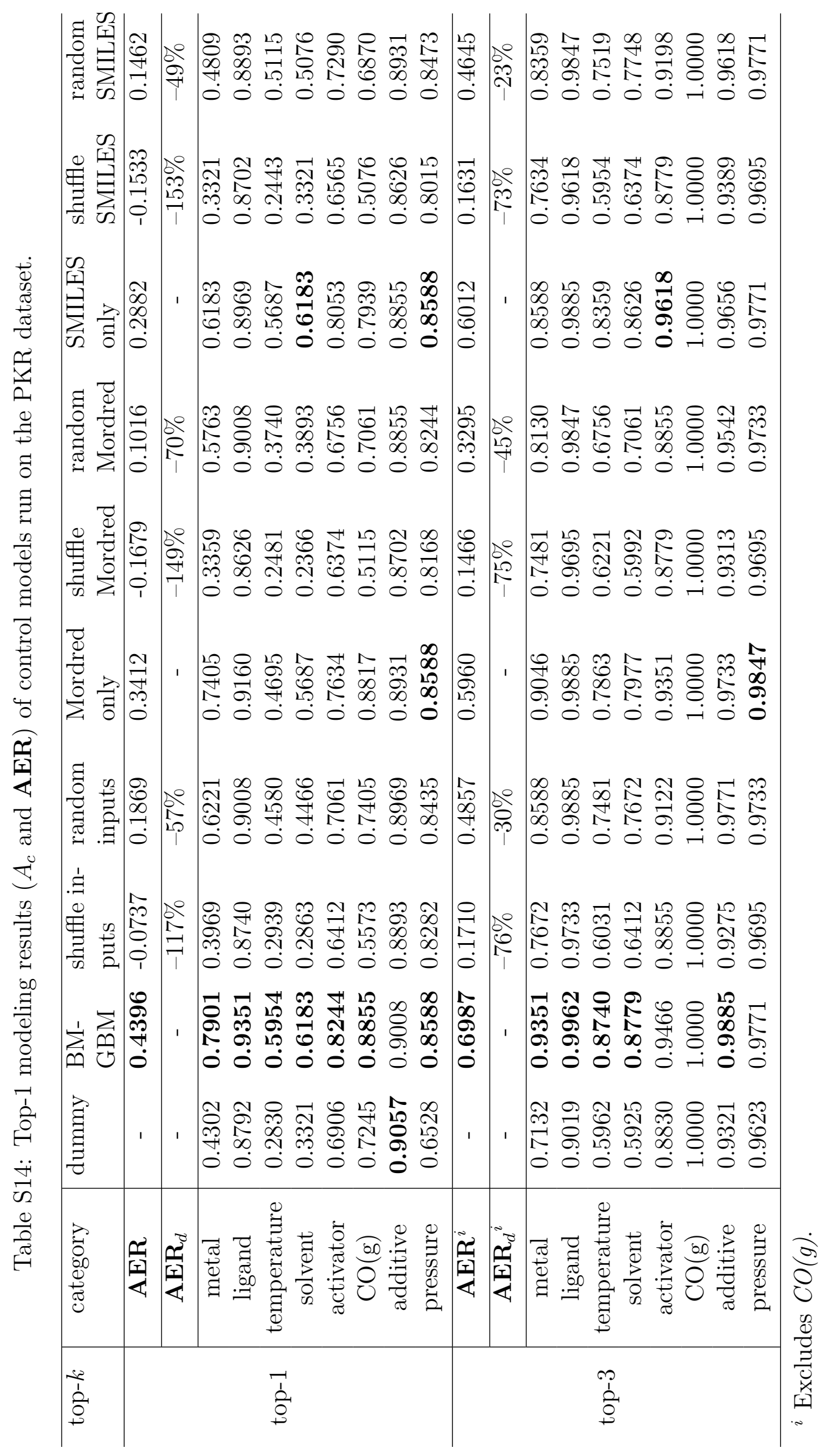




\section{S4.1 CT-GBMs}

Additional controls were conducted for the classifier trellis (CT) models where the propagated label predictions were withheld from downstream models. As above, $\mathrm{AER}_{d}$ values were recorded for these straw models relative to their featurized CT reference models. Top-1 and top-3 results for these experiments are included in Table S15 and S16, respectively. These results indicate that models heavily rely on upstream label information, and perhaps overfit to these input features.

It is interesting to note that the strongest drop in performance on holdout comes from heavily skewed categories (see $\mathrm{C}-\mathrm{N}$ ligand and additive, for example). This is perhaps sensible in that the highest frequency bin in these categories is the NULL label. As such, it appears that interdependent models for these categories base their predictions largely on whether or not a reagent is expected to be used in their category at all. This seems to overtake most structural information in the inputs and when propagated predictions are removed, the models break down. 


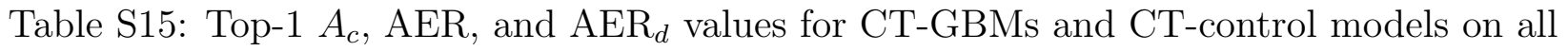
four datasets.

\begin{tabular}{|c|c|c|c|c|}
\hline dataset & category & dummy & CT-GBM & CT-holdout \\
\hline \multirow{7}{*}{ Suzuki } & $\mathbf{A E R}^{i}$ & - & 0.0922 & -2.0013 \\
\hline & $\boldsymbol{A E R _ { \boldsymbol { d } } ^ { i }}$ & - & - & $-2271 \%$ \\
\hline & metal & 0.3777 & 0.5629 & 0.3967 \\
\hline & ligand & 0.8722 & 0.8408 & 0.0302 \\
\hline & base & 0.3361 & 0.4777 & 0.3329 \\
\hline & solvent & 0.6377 & 0.6751 & 0.1143 \\
\hline & additive & 0.9511 & 0.9196 & 0.0217 \\
\hline \multirow{7}{*}{$\mathrm{C}-\mathrm{N}$} & $\mathbf{A E R}^{i}$ & - & 0.2282 & -0.2362 \\
\hline & $\boldsymbol{A E R _ { d }}{ }^{i}$ & - & - & $-204 \%$ \\
\hline & metal & 0.2452 & 0.4582 & 0.2284 \\
\hline & ligand & 0.5219 & 0.5710 & 0.0869 \\
\hline & base & 0.2479 & 0.5003 & 0.2435 \\
\hline & solvent & 0.3219 & 0.4524 & 0.3173 \\
\hline & additive & 0.8904 & 0.7031 & 0.0136 \\
\hline \multirow{7}{*}{ Negishi } & AER & - & 0.2773 & $-0.9997^{i}$ \\
\hline & $A E R_{d}$ & - & - & $-461 \%$ \\
\hline & metal & 0.2887 & 0.5218 & 0.2827 \\
\hline & ligand & 0.7879 & 0.7900 & 0.0420 \\
\hline & temperature & 0.3317 & 0.6527 & 0.0129 \\
\hline & solvent & 0.6938 & 0.8514 & 0.6947 \\
\hline & additive & 0.8309 & 0.8401 & 0.0452 \\
\hline \multirow{10}{*}{ PKR } & AER & - & 0.4010 & $-1.7313^{i}$ \\
\hline & $A E R_{d}$ & - & - & $-532 \%$ \\
\hline & metal & 0.4302 & 0.7786 & 0.2137 \\
\hline & ligand & 0.8792 & 0.9237 & 0.0496 \\
\hline & temperature & 0.2830 & 0.5649 & 0.0191 \\
\hline & solvent & 0.3321 & 0.6260 & 0.3626 \\
\hline & activator & 0.6906 & 0.8015 & 0.1412 \\
\hline & $\mathrm{CO}(\mathrm{g})$ & 0.7245 & 0.8855 & 0.4580 \\
\hline & additive & 0.9057 & 0.8893 & 0.0267 \\
\hline & pressure & 0.6528 & 0.8702 & 0.0267 \\
\hline
\end{tabular}

${ }^{i}$ Excludes additive 
Table S16: Top-3 $A_{c}$, AER, and AER $d$ values for CT-GBMs and CT-control models on all four datasets.

\begin{tabular}{|c|c|c|c|c|}
\hline dataset & category & dummy & CT-GBM & CT-holdout \\
\hline \multirow{7}{*}{ Suzuki } & $\mathbf{A E R}^{i}$ & - & 0.3774 & -4.2619 \\
\hline & $\boldsymbol{A} \boldsymbol{E} \boldsymbol{R}_{\boldsymbol{d}}^{i}$ & - & - & $-1229 \%$ \\
\hline & metal & 0.6744 & 0.8475 & 0.3981 \\
\hline & ligand & 0.9269 & 0.9606 & 0.0325 \\
\hline & base & 0.7344 & 0.8250 & 0.3350 \\
\hline & solvent & 0.8013 & 0.8577 & 0.3110 \\
\hline & additive & 0.9771 & 0.9832 & 0.0238 \\
\hline \multirow{7}{*}{$\mathrm{C}-\mathrm{N}$} & $\mathbf{A E R}^{i}$ & - & 0.3832 & -0.8693 \\
\hline & $\boldsymbol{A} \boldsymbol{E} \boldsymbol{R}_{d}^{i}$ & - & - & $-327 \%$ \\
\hline & metal & 0.6526 & 0.7772 & 0.2320 \\
\hline & ligand & 0.6647 & 0.7928 & 0.0922 \\
\hline & base & 0.6400 & 0.7916 & 0.6373 \\
\hline & solvent & 0.5677 & 0.7281 & 0.3290 \\
\hline & additive & 0.9156 & 0.9184 & 0.0148 \\
\hline \multirow{7}{*}{ Negishi } & AER & - & 0.5199 & $-0.6714^{i}$ \\
\hline & $A E R_{d}$ & - & - & $-229 \%$ \\
\hline & metal & 0.5008 & 0.7674 & 0.2924 \\
\hline & ligand & 0.8549 & 0.9321 & 0.0468 \\
\hline & temperature & 0.5885 & 0.8772 & 0.4572 \\
\hline & solvent & 0.8789 & 0.9402 & 0.8821 \\
\hline & additive & 0.9043 & 0.9354 & 0.0501 \\
\hline \multirow{10}{*}{ PKR } & $\mathbf{A E R}^{i i}$ & - & 0.6740 & $-2.6600^{i i}$ \\
\hline & $A E R_{d}^{i}$ & - & - & $-495 \%$ \\
\hline & metal & 0.7132 & 0.9313 & 0.2137 \\
\hline & ligand & 0.9019 & 0.9924 & 0.1107 \\
\hline & temperature & 0.5962 & 0.8321 & 0.4580 \\
\hline & solvent & 0.5925 & 0.8550 & 0.6756 \\
\hline & activator & 0.8830 & 0.9275 & 0.2519 \\
\hline & $\mathrm{CO}(\mathrm{g})$ & 1.0000 & 1.0000 & 1.0000 \\
\hline & additive & 0.9321 & 0.9885 & 0.0611 \\
\hline & pressure & 0.9623 & 0.9847 & 0.9389 \\
\hline
\end{tabular}

${ }^{i}$ Excludes additive

${ }^{i i}$ Excludes CO (g) 


\section{S5 Interpretability}

\section{S5.1 GBM feature importances (FIs)}

FIs were calculated and averaged over all classifiers in all four BM-GBM models and the randomized control models (random inputs). The FIs plotted over the full input space are shown below. These show uniform decay in the SMILES region, where the token vectors for the three molecules (two reactants + one product) were padded to length 100 each, and thus the frequency of character presence decays with the vector position.

The FIs from the Mordred ${ }^{\underline{S 2}}$ vector region were isolated and analyzed for their chemical significance (see main text for discussion). The top 20 FIs from this region are summarized in the tables below. Full length FI rankings and FI charts for all four models and controls are included in the code repository. 


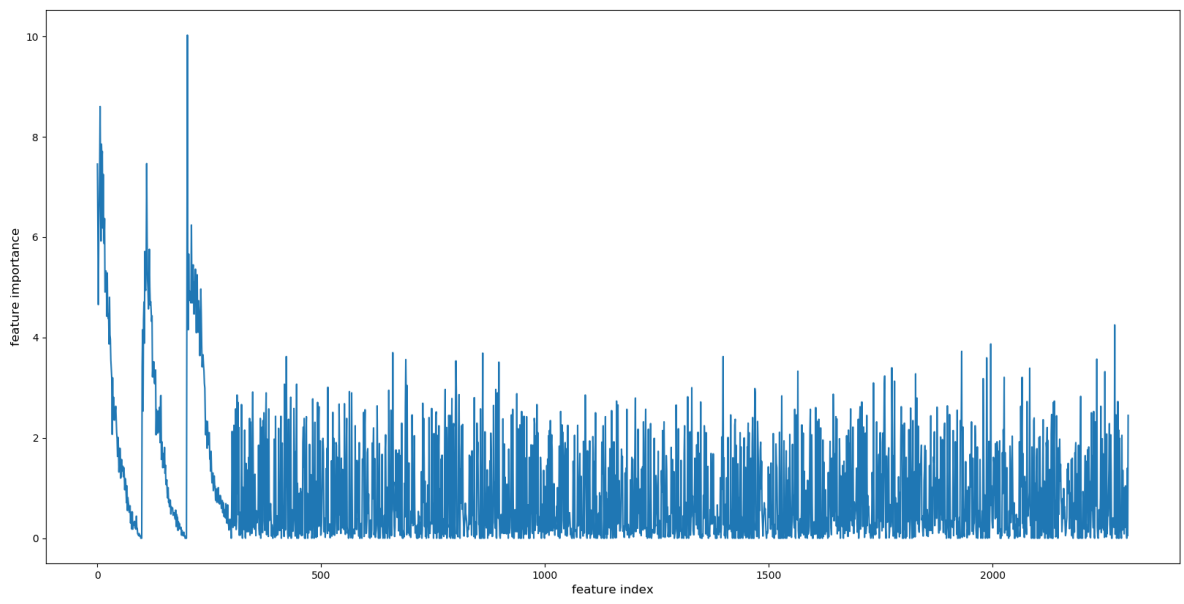

Figure S9: Relative feature importances for the full vector inputs averaged over the Suzuki BM-GBM classifiers.

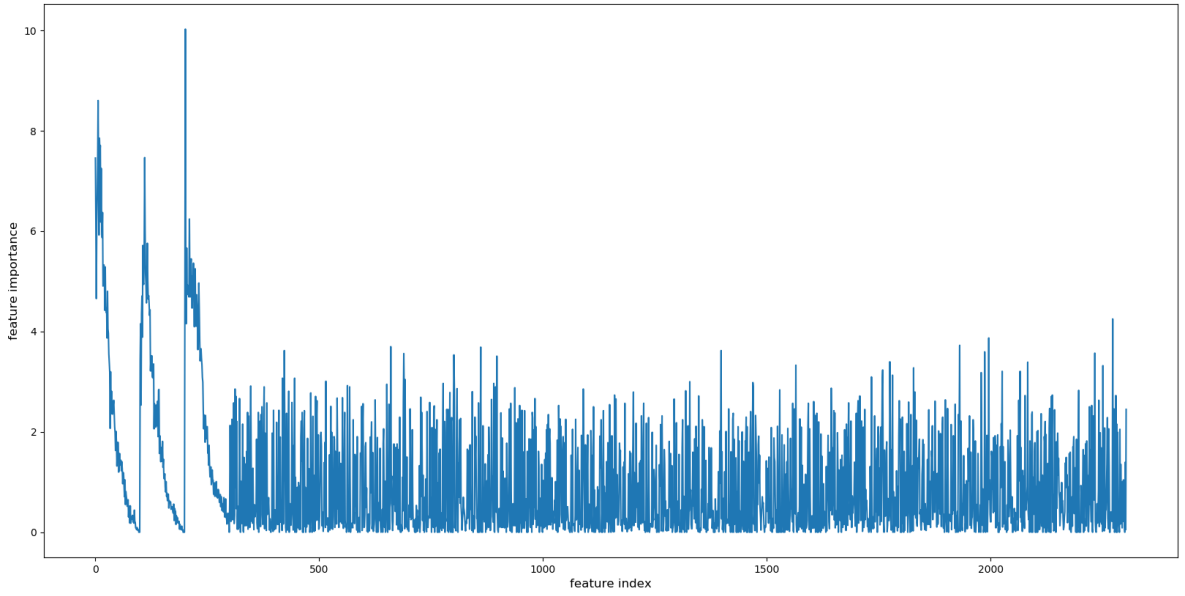

Figure S10: Relative feature importances for randomized vector inputs averaged over the Suzuki BM-GBM classifiers. 
Table S17: Top-20 Mordred descriptor FIs for Suzuki BM-GBMs with chemical explanations.

\begin{tabular}{|c|c|c|c|c|}
\hline rank & descriptor & species & FI score & description \\
\hline 1 & JGI6 & product & 4.2500 & 6 -ordered mean topological charge ${ }^{a}$ \\
\hline 2 & JGI3 & product & 3.8707 & 3-ordered mean topological charge ${ }^{a}$ \\
\hline 3 & JGI5 & product & 3.7241 & 5 -ordered mean topological charge ${ }^{a}$ \\
\hline 4 & JGI3 & reactant 1 & 3.6983 & 3 -ordered mean topological charge ${ }^{a}$ \\
\hline 5 & AATSC0p & reactant 1 & 3.6897 & $\begin{array}{l}\text { averaged and centered Moreau-Broto } \\
\text { autocorrelation of lag } 0 \text { weighted by } \\
\text { polarizability }^{b}\end{array}$ \\
\hline 6 & $\mathrm{SsOH}$ & reactant 2 & 3.6207 & sum of $\mathrm{sOH}^{c}$ \\
\hline 7 & IC1 & reactant 1 & 3.6207 & $\begin{array}{l}\text { 1-ordered neighborhood information } \\
\text { content }^{d}\end{array}$ \\
\hline 8 & JGI4 & product & 3.5948 & 4-ordered mean topological charge ${ }^{a}$ \\
\hline 9 & SdssC & product & 3.5690 & sum of $\mathrm{dss}^{e}$ \\
\hline 10 & ATSC3m & reactant 1 & 3.5603 & $\begin{array}{l}\text { centered Moreau-Broto autocorrelation of } \\
\text { lag } 3 \text { weighted by mass }{ }^{b}\end{array}$ \\
\hline 11 & EState_VSA6 & reactant 1 & 3.5345 & EState VSA Descriptor $6(<=\mathrm{x}<)^{f}$ \\
\hline 12 & SdssC & reactant 1 & 3.5086 & sum of $\mathrm{dss}^{e}$ \\
\hline 13 & JGI9 & product & 3.3966 & 9-ordered mean topological charge ${ }^{a}$ \\
\hline 14 & ATSC4i & product & 3.3879 & $\begin{array}{l}\text { centered Moreau-Broto autocorrelation of } \\
\text { lag } 4 \text { weighted by ionization potential }{ }^{b}\end{array}$ \\
\hline 15 & & reactant 2 & 3.3276 & sum of $\mathrm{dss} \mathrm{C}^{e}$ \\
\hline 16 & SlogP_VSA8 & product & 3.3190 & $\begin{array}{l}\text { MOE } \log \mathrm{P} \text { VSA Descriptor } 8(0.25<=\mathrm{x} \\
<0.30)^{g}\end{array}$ \\
\hline 17 & JGI2 & product & 3.2759 & 2-ordered mean topological charge ${ }^{a}$ \\
\hline 18 & JGI8 & product & 3.2328 & 8-ordered mean topological charge ${ }^{a}$ \\
\hline 19 & PEOE_VSA8 & product & 3.2069 & $\begin{array}{l}\text { MOE charge VSA Descriptor } 8(0.00<= \\
\mathrm{x}<0.05)^{h}\end{array}$ \\
\hline 20 & $\mathrm{SsOH}$ & product & 3.2069 & sum of $\mathrm{sOH}^{c}$ \\
\hline
\end{tabular}

${ }^{a}$-ordered mean topological charge describes sum of atom-pair charge-transfer terms up to edge-distance $n$, averaged over all atoms in a molecule. $\underline{[\mathrm{S} 9]}$

${ }^{b}$ Moreau-Broto autocorrelation of lag $n$ weighted by property $p$ describes the distribution of $p$ values over all atom pairs of edge-distance $n$.

${ }^{c}$ Sum of electrotopological state of free-alcohol oxygens. $\underline{\text { S12 IS13 }}$

${ }^{d}$ Measures graph complexity by summing local symmetry over nodes with unique neighborhoods at edge-distance 1 . S14

${ }^{e}$ Sum of electrotopological state of disubstituted $\mathrm{sp}^{2}$ carbons. $\stackrel{\text { S12[S13 }}{ }$

${ }^{f}$ Describes the sum of the van der Waals surface area (VSA) with electrotopological state in the range 1.81-2.05. $\underline{\text { S12|S14|S15 }}$

${ }^{g}$ Describes the sum of the VSA with SlogP (hybrid atomistic $\log \mathrm{P}$ ) in the range 0.25$0.30 . \underline{\mathrm{S} 12 \mathrm{~S} 16}$

${ }^{h}$ Describes the sum of the VSA with partial charge in the range 0.00-0.05. $\underline{\text { S12 }}$ 


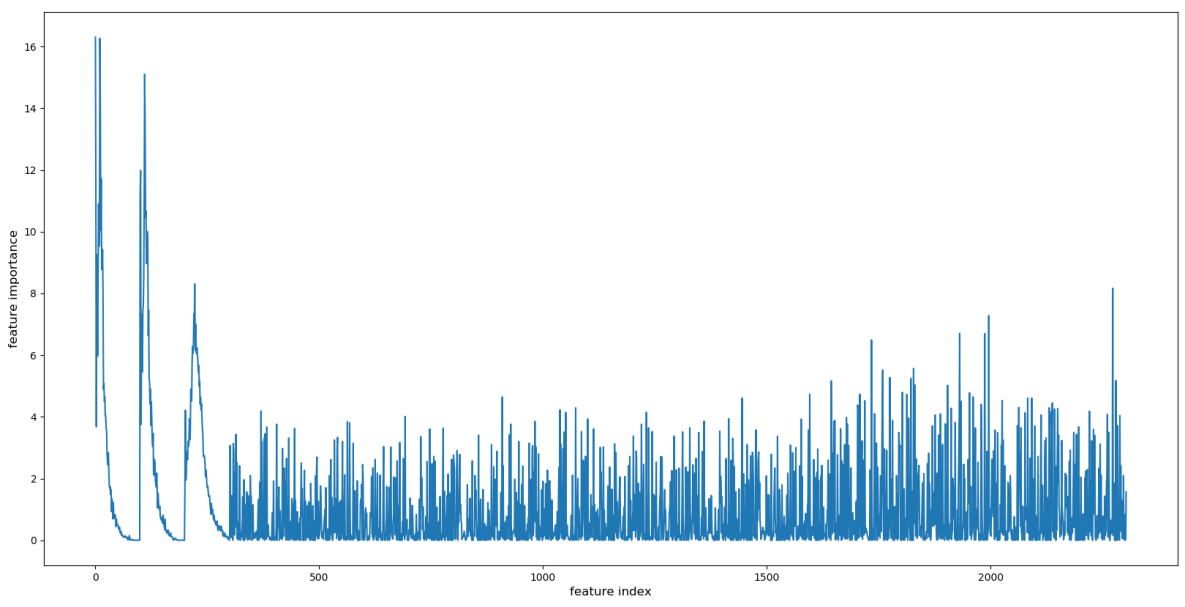

Figure S11: Relative feature importances for the full vector inputs averaged over the C-N BM-GBM classifiers.

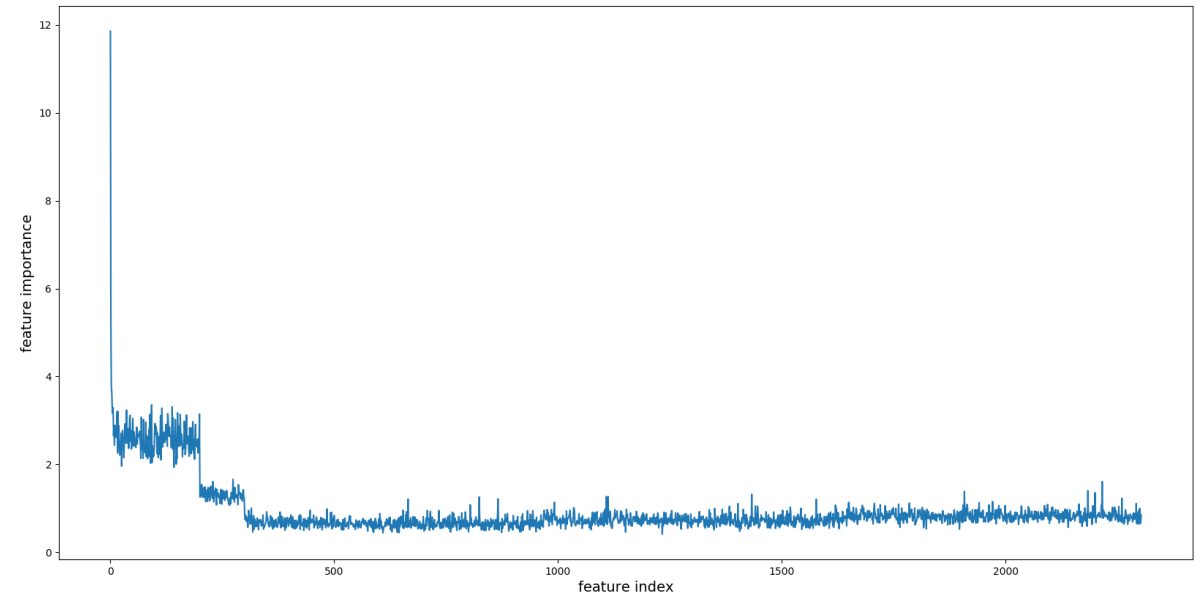

Figure S12: Relative feature importances for randomized vector inputs averaged over the C-N BM-GBM classifiers. 
Table S18: Top-20 Mordred descriptor FIs for C-N BM-GBMs with chemical explanations.

\begin{tabular}{|c|c|c|c|c|}
\hline rank & descriptor & species & FI score & description \\
\hline 1 & JGI6 & product & 8.1667 & 6-ordered mean topological charge $\mathrm{a}^{a}$ \\
\hline 2 & JGI3 & product & 7.2778 & 3 -ordered mean topological charge ${ }^{a}$ \\
\hline 3 & JGI5 & product & 6.7071 & 5-ordered mean topological charge ${ }^{a}$ \\
\hline 4 & JGI4 & product & 6.6970 & 4-ordered mean topological charge ${ }^{a}$ \\
\hline 5 & JGI7 & product & 6.4899 & 7-ordered mean topological charge ${ }^{a}$ \\
\hline 6 & JGI2 & product & 5.5707 & 2-ordered mean topological charge ${ }^{a}$ \\
\hline 7 & JGI8 & product & 5.5152 & 8-ordered mean topological charge ${ }^{a}$ \\
\hline 8 & JGI9 & product & 5.2727 & 9 -ordered mean topological charge ${ }^{a}$ \\
\hline 9 & JGI10 & product & 5.2424 & 9 -ordered mean topological charge ${ }^{a}$ \\
\hline 10 & ATSC8d & product & 5.1717 & $\begin{array}{l}\text { centered Moreau-Broto autocorrelation of } \\
\text { lag } 8 \text { weighted by sigma electrons }{ }^{b}\end{array}$ \\
\hline 11 & CIC3 & product & 5.1667 & $\begin{array}{l}\text { 3-ordered complementary information } \\
\text { content }^{c}\end{array}$ \\
\hline 12 & ATSC2dv & product & 5.0303 & $\begin{array}{l}\text { centered Moreau-Broto autocorrelation of } \\
\text { lag } 2 \text { weighted by valence electrons }{ }^{b}\end{array}$ \\
\hline 13 & ATSC5i & product & 5.0202 & $\begin{array}{l}\text { centered Moreau-Broto autocorrelation of } \\
\text { lag } 5 \text { weighted by ionization potential }\end{array}$ \\
\hline 14 & ATSC7i & product & 4.7929 & $\begin{array}{l}\text { centered Moreau-Broto autocorrelation of } \\
\text { lag } 5 \text { weighted by ionization potential }{ }^{b}\end{array}$ \\
\hline 15 & MIC1 & product & 4.7778 & $\begin{array}{l}\text { 1-ordered modified information content } \\
\text { weighted by mass }\end{array}$ \\
\hline 16 & ATSC5p & reactant 2 & 4.7323 & $\begin{array}{l}\text { centered Moreau-Broto autocorrelation of } \\
\text { lag } 5 \text { weighted by polarizability }\end{array}$ \\
\hline 17 & EState_VSA7 & product & 4.7323 & $\begin{array}{l}\text { EState VSA Descriptor } 7(1.81<=\mathrm{x}< \\
2.05)^{d}\end{array}$ \\
\hline 18 & CIC4 & product & 4.7273 & $\begin{array}{l}\text { 4-ordered complementary information } \\
\text { content }^{c}\end{array}$ \\
\hline 19 & ATSC4dv & product & 4.6465 & $\begin{array}{l}\text { centered Moreau-Broto autocorrelation of } \\
\text { lag } 4 \text { weighted by valence electrons }{ }^{b}\end{array}$ \\
\hline 20 & ATSC5se & reactant 1 & 4.6414 & $\begin{array}{l}\text { centered Moreau-Broto autocorrela- } \\
\text { tion of lag } 5 \text { weighted by Sanderson } \\
\text { electronegativity }{ }^{b}\end{array}$ \\
\hline
\end{tabular}

${ }^{a} n$-ordered mean topological charge describes sum of atom-pair charge-transfer terms up to edge-distance $n$, averaged over all atoms in a molecule. $\$$

${ }^{b}$ Moreau-Broto autocorrelation of lag $n$ weighted by property $p$ describes the distribution of $p$ values over all atom pairs of edge-distance $n$. S10|S11

${ }^{c}$ Difference between actual and maximum possible graph complexity as sum of local symmetry over nodes with unique neighborhoods at edge-distance $3 . \underline{\$ 14}$

${ }^{d}$ Describes the sum of the van der Waals surface area (VSA) with electrotopological state in the range 1.81-2.05. $1212[\mathrm{~S} 14 \mid \mathrm{S} 15]$ 


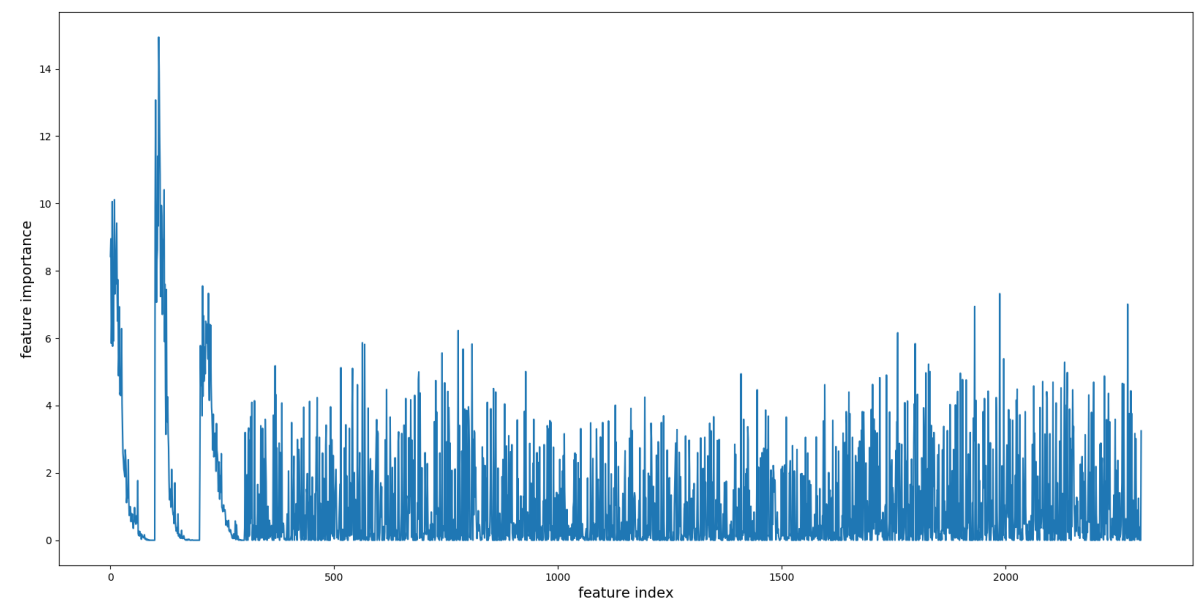

Figure S13: Relative feature importances for the full vector inputs averaged over the Negishi BM-GBM classifiers.

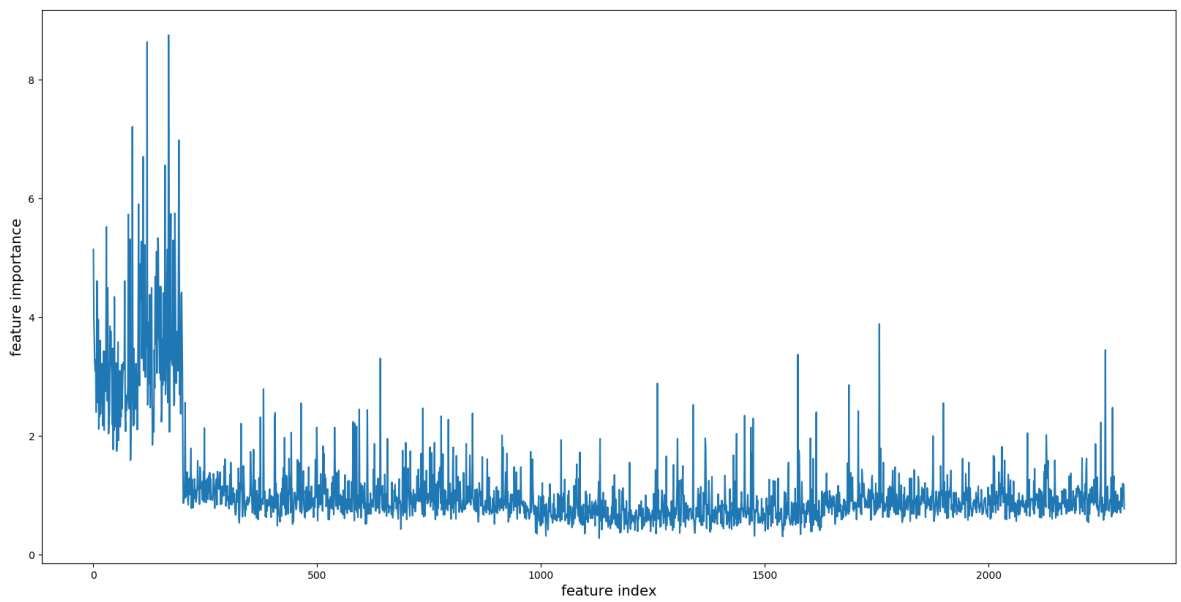

Figure S14: Relative feature importances for randomized vector inputs averaged over the Negishi BM-GBM classifiers. 
Table S19: Top-20 Mordred descriptor FIs for Negishi BM-GBMs with chemical explanations.

\begin{tabular}{|c|c|c|c|c|}
\hline rank & descriptor & species & FI score & description \\
\hline 1 & JGI4 & product & 7.3238 & 4-ordered mean topological charge $^{a}$ \\
\hline 2 & JGI6 & product & 7.0095 & 6 -ordered mean topological charge ${ }^{a}$ \\
\hline 3 & JGI5 & product & 6.9429 & 5 -ordered mean topological charge ${ }^{a}$ \\
\hline 4 & ATSC4p & reactant 1 & 6.2286 & $\begin{array}{l}\text { centered Moreau-Broto autocorrelation of } \\
\text { lag } 4 \text { weighted by polarizability }\end{array}$ \\
\hline 5 & JGI8 & product & 6.1619 & 8-ordered mean topological charge ${ }^{a}$ \\
\hline 6 & AATSC0i & reactant 1 & 5.8667 & $\begin{array}{l}\text { averaged and centered Moreau-Broto auto- } \\
\text { correlation of lag } 0 \text { weighted by ionization } \\
\text { potential }^{b}\end{array}$ \\
\hline 7 & SaasC & product & 5.8381 & sum of aas $\mathrm{C}^{c}$ \\
\hline 8 & $\mathrm{SsBr}$ & reactant 1 & 5.8286 & sum of $\mathrm{sBr}^{d}$ \\
\hline 9 & ATSC5i & reactant 1 & 5.8190 & $\begin{array}{l}\text { centered Moreau-Broto autocorrelation of } \\
\text { lag } 5 \text { weighted by ionization potential }{ }^{b}\end{array}$ \\
\hline 10 & ATSC6se & reactant 1 & 5.6762 & $\begin{array}{l}\text { centered Moreau-Broto autocorrela- } \\
\text { tion of lag } 6 \text { weighted by Sanderson } \\
\text { electronegativity }^{b}\end{array}$ \\
\hline 11 & ATSC1se & reactant 1 & 5.5619 & $\begin{array}{l}\text { centered Moreau-Broto autocorrela- } \\
\text { tion of lag } 1 \text { weighted by Sanderson } \\
\text { electronegativity }^{b}\end{array}$ \\
\hline 12 & JGI3 & product & 5.3905 & 3 -ordered mean topological charge ${ }^{a}$ \\
\hline 13 & ATSC4d & product & 5.2857 & $\begin{array}{l}\text { centered Moreau-Broto autocorrelation of } \\
\text { lag } 4 \text { weighted by sigma electrons }{ }^{b}\end{array}$ \\
\hline 14 & JGI2 & product & 5.2286 & 2-ordered mean topological charge ${ }^{a}$ \\
\hline 15 & $\mathrm{ZMIC} 2$ & reactant 1 & 5.1810 & $\begin{array}{l}\text { 2-ordered Z-modified information content } \\
\text { weighted by atomic number }\end{array}$ \\
\hline 16 & $\mathrm{IC} 0$ & reactant 1 & 5.1238 & 0 -ordered information content ${ }^{e}$ \\
\hline 17 & bpol & reactant 1 & 5.1048 & bond polarizability ${ }^{f}$ \\
\hline 18 & ATSC2dv & product & 5.0095 & $\begin{array}{l}\text { centered Moreau-Broto autocorrelation of } \\
\text { lag } 2 \text { weighted by valence electrons }{ }^{b}\end{array}$ \\
\hline 19 & ATSC5p & reactant 1 & 5.0095 & $\begin{array}{l}\text { centered Moreau-Broto autocorrelation of } \\
\text { lag } 5 \text { weighted by polarizability }{ }^{b}\end{array}$ \\
\hline 20 & ATSC3m & reactant 1 & 5.0000 & $\begin{array}{l}\text { centered Moreau-Broto autocorrelation of } \\
\text { lag } 3 \text { weighted by mass }{ }^{b}\end{array}$ \\
\hline
\end{tabular}

${ }^{a} n$-ordered mean topological charge describes sum of atom-pair charge-transfer terms up to edge-distance $n$, averaged over all atoms in a molecule. $\underline{\text { S9 }}$

${ }^{b}$ Moreau-Broto autocorrelation of lag $n$ weighted by property $p$ describes the distribution of $p$ values over all atom pairs of edge-distance $n . \underline{\text { S10|S11 }}$

${ }^{c}$ Sum of electrotopological state of substituted aromatic carbons. $\mathbf{S 1 2}$

${ }^{d}$ Sum of electrotopological state of organobromides. $\stackrel{\text { S12 }}{ }$

e Measures graph complexity by summing local symmetry over nodes with unique neighborhoods at edge-distance 2. S14

${ }^{f}$ Sum of absolute value of polarizability differences between bound atom pairs. $\frac{\text { S2 }}{\text {. }}$ 


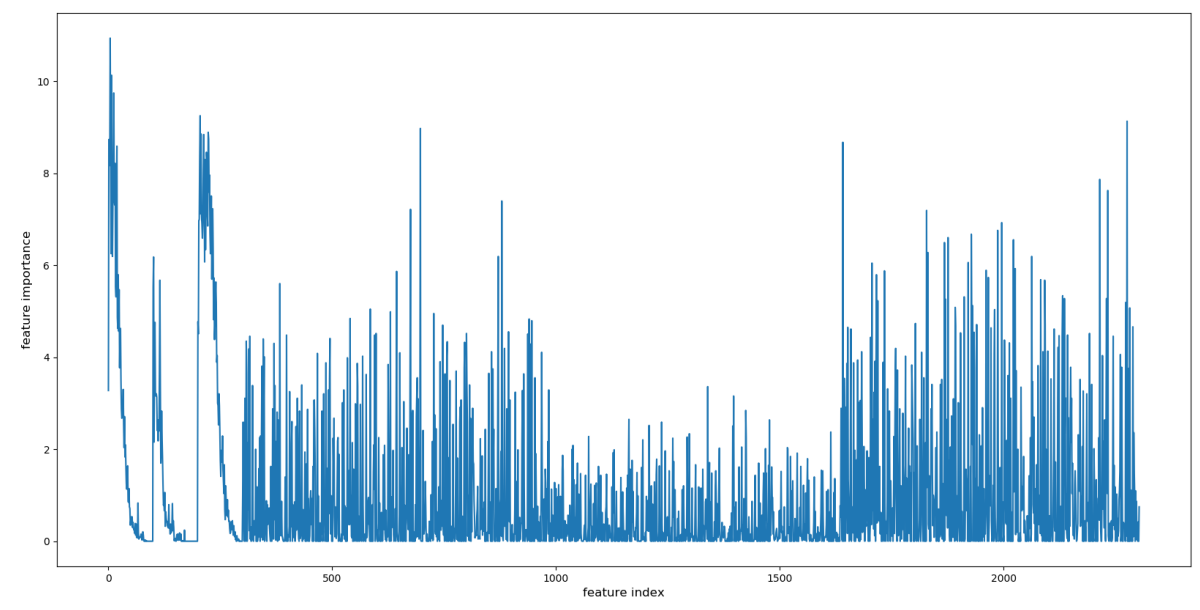

Figure S15: Relative feature importances for the full vector inputs averaged over the PKR BM-GBM classifiers.

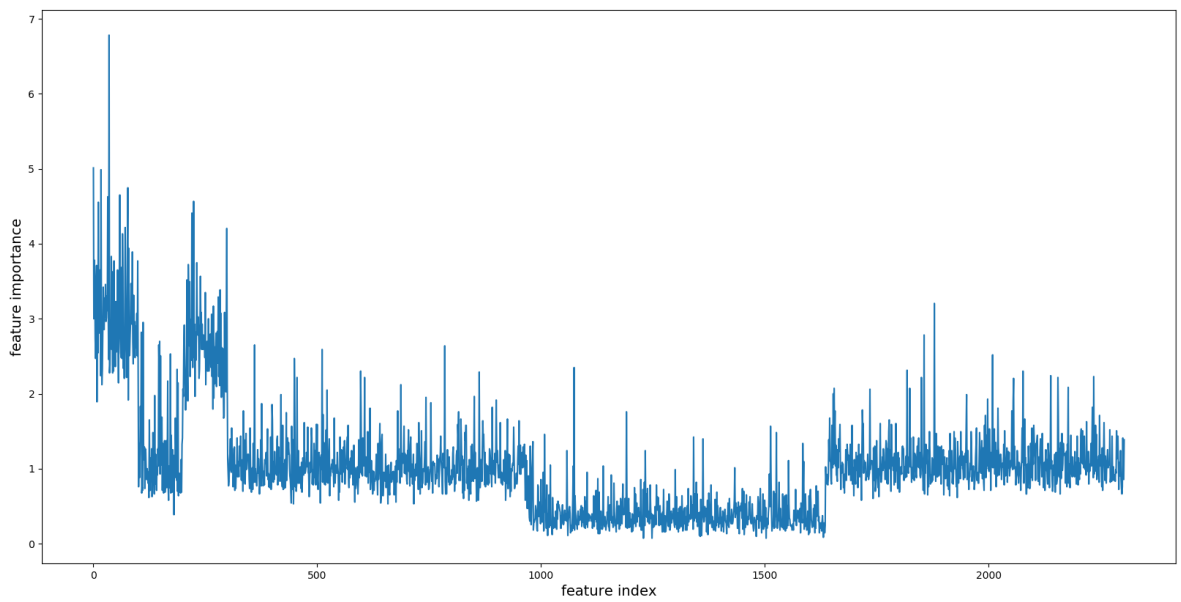

Figure S16: Relative feature importances for randomized vector inputs averaged over the PKR BM-GBM classifiers. 
Table S20: Top-20 Mordred descriptor FIs for PKR BM-GBMs with chemical explanations.

\begin{tabular}{|c|c|c|c|c|}
\hline rank & descriptor & species & FI score & description \\
\hline 1 & $\mathrm{SsssCH}$ & product & 9.1325 & sum of $\mathrm{sssCH}^{a}$ \\
\hline 2 & SddC & reactant 1 & 8.9759 & sum of $\mathrm{ddC}^{a}$ \\
\hline 3 & EState_VSA3 & product & 8.6747 & $\begin{array}{l}\text { EState VSA Descriptor } 3(0.29<=\mathrm{x}< \\
0.72)^{b}\end{array}$ \\
\hline 4 & $\mathrm{SdsCH}$ & product & 7.8675 & sum of $\mathrm{dsCH}^{a}$ \\
\hline 5 & $\mathrm{SdssC}$ & product & 7.6265 & sum of $\mathrm{dssC}^{a}$ \\
\hline 6 & $\mathrm{SdsCH}$ & reactant 1 & 7.3976 & sum of $\mathrm{dsCH}^{a}$ \\
\hline 7 & StsC & reactant 1 & 7.2169 & sum of $\mathrm{tsC}^{a}$ \\
\hline 8 & JGI2 & product & 7.1928 & 2-ordered mean topological charge ${ }^{c}$ \\
\hline 9 & JGI3 & product & 6.9277 & 3-ordered mean topological charge ${ }^{c}$ \\
\hline 10 & JGI4 & product & 6.7590 & 4-ordered mean topological charge $^{c}$ \\
\hline 11 & Xch-6dv & product & 6.6747 & $\begin{array}{l}\text { 6-ordered Chi chain weighted by valence } \\
\text { electrons }^{d}\end{array}$ \\
\hline 12 & ATSC3d & product & 6.6024 & $\begin{array}{l}\text { centered Moreau-Broto autocorrelation of } \\
\text { lag } 3 \text { weighted by sigma electrons }{ }^{e}\end{array}$ \\
\hline 13 & Xch-6d & product & 6.5542 & $\begin{array}{l}\text { 6-ordered Chi chain weighted by sigma } \\
\text { electrons }^{d}\end{array}$ \\
\hline 14 & Xch-5dv & product & 6.4940 & $\begin{array}{l}\text { 5-ordered Chi chain weighted by valence } \\
\text { electrons }^{d}\end{array}$ \\
\hline 15 & ATSC2dv & product & 6.2771 & $\begin{array}{l}\text { centered Moreau-Broto autocorrelation of } \\
\text { lag } 2 \text { weighted by valence electrons }\end{array}$ \\
\hline 16 & $\mathrm{SdCH} 2$ & reactant 1 & 6.1928 & sum of $\mathrm{dCH} 2^{a}$ \\
\hline 17 & ATSC3dv & product & 6.1928 & $\begin{array}{l}\text { centered Moreau-Broto autocorrelation of } \\
\text { lag } 3 \text { weighted by valence electrons }{ }^{e}\end{array}$ \\
\hline 18 & PEOE_VSA7 & product & 6.0602 & $\begin{array}{l}\text { MOE charge VSA Descriptor } 7(-0.05<= \\
\mathrm{x}<0.00)^{f}\end{array}$ \\
\hline 19 & ATSC4v & product & 6.0482 & $\begin{array}{l}\text { centered Moreau-Broto autocorrelation of } \\
\text { lag } 4 \text { weighted by van der Waals volume }\end{array}$ \\
\hline 20 & PEOE_VSA8 & product & 5.9277 & $\begin{array}{l}\text { MOE charge VSA Descriptor } 8(0.00<= \\
\mathrm{x}<0.05)^{f}\end{array}$ \\
\hline
\end{tabular}

${ }^{a}$ Sum of electrotopological state of: $\mathrm{sssCH}=$ tertiary aliphatic carbons; $\mathrm{ddC}=$ central allenic carbons; dsCH = monosubstituted $\mathrm{sp}^{2}$ carbons; dssC $=$ disubstituted $\mathrm{sp}^{2}$ carbons; tsC = internal alkyne carbons; dCH2 = terminal alkene carbons..$\underline{\mathrm{S} 12}$

${ }^{b}$ Describes the fraction of the van der Waals surface area (VSA) with electrotopological state in the range listed. 12 |S14|S15

${ }^{c} n$-ordered mean topological charge describes sum of atom-pair charge-transfer terms up to edge-distance $n$, averaged over all atoms in a molecule.. $\mathbf{S 9}$

${ }^{d}$ Sum of the products of connectivity degrees of atoms in edge-distance of $n$-order weighted by property $p . \underline{\text { S14 }}$

${ }^{e}$ Moreau-Broto autocorrelation of lag $n$ weighted by property $p$ describes the distribution of $p$ values over all atom pairs of edge-distance $n$.

${ }^{f}$ Sum of the VSA with partial charge in the range specified. $\mathbf{S 1 2}$ 


\section{S5.2 AR-GCN attention visualizations}

Four random reactions were chosen from each dataset for AR-GCN attention visualization (see main text for explanation). The ground truth category labels and AR-GCN predictions are included in each example.

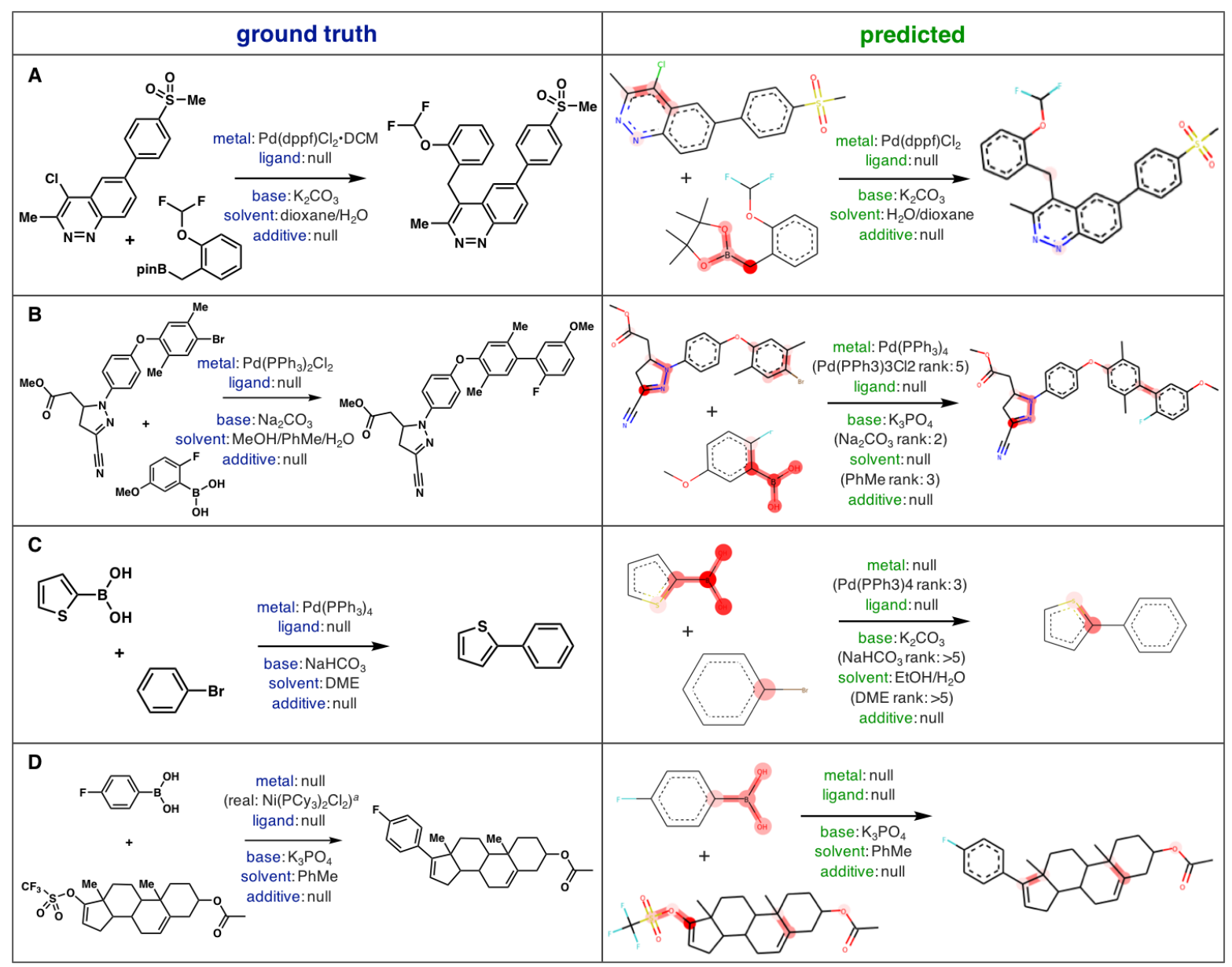

${ }^{a}$ Ground truth reagent below threshold dictionary frequency; read as null.

Figure S17: Attention visualizations for randomly chosen Suzuki couplings. 


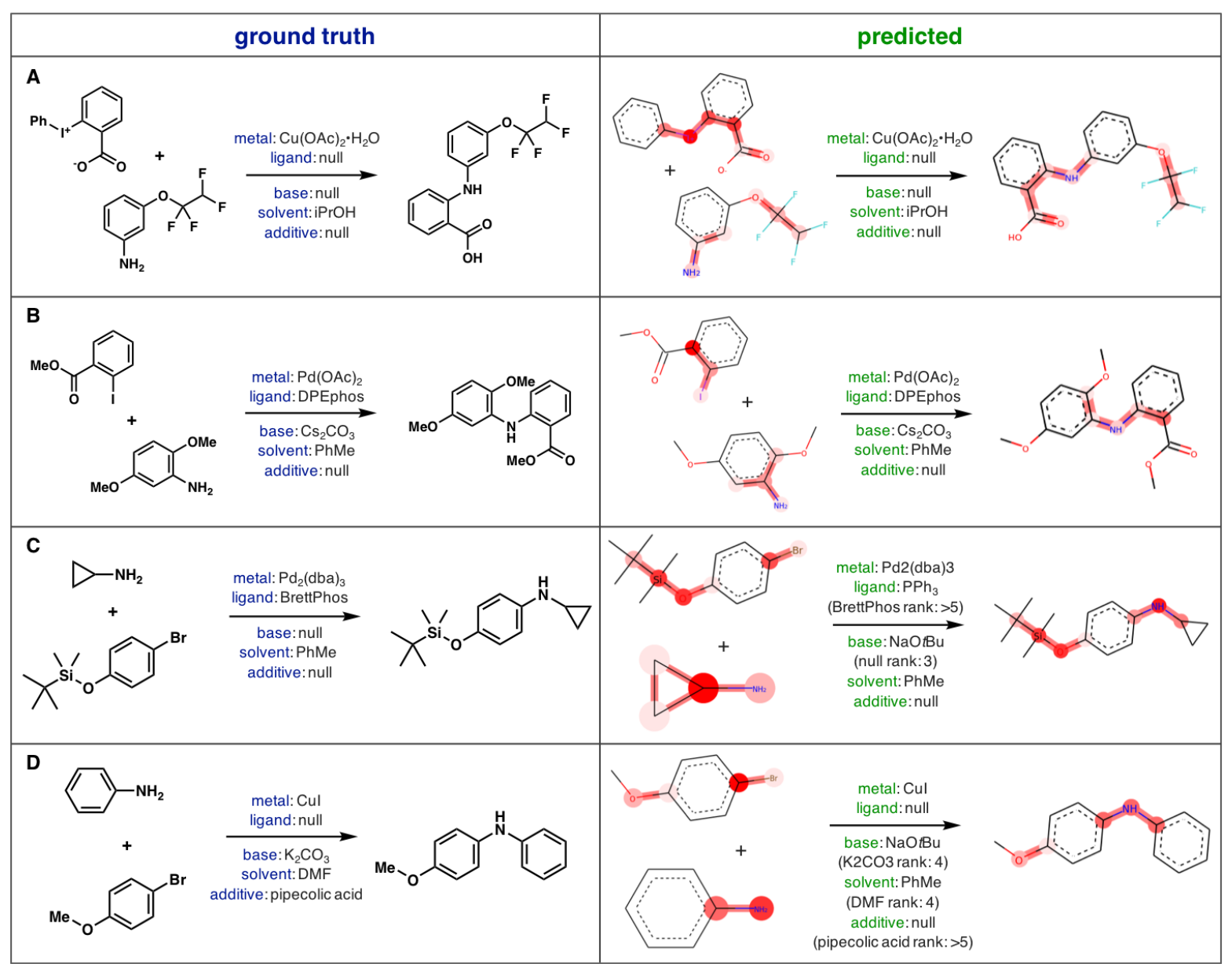

Figure S18: Attention visualizations for randomly chosen $\mathrm{C}-\mathrm{N}$ couplings. 


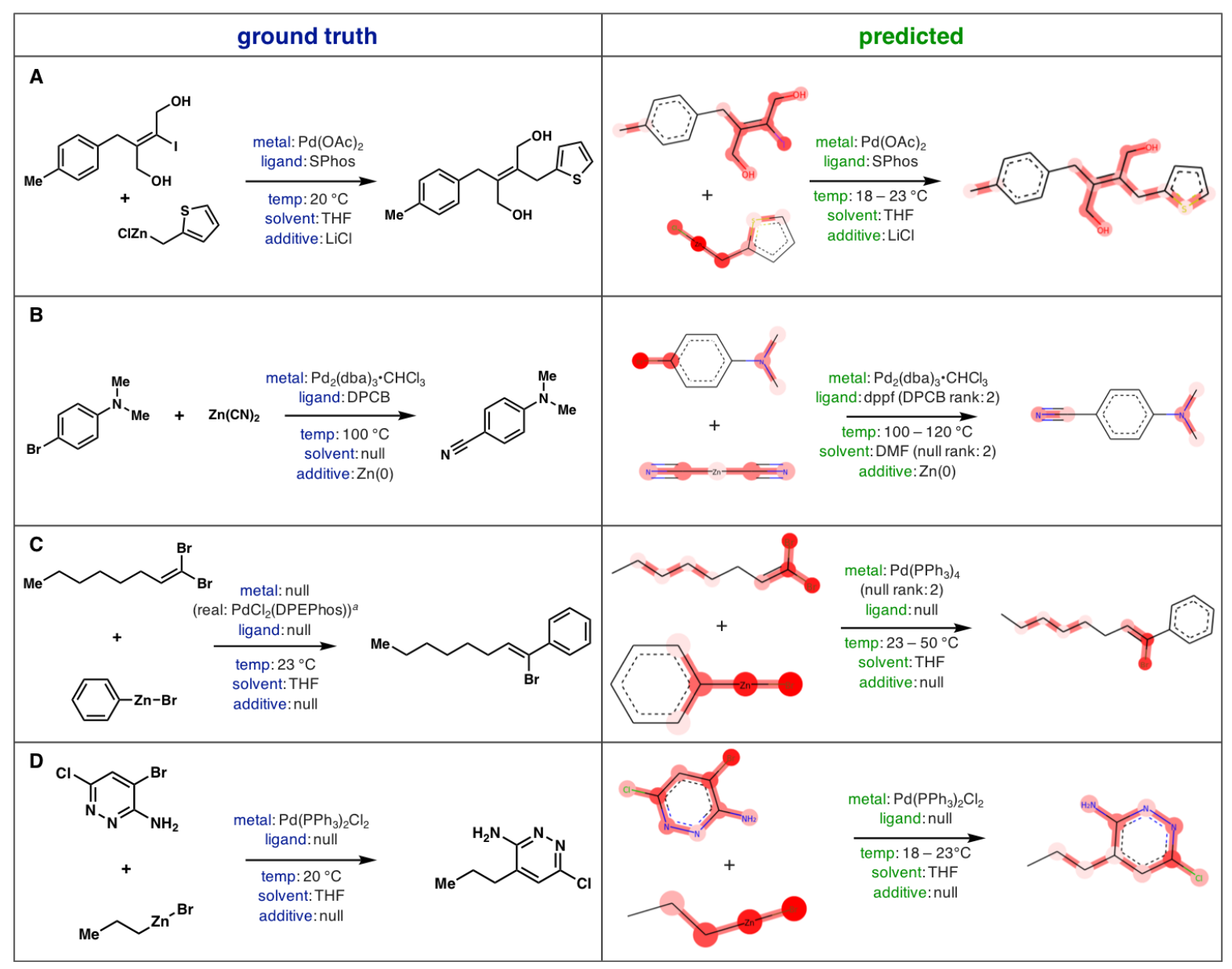

${ }^{a}$ Ground truth reagent below threshold dictionary frequency; read as null.

Figure S19: Attention visualizations for randomly chosen Negishi couplings. 


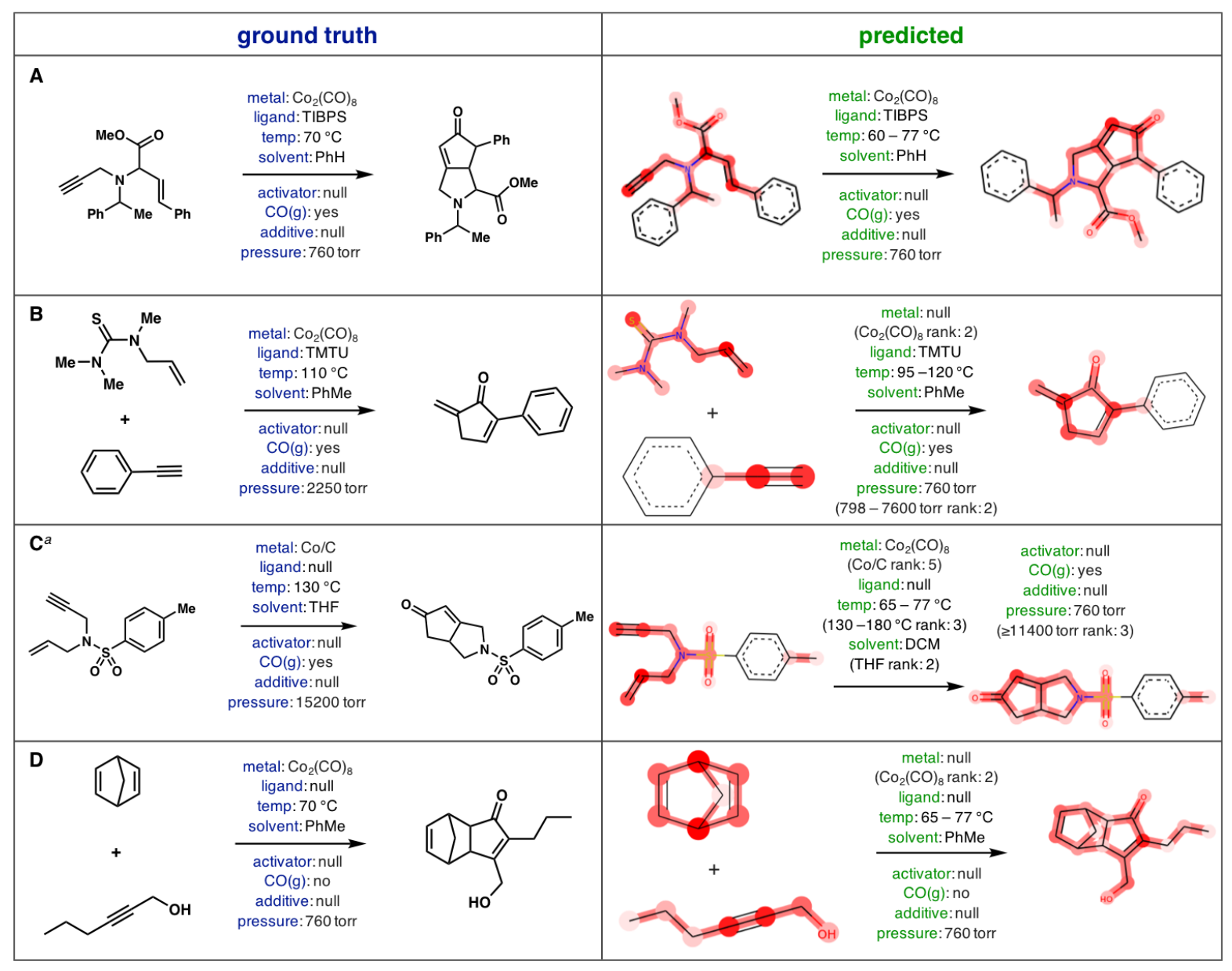

Figure S20: Attention visualizations for randomly chosen PKRs. 


\section{References}

(S1) Ryou*, S.; Maser*, M. R.; Cui*, A. Y.; DeLano, T. J.; Yue, Y.; Reisman, S. E. Graph Neural Networks for the Prediction of Substrate-Specific Organic Reaction Conditions. arXiv:2007.04275 [cs, LG] 2020,

(S2) Moriwaki, H.; Tian, Y.-S.; Kawashita, N.; Takagi, T. Mordred: a molecular descriptor calculator. Journal of Cheminformatics 2018, 10, 4.

(S3) Ke, G.; Meng, Q.; Finley, T.; Wang, T.; Chen, W.; Ma, W.; Ye, Q.; Liu, T.-Y. In Advances in Neural Information Processing Systems 30; Guyon, I., Luxburg, U. V., Bengio, S., Wallach, H., Fergus, R., Vishwanathan, S., Garnett, R., Eds.; Curran Associates, Inc., 2017; pp 3146-3154.

(S4) Pedregosa, F.; Varoquaux, G.; Gramfort, A.; Michel, V.; Thirion, B.; Grisel, O.; Blondel, M.; Prettenhofer, P.; Weiss, R.; Dubourg, V.; Vanderplas, J.; Passos, A.; Cournapeau, D. Scikit-learn: Machine Learning in Python. Journal of Machine Learning Research 2011, 12, 2825-2830.

(S5) Read, J.; Martino, L.; Olmos, P.; Luengo, D. Scalable Multi-Output Label Prediction: From Classifier Chains to Classifier Trellises. Pattern Recognition 2015, 48, 2096-2109.

(S6) Tokui, S.; Oono, K.; Hido, S.; Clayton, J. Chainer: a Next-Generation Open Source Framework for Deep Learning. 2015,

(S7) Chuang, K. V.; Keiser, M. J. Adversarial Controls for Scientific Machine Learning. ACS Chemical Biology 2018, 13, 2819-2821.

(S8) Chuang, K. V.; Keiser, M. J. Comment on "Predicting reaction performance in C-N cross-coupling using machine learning". Science 2018, 362, eaat8603.

(S9) Galvez, J.; Garcia, R.; Salabert, M. T.; Soler, R. Charge Indexes. New Topological Descriptors. Journal of Chemical Information and Modeling 1994, 34, 520-525. 
(S10) Moreau, G.; Broto, P. The Autocorrelation of a Topological Structure: A New Molecular Descriptor. New Journal of Chemistry 1980, 4, 359-360.

(S11) Hollas, B. An Analysis of the Autocorrelation Descriptor for Molecules. Journal of Mathematical Chemistry 2003, 33, 91-101.

(S12) Hall, L. H.; Mohney, B.; Kier, L. B. The electrotopological state: structure information at the atomic level for molecular graphs. Journal of Chemical Information and Modeling 1991, 31, 76-82.

(S13) Hall, L. H.; Kier, L. B. Electrotopological State Indices for Atom Types: A Novel Combination of Electronic, Topological, and Valence State Information. Journal of Chemical Information and Modeling 1995, 35, 1039-1045.

(S14) Todeschini, R.; Consonni, V. Molecular Descriptors for Chemoinformatics: Volume I: Alphabetical Listing / Volume II: Appendices, References, 1st ed.; Methods and Principles in Medicinal Chemistry; Wiley, 2009; Vol. 41.

(S15) Labute, P. A widely applicable set of descriptors. Journal of Molecular Graphics and Modelling 2000, 18, 464-477.

(S16) Wildman, S. A.; Crippen, G. M. Prediction of Physicochemical Parameters by Atomic Contributions. Journal of Chemical Information and Computer Sciences 1999, 39, $868-873$. 
